\title{
Mechanical Energy Expenditure while Maintaining Postural Stability in Shipboard Motion Environments
}

$$
\text { by }
$$

Gurwinder Kaur, B.Tech.

A thesis submitted to the Faculty of Graduate and Postdoctoral Affairs in partial fulfillment of the requirements for the degree of

Master of Applied Science

in

Biomedical Engineering

Carleton University

Ottawa, Ontario

(C) 2013

Gurwinder Kaur, B.Tech. 
Title page was reformatted to include necessary information. No information was lost. 


\title{
Mechanical Energy Expenditure while Maintaining Postural Stability in Shipboard Motion Environments
}

\author{
by
}

\author{
Gurwinder Kaur, B.Tech.
}

\author{
A Thesis submitted to \\ the Faculty of Graduate Studies and Postdoctoral Affairs \\ in partial fulfilment of \\ the requirements for the degree of \\ Master of Applied Science \\ in
}

Mechanical and Aerospace Engineering

Carleton University

Ottawa, Ontario, Canarla

January 2013

Copyright (C)

2013 - Gurwinder Kaur, B.Tech. 
Library and Archives

Canada

Published Heritage

Branch

395 Wellington Street

Ottawa ON K1A ON4

Canada
Bibliothèque et

Archives Canada

Direction du

Patrimoine de l'édition

395 , rue Wellington

Ottawa ON K1A ON4

Canada
Your file Votre référence

ISBN: 978-0-494-94266-6

Our file Notre référence

ISBN: $978-0-494-94266-6$
NOTICE:

The author has granted a nonexclusive license allowing Library and Archives Canada to reproduce, publish, archive, preserve, conserve, communicate to the public by telecommunication or on the Internet, loan, distrbute and sell theses worldwide, for commercial or noncommercial purposes, in microform, paper, electronic and/or any other formats.

The author retains copyright ownership and moral rights in this thesis. Neither the thesis nor substantial extracts from it may be printed or otherwise reproduced without the author's permission.
AVIS:

L'auteur a accordé une licence non exclusive permettant à la Bibliothèque et Archives Canada de reproduire, publier, archiver, sauvegarder, conserver, transmettre au public par télécommunication ou par l'Internet, prêter, distribuer et vendre des thèses partout dans le monde, à des fins commerciales ou autres, sur support microforme, papier, électronique et/ou autres formats.

L'auteur conserve la propriété du droit d'auteur et des droits moraux qui protege cette thèse. $\mathrm{Ni}$ la thèse ni des extraits substantiels de celle-ci ne doivent être imprimés ou autrement reproduits sans son autorisation.
In compliance with the Canadian Privacy Act some supporting forms may have been removed from this thesis.

While these forms may be included in the document page count, their removal does not represent any loss of content from the thesis.
Conformément à la loi canadienne sur la protection de la vie privée, quelques formulaires secondaires ont été enlevés de cette thèse.

Bien que ces formulaires aient inclus dans la pagination, il n'y aura aucun contenu manquant. 


\section{Abstract}

The aim of this thesis was to examine the mechanical work performed by different joints in the human body and correlate it with the metabolic energy. The motivation behind this objective was to understand human performance at sea during long maritime activities. Long-duration ship activities aggravate the chances of various motion disorders including motion-induced fatigue, motion sickness, sopite syndrome, and nausea. These disorders have been considered as major biodynamic barriers that reduce efficiency of crewmembers and ship operators during navigational tasks. Therefore the methodology of this research included implementing a mathematical model of the human body to calculate the mechanical work expended while maintaining balance. This will aid in understanding the performance of humans during such tasks and also help in formulating strategies to improve the efficiency of human performance. Experimental data from human subjects were collected on a ship motion simulator under twelve different deck motion conditions associated with four sea states and three ship headings relative to the principal wave direction for a typical frigate. Data were collected using a motion capture system, foot pressure sensors, a load cell, and a metabolic analyzer. The mechanical work performed by the human body and different body joints were calculated through the developed ninety-six degree of freedom mathematical model. The variation of metabolic levels with sea severity was investigated and a mechanical work-metabolism correlation was performed. Direct comparison of mechanical work with metabolic energy was done. Also, the variation 
of ground reaction axial force and other biological factors were calculated. The results signified that metabolic levels increase as the sea severity increases. Also, the workenergy variables were highly correlated which indicated that muscle contraction and ATP utilization increase for postural maintenance activities. In addition, distribution of mechanical work across 14 body joints showed different activation patterns are follwed by lower extremity joints (i.e., ankle, knee, and hip) with changing ship motion. The results of this research provide significant information towards understanding of the impact of ship motion on human performance which will lead to improvements in operational planning and ultimately safety of shipboard personnel. 


\section{Acknowledgments}

I am deeply indebted to my supervisors Dr. Rob Langlois and Dr. Fred Afagh for their continuous support, motivation and guidance throughout my Master's program. I would like to thank them wholeheartedly for providing the fun and stimulating environment which helped me learn and grow.

My thanks go to all the Applied Dynamics Lab members; particularly Nick and Ashley for their cooperation and support throughout this research project. My gratitude goes to former summer student Josh A.Weins for guiding me throughout this project. I would like to thank my best friend Varsha Changai for helping me get through the difficult times, and for all the emotional support and care she provided. Also, I would like to thank my other friends Graham, Simrit, Nihal, Harjeet, Inderjeet, Philip, Angelo, Swati, and other participants for volunteering to be a part of this thesis.

I am grateful to NSERC and the Mechanical and Aerospace Engineering Department for providing the financial support for this research. I would also like to thank the Carleton University Research Ethics Board for their approval on the experimental trials required for the completion of this thesis.

Lastly, and most importantly I wish to thank my parents and family for believing in me and supporting me during my 2 year stay in Canada. To them I dedicate this thesis. 
Dedicated to,

My loving parents, whose support and love made me who I am today. 


\section{Table of Contents}

$\begin{array}{ll}\text { Abstract } & \text { ii }\end{array}$

Acknowledgments iv

Table of Contents vi

List of Acronyms $\quad$ xi

List of Symbols $\quad$ xiv

1 Introduction 1

1.1 Thesis Overview . . . . . . . . . . . . . . . . . . . . . 1

1.2 Motivation ....................... 2

1.3 Problem Statement . . . . . . . . . . . . . . . . . 3

1.4 Research Objectives. . . . . . . . . . . . . . . . 4

1.5 Thesis Organization . . . . . . . . . . . . . . 4

1.6 List of Biological Terms $\ldots \ldots \ldots \ldots$

2 Literature Review 13

2.1 Introduction to Human Balance . . . . . . . . . . . . . 13

2.2 Human Postural Stability _. . . . . . . . . . . . . . . 14

2.2 .1 Physiological Features $\ldots \ldots \ldots \ldots$

2.2 .2 Neurological Features . . . . . . . . . . . . . . 16 
2.3 Muscle Mechanics for Locomotion and Metabolic Energy Expenditure 18 2.3.1 Muscle Classification . . . . . . . . . . . . 18

2.3.2 Skeletal Muscle Physiology . . . . . . . . . . . . . . . . . . 19

2.4 Human Postural Stability Model Development . . . . . . . . . . . 22

2.4.1 Background ........................ 22

2.4.2 Laboratory Experiments and Control Strategies . . . . . . . . 25

2.4.3 Postural Control Strategies Associated with Stepping Response 26

2.4.4 Impact of Motion Waveforms during Dynamic Postural Stability Studies..................... 27

2.4.5 Maintaining Postural Stability in Motion Environments . . . . 29

2.5 Motion Induced Interruption . . . . . . . . . . . . . . . . 29

2.5.1 Development of Different Models for Postural Stability . . . . 30

2.6 Three-dimensional (3-D) Models for Postural Stability Analysis . . . 32

2.7 State of the Art . . . . . . . . . . . . . . . 34

2.7.1 A Six-degree of Freedom (6-DOF) Human Motion Tracking System using Miniaturized Inertial Sensors . . . . . . . . . . . . . 35

2.7.2 Low-cost Motion Capture System for Better Postural Stability Analysis during Onboard Ship Tasks . . . . . . . . . 36

2.7.3 SimMechanics Technology in Simulation of Human Posture (Advancement in Multibody Dynamic Model Development) . . 37

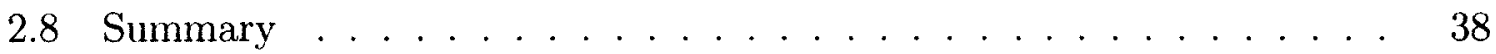

$3 \quad$ Experimental Set-Up $\quad 39$

3.1 Introduction . . . . . . . . . . . . . . . . . . . 39

3.2 Modules for Experimental Set-Up . . . . . . . . . . . . . . 40

3.2.1 MOOG-6DOF Stewart Platform Motion Simulator . . . . . . 40

3.2.2 Opti-track Motion Capture System . . . . . . . . . . . . 46 
3.2.3 Tek Scan Insole Foot Pressure Sensor System $\ldots \ldots \ldots$

3.2 .4 BB1LP Respirometry System . . . . . . . . . . . 51

3.2 .5 Load cell with Force Plate . . . . . . . . . . . . . . . 57

4 Data Collection and Post-Processing $\quad 58$

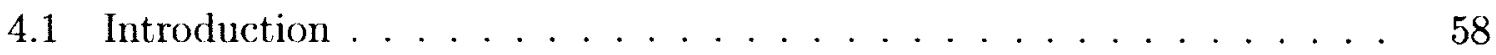

4.2 Test Subject Selection $\ldots \ldots \ldots \ldots \ldots$

4.3 Data Acquisition . . . . . . . . . . . . . . . . . 59

4.4 Data Sampling . . . . . . . . . . . . . . . . . 61

4.5 Data Post-processing . . . . . . . . . . . . . . . 62

4.5.1 Opti-track Data Smoothing . . . . . . . . . . . 62

4.5 .2 Load Cell Data Filtering . . . . . . . . . . . . . 63

5 Multibody Dynamic Model Development 64

5.1 Introduction . . . . . . . . . . . . . . . . . . . 64

5.2 Multibody Dynamic Model . . . . . . . . . . . . . . . . . 64

5.2 .1 Three-dimensional Kinematic Analysis . . . . . . . . 65

5.2 .2 Three-dimensional Kinetic Analysis . . . . . . . . . . 75

5.2.3 Multibody Dynamic Model Comparative Analysis _. . . . 94

5.2.4 Validation of the Multibody Dynamic Model . . . . . . . . 96

5.2 .5 Joint Work Calculations _. . . . . . . . . . . . . . 99

6 Results and Discussion $\quad 100$

6.1 Introduction . . . . . . . . . . . . . . . . . . . 100

6.2 Methods . . . . . . . . . . . . . . . . . . . . . . . . 100

6.3 Variation of Metabolic Energy Levels with Different Ship Motions . . 103

6.3.1 Metabolic Energy Variation with Combined (PRY) Ship Motion 103

6.3.2 Metabolic Energy Variation with Roll Motion . . . . . . . 104 
6.3.3 Metabolic Energy Variation with Pitch Motion . . . . . . . 104

6.3.4 Comparative Analysis for Metabolic Energy Levels . . . . . 106

6.3.5 Male--Female Metabolic Energy Variation with Motion _. 107

6.4 Different Body Joint Contributions to Maintain Postural Stability of the Human Body . . . . . . . . . . . . . . . . . . . . . 109

6.4.1 Mechanical Work Performed by Individual Joints in Combined Ship Motion . . . . . . . . . . . . . . . . . . . 109

6.4.2 Mechanical Work Performed by Individual Joints in Roll Motion 111

6.4.3 Mechanical Work Performed by Individual Joints in Pitch Motion113

6.5 Effect of Ship Motion Severity on Vertical Ground Reaction Force . . 115

6.6 Work-Energy Correlation Results . . . . . . . . . . . . . 116

6.7 Direct Comparison of Mechanical Work with Metabolic Energy . . . 118

6.8 Discussion . . . . . . . . . . . . . . . . . . . . . . . . . . . . 119

6.8.1 Metabolic Energy with Ship Motion . . . . . . . . . . . . 119

6.8 .2 Gender Factor . . . . . . . . . . . . . . . . . 123

6.8.3 Joint Mechanical Work with Ship Motion . . . . . . . . . . 124

6.8.4 Vertical Ground Reaction Force Component Variation with Ship Motion . . . . . . . . . . . . . . 130

6.8.5 Mechanical Work-Metabolic Energy Correlation . . . . . . . 132

6.8.6 Direct Comparison of Mechanical Work with Metabolic Energy 134

6.9 Limitations . . . . . . . . . . . . . . . . . . . . 135

7 Contributions, Conclusion, and Future Work 136

7.1 List of Contributions . . . . . . . . . . . . . . . . 136

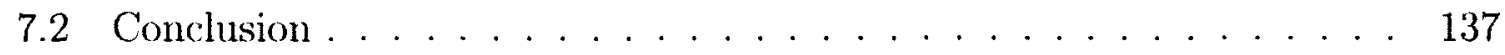

7.3 Future Work . . . . . . . . . . . . . . . . . 138 
A Sample Results for a Single Subject

A.1 Metabolic Energy Variation with Simulated Ship Motion . . . . . 140

A.2 Joint Work Contribution For Different Ship Motions . . . . . . . . 143

List of References 


\section{List of Acronyms}

\section{Acronyms Definition}

\begin{tabular}{ll}
\hline ADP & Adenosine Diphosphate \\
A-P & Anterior-posterior Axis \\
ASCII & American Standard Code for Information and Interchange \\
ATP & Adenosine Triphosphate \\
BMI & Body Mass Index \\
BoS & Base of Support \\
CMOS & Complementary Metal Oxide Semiconductor \\
CNS & Central Nervous System \\
CoG & Centre of Gravity \\
CoM & Centre of Mass \\
& Centre of Pressure \\
CoP &
\end{tabular}




\begin{tabular}{|c|c|}
\hline GRF & Ground Reaction Force \\
\hline GUI & Graphical User Interface \\
\hline HAT & Head, Arms, and Trunk \\
\hline IR & Infra-red \\
\hline ISB & International Society of Biomechanics \\
\hline JCS & Joint Coordinate System \\
\hline LCS & Local Coordinate System \\
\hline LED & Light Emitting Diode \\
\hline L5-S1 Joint & Lumbar-Sacral Joint \\
\hline LSM & Linked Segmented Model \\
\hline MIF & Motion Induced Fatigue \\
\hline MII & Motion Induced Interruption \\
\hline $\mathrm{M}-\mathrm{L}$ & Medio-lateral Axis \\
\hline MS & Motion Sickness \\
\hline NATO & North Atlantic Treaty Organization \\
\hline NMIMC & Navy Medical Information Management Center \\
\hline PRY & Pitch-Roll-Yaw Motion \\
\hline PSD & Power Spectral Density \\
\hline RAOS & Response Amplitude Operators \\
\hline
\end{tabular}


RMR Resting Metabolic Rate

SMS Ship Motion Simulator

STC Standardization and Terminology Committee

xiii 


\section{List of Symbols}

\begin{tabular}{|c|c|}
\hline Symbols & Definition \\
\hline$\alpha$ & Flexion-extension Angle \\
\hline$\alpha_{x_{i}}, \alpha_{y_{i}}, \alpha_{z_{i}}$ & Angular acceleration of $x, y$, and $z$ axes \\
\hline$A_{i}$ & Linear acceleration of $\mathrm{CoM}$ of each segment \\
\hline$A_{j}$ and $\theta_{j}$ & Amplitude and phase response of power spectral density \\
\hline$\beta$ & Abduction-adduction angle \\
\hline$(d L / d t)_{G C S}$ & Moment of inertia along inertial frame of reference \\
\hline$(d L / d t)_{L C S}$ & Moment of inertia along local frame of reference \\
\hline$\ddot{D}_{2}$ & Lateral acceleration \\
\hline$\ddot{D}_{3}$ & Vertical acceleration \\
\hline$\delta_{t}$ & Time rate of change of time \\
\hline$F_{g r f_{x}}, F_{g r f_{y}}, F_{g r f_{z}}$ & Ground reaction forces acting along $x, y$, and $z$-axes \\
\hline$F_{x}, F_{y}, F_{z}$ & Forces acting along $x, y$, and $z$-axes \\
\hline$g$ & Acceleration due to gravity \\
\hline
\end{tabular}




$\begin{array}{ll}\gamma & \text { Axial rotation angle } \\ h & \text { Height of centre of gravity from deck } \\ I_{x}, I_{y}, I_{z} & \text { Principle mass moments of inertia about } \mathrm{x}, \mathrm{y}, \text { and z-axes } \\ l & \text { Half the base of support } \\ l / h & \text { Tipping coefficient } \\ M & \text { Number of components used for approximation of contin- } \\ \vec{M}_{j o i n t} & \text { uous spectrum } \\ M_{g r f_{x}}, M_{g r f_{y}}, M_{g r f_{z}} & \text { Ground reaction moments acting along } x, y, \text { and } z \text {-axes } \\ M_{x}, M_{y}, M_{z} & \text { Moments acting along } x, y, \text { and } z \text {-axes } \\ \eta_{4} & \text { Instantaneous roll angle } \\ \ddot{\eta}_{4} & \text { Instantaneous roll acceleration } \\ \vec{O}_{C o M} & \text { Cosition vector which defines relative position between lo- } \\ q_{t} & \text { Time of mass location } \\ \phi, \theta, \psi & \text { Specific ship motion } \\ R_{G} & \text { Euler angle sequence } \\ & \end{array}$




\begin{tabular}{ll}
{$\left[T_{A T 2 G}\right]$} & Transformation matrix from anatomical to global axes \\
$V_{\mathrm{O}_{2}}$ & Volume of $\mathrm{O}_{2}$ \\
$V_{C O_{2}}$ & Volume of $C \mathrm{O}_{2}$ \\
$W_{B o d y}$ & Mechanical work done by human body \\
$W_{\Delta \theta}$ & Amount of work done by joints in changing orientation \\
& from $\theta_{i}$ to $\theta_{i+1}$ \\
$\left(x_{l}, y_{l}, z_{l}\right)$ & Local coordinates \\
$\left(X_{G}, Y_{G}, Z_{G}\right)$ & Global coordinates \\
$\left(\omega_{x}, \omega_{y}, \omega_{z}\right)$ & Angular velocities about different axes \\
\hline
\end{tabular}




\section{Chapter 1}

\section{Introduction}

\subsection{Thesis Overview}

Postural stability is considered to be a multidisciplinary field which incorporates science, environmental factors, and various other disciplines in order to reduce falling risks to the extent possible. Human postural stability remains a topic of concern for naval as well as commercial fleets where shipboard personnel are constantly exposed to the postural hazards associated with constant deck motion.

The long-time operational activities in maritime environments can increase the chances for motion induced fatigue (MIF) and motion sickness (MS) to a great extent. Motion induced fatigue is considered as a major biodynamic obstacle, which is responsible for injury/accident occurrences by degrading human performance during maritime operations. Wertheim (1998) described that ship motions contribute to lack of motivation of crewmembers by increasing their metabolic energy requirements which can result in motion sickness and motion fatigue, thus being responsible for generating various balance related problems $[1,79]$.

This research was focussed on determining a better correlation between mechanical work done by humans with the amount of metabolic energy expended by them, in order to predict the occurrence of motion induced fatigue (MIF) and motion disorders. 
The detection and possible minimization of motion induced fatigue (MIF) can provide valuable safety guidelines for planning of successful deck operations as well as insights for more suitable ship design.

In this investigation, basically three types of data were obtained: foot pressure data, body kinematics, and metabolic energy data. These data were obtained for various levels of simulated deck motion that is representative of typical ship motion environments. These data were further analyzed to find a correlation between mechanical work performed by humans and the metabolic energy expended by them while maintaining balance to avoid falling. By measuring joint motion and determining the associated muscle forces, the mechanical work involved in postural control can be determined. This in turn can be compared to the measured total metabolic energy to determine the extent to which postural control affects energy consumption. Correlating mechanical work to deck motion allows development of a simple model that is indicative of expected level of fatigue for prevailing ship operating conditions.

\subsection{Motivation}

This research is expected to make an important contribution to understanding of postural stability problems experienced by the commercial as well as military maritime communities, resulting in overall improvement of crew performance by better operational planning. This is important because it allows warships to perform with minimum degradation of mission effectiveness even under adverse circumstances [4]. Moreover, recent literature (Riola J.M. et al) indicates that approximately 10 million people travel on more than 230 cruise lines each year throughout the world [2]. So, establishment of a better comfort level for passengers is considered as a prominent task of naval architecture for efficient ship design. Better ship design is expected to put more emphasis on ensuring safety of human participants during routine as 
well as emergency operations. Since crewmembers deal with numerous tasks for various operations during routine missions, ship motions are considered to be primarily responsible for limiting performance abilities of crewmembers by increasing their discomfort level. As evidence, data from Navy Medical Information Management Center (NMIMC) indicated that 4,89,266 navy recruits were diagnosed with motion sickness occurrences between 1982 to 1992 [3].

In the naval community, longer maritime operations decrease performance of crewmembers and ship operators, which ultimately lead to impairment of their task ability in motion environments. The main reason behind this is increased occurrence of motion induced fatigue (MIF) and motion sickness (MS) which are associated with prolonged exposure to motion rich environments. The situation becomes even more critical in emergency circumstances when a percentage of the crewmembers, who are normally ready to respond, effectively reduces. These effects collaboratively increase the vessel costs and reduce fighting ability during naval operations by decreasing overall ship efficiency $[6,7]$. Hence, there is a requirement for effective human factors design for enhanced efficiency and safety of personnel as well as the vessel as whole [7].

The results of this study can be used for providing valuable information regarding proper shift work scheduling, maintaining adequate balance for work-rest ratio, health, and nutrition demands and enhanced vessel design for better efficiency during various ship tasks.

\subsection{Problem Statement}

The research is based on the hypothesis that metabolic energy expenditure increases with ship motion severity. Elevated metabolic levels result in motion induced fatigue (MIF) and similar motion disorder occurrences. This research provides better understanding of fatigue and other disorders in order to increase the safety and efficiency 
of vessel's crew.

\subsection{Research Objectives}

The broad objective of this research is to gain a thorough understanding of maintenance of postural stability of crewmembers and ship operators during elevated sea conditions.

Maintenance of postural stability becomes difficult when the human body is continuously exposed to ship motion. This is because it takes time to adjust the body with continuous and prolonged motion variation. Increased motion variations are expected to increase levels of metabolic energy expenditure as compared to energy requirements of the human body while working in stable environments. Performing tasks within motion rich environments becomes more challenging as the body experiences increased musculoskeletal loads due to repetitive postural adjustments in order to retain its balance during ship deck motion. Every time there is a postural adjustment to overcome ship motion, there is increased muscle tension/compression due to muscle fibre activation, resulting in increased metabolic energy expenditure. This energy expenditure goes on increasing with long time ship operations, which is cumulative with continuing exposure $[1,5]$. Therefore, there is need to investigate and understand the major factors contributing to motion disorder occurences during rough sea states.

\subsection{Thesis Organization}

The thesis is organized in the following sequence.

In Chapter 1, we shed light on research objectives, the problem statement, and 
the project motivation. This is preceded by a brief introduction to human postural stability and its relationship with ship operability in the context of quantitative analysis of human performance in motion environments.

Chapter 2 presents neurological and physiological aspects of postural stability to correlate human factors with stability phenomena in maritime environments. A review of existing postural stability models and current approaches used for investigating shipboard postural stability criteria is presented. This is followed by a brief discussion of ongoing developments in the postural stability field aimed at accurate humanoid balance understanding.

Chapter 3 covers key elements about the experimental set-up used in this investigation. Each of the five modules associated with the experiment, their calibration, and other associated aspects are discussed. This chapter includes detailed description of the experimental set-up for skeletal motion data, deck interface forces, and respirometry data collection with a brief discussion of motion profile generation for the ship motion simulator (SMS).

Chapter 4 provides information about data collection and post processing of the recorded data. This chapter explains participant selection criteria, the data acquisition procedure, data sampling, and data smoothing prior to actual data reduction and analysis. In other words, this chapter covers preliminary steps required prior to actual data analysis; including data gap filling, data sampling, and data synchronization to ensure the highest data quality prior to proceding with data analysis.

Chapter 5 represents a three-dimensional dynamic model of the human body developed using rigid multibody dynamics. Using inverse dynamics, this model is applied to evaluate the mechanical work performed by the human body at different joints while maintaining balance during severe ship motion conditions. This chapter basically outlines 3 topics: three-dimensional kinematics of the human body, three-dimensional kinetics, and resultant joint forces and moments. This is followed by estimating the 
total mechanical work performed by the body. Moreover, by considering different solution approaches, the most comprehensive approach for joint work calculation is presented. Finally, validation of the three-dimensional multibody dynamic model is presented.

Chapter 6 presents computational methodologies applied for different sets of collected data. Various data analysis techniques are applied and discussed for these different data sets. Further, results of different data sets are presented together for detection of mechanical work done and metabolic energy expended. The results of mechanical work from the proposed multibody dynamic model are correlated with measured bio-mechanical energy expended by humans while performing various activities in motion-rich environments. Detailed analysis of outcomes is presented for detection of motion induced fatigue effects on humans as they are affected by ship motion severity.

Chapter 7 concludes with a brief summary of the research conducted. Major contributions of the research are highlighted and discussed. Further, future research possibilities, prospects, and the potential developments are outlined and suggested.

\subsection{List of Biological Terms}

- Agonistic Muscles

Muscle groups that allow the body segments to undergo some movement through the contraction process (due to a sliding pattern generated by actinmyosin fibres). These muscles are regarded as 'prime movers'.

- Antagonistic Muscles

Muscles that work in the opposite direction to that of the corresponding agonistic muscles. 
- Coronal Plane

The plane which divides the body into ventral and dorsal (front and back) sections. It is also sometimes called the frontal plane.

- Sagittal Plane

A vertical plane that divides the body into right and left halves by passing from the front to the back of the body.

- Transverse Plane

A horizontal plane that divides the body into superior and inferior sections and is perpendicular to both coronal and sagittal planes.

- Adenosine Triphosphate (ATP)

A high-energy molecule, which is regarded as energy currency of the cell, and is responsible for transporting chemical energy within cells for metabolism.

- Adenosine Diphosphate (ADP)

A nucleotide, composed of adenosine and two linked phosphate groups converted to ATP for energy storage.

- Central Nervous System

Comprises both brain and spinal cord, responsible for sending, receiving, and interpreting information from all body parts. It coordinates body activities by responding to external stimuli.

- Biosensor

A device that detects, records, and transmits information regarding physiological or biological activity or process. An example is the blood glucose sensor. 
- Motor Neuron

An efferent neuron in the nervous system responsible for controlling various muscle activities by facilitating muscle contraction and relaxation.

- Tendon

Fibrous connective tissue that connects muscles to bone. These are made up of collagen and work together with muscles to allows contraction and relaxation processes to occur.

\section{- Mitochondria}

Mitochondria are membrane bound organelles which are regarded as power centres of cells and are responsible for providing energy to cells required for division, movement, and production of secretary products.

\section{- Sarcomere}

The repeating subunits of myofibril which are delimited by the Z-bands along the myofibril length. The muscle fibrils within Z-bands consist of contractile properties.

- Neuron

A specialized impulse-conducting cell of the nervous system which consists of the cell body and its processes (axon and dendrite) for sending and receiving impulses throughout the body.

- Muscle Spindle

Muscle spindles are sensory receptors within the belly of the muscle, which detect change in the muscle length. This change in muscle length information is conveyed to the central nervous system (CNS) through neurons, which is required for the reflex mechanism that maintains body posture. 
- Muscle Fibres

Muscle fibres are defined as many long multi-nucleated muscle cells or myofibrils that are arranged in bundles called fascicles. Fascicles are further grouped together to make a muscle belly.

- Lumbosacral (L5-S1) Joint

The human body joint between the terminating lumbar vertebrae (L5) and the first sacral (S1) segment of the spinal cord.

\section{- Pneumotachometer}

A device that converts the flow of gases through it into proportional pressure difference signal between inhaled gas and the outside environment.

- Centre of Gravity (CoG)

The point through which the resultant of gravitational forces on a body acts.

- Centre of Mass (CoM)

The weighted average of the location of all masses in the body or group of bodies.

- Centre of Pressure (CoP)

Point on a body where the total sum of a pressure field acts, which consists of forces and no moments about that point.

- Anterior-posterior (A-P) Direction

The direction of the anatomical axes system, which is directed from front (anterior) to back (posterior) of the body segment. 
- Medio-lateral (M-L) Direction

The direction of anatomical axes, directed sideways through the body segment from the medial to lateral side of it.

- Axial Direction

Direction of anatomical axes along the length of the segment, representing the vertical direction of the body segment.

- Proximal

The proximal location is situated nearest the origin or point of attachment of the body segment or bone.

\section{- Distal}

The distal location is situated farthest from the origin or point of attachment of the body segment or bone.

- Floating Axis

A rotating axis in the joint coordinate system (JCS) defined by the cross product of the distal segment longitudinal axis with the proximal segment medio-lateral axis. It is oriented in the direction perpendicular to the plane formed by these directed axes.

\section{- Semicircular Canal}

Any of three tubular and looped fluid-filled structures of the inner ear, which are functioning together in order to maintain a sense of balance in the human body. 


\section{- Otoliths}

Any of the three small oval calcareous bodies which are located within the inner ear and concerned with sensing gravity and movement of the human body.

- Flexion-Extension

Act of bending a joint (flexed position) and extending it.

- Adduction-Abduction

Movement of body segments towards and away from the central axis of the human body.

- Axial Rotation

Rotation about the length of the axis.

- Muscular Fatigue

Temporary reduction in the muscle strength (either power or endurance) is called muscle fatigue. In this condition, muscle loses its capacity to undergo maximum contraction for force generation.

- Motion Induced Fatigue

A motion disorder which is caused by increased muscular effort to maintain a balanced state within a motion environment.

- Motion Sickness

State of uncomfortable dizziness, nausea, and vomiting which is experienced by people when their sense of balance and equilibrium is disturbed by being in constant motion for prolonged periods of time. 
- Sopite Syndrome

A motion induced neurological disorder, which can be related to fatigue, drowsiness, and mood changes caused by prolonged exposure to a motion environment.

- Response Amplitude Operators (RAOs)

RAOs are effectively the transfer functions used for determining the effects of sea state on ship motion.

- Sensels

Sensing elements present in the foot insoles used to measure the foot pressure are called sensels. The resistance of sensels is inversely proportional to the load applied to it. 


\section{Chapter 2}

\section{Literature Review}

\subsection{Introduction to Human Balance}

The problem of human posture recognition concerns different research fields and has become of special interest in the last two decades. Human posture is defined as delicate interaction between multiple forces as well as torques, which vary according to body orientation, motion of different body segments, and environmental conditions [8]. When the human body undergoes perturbations, various forces including gravity,

muscle, and inertia forces; which appear due to angular velocity and acceleration of various body segments are developed. When non-muscular forces, which are generated due to disturbances to human posture, become compensated with muscular forces, there is restoration of human balance. Therefore, evaluation of the contribution of both muscular as well as non-muscular forces, which control the posture, is necessary in order to understand the posture recovery mechanism [8]. Maintenance of human postural stability is accomplished by integrated control structures in the complex musculoskeletal system. The human body is comprised of approximately 750 muscles, which activate a control system with 200 degrees of freedom, to maintain human balance $[9,11]$. Different body joints undergo different degrees of freedom in order to orient the body in such a way that balance can be maintained. 


\subsection{Human Postural Stability}

\subsubsection{Physiological Features}

Postural balance is defined as the major integral component for all tasks that require the body to undergo a change in stability [41]. According to Shumway-Cook and Woollacott [80], balance control is defined as interaction between individual characteristics, task factors, and environmental conditions (Fig. 2.1).

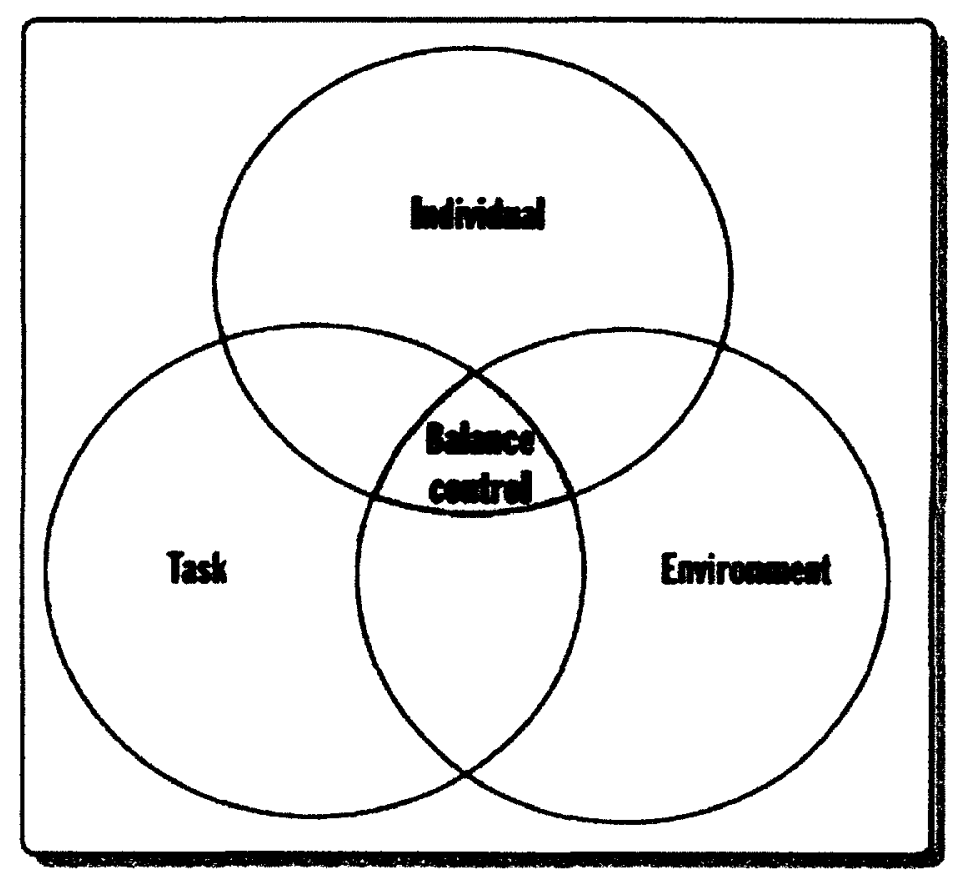

Figure 2.1: Conceptual model of the balance control strategy [80].

Spatial orientation of the human body can be represented on the basis of the following three anatomical planes as shown in Fig. 2.2:

- Transverse Plane: The plane which is parallel to the ground and bisects the human body into superior (head) and inferior (lower extremities) segments [45].

- Coronal Plane: The plane which is perpendicular to the ground and divides the body into front (ventral) and back (dorsal) segments [45]. 
- Sagittal Plane: The plane which is also perpendicular to the ground and bisects the human body into left and right halves and also defines a plane of symmetry for a person [45].

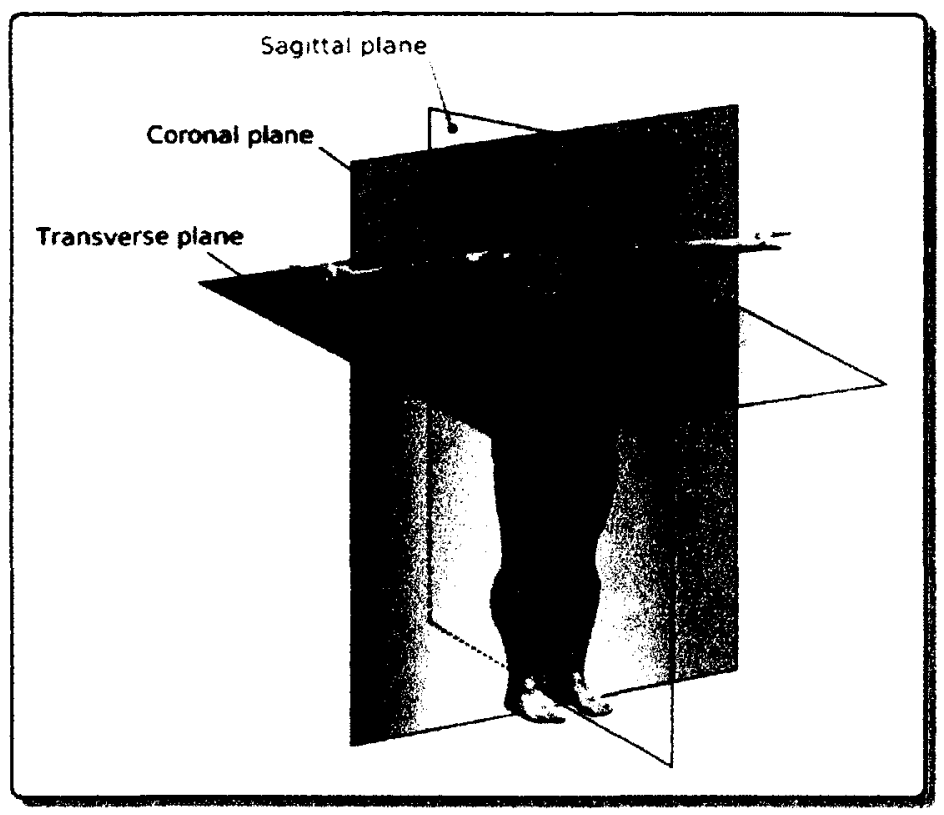

Figure 2.2: Anatomical planes for the human body [45].

As discussed under Section 2.2.2, there are three primary sensory mechanisms, which work together to maintain postural stability: vestibular, proprioception, and vision. Each system contributes to provide unique information regarding the human motion as well as the surrounding environment. The information from all three systems is integrated together, which is further concatenated by the central nervous system (CNS) to evaluate overall postural state. This is required for determination of the mechanical response of the humans. Each system provides information regarding a different type of postural disturbance, to which it is most sensitive. This information from each system plays a very important role in maintaining overall postural stability, which vary in relation to various motion characteristics, i.e., amplitude, frequency, and envirommental factors.

There are many factors that can affect the postural sway strategy: 
- Type of motion as well as supporting surface.

- Age (as elderly people are more prone to falling than younger people).

- Type of task to be executed.

- Obesity.

- Deficits in the postural system (neuromuscular system disorders).

- Stance width (wider stance facilitates postural stability maintenance).

- Level of past experience with motion enviromments.

\subsubsection{Neurological Features}

\section{Sensory Input Systems}

The complex process of postural stability can be defined on the basis of interaction between three different sensory input systems of the CNS as outlined below:

\section{Vestibular System:}

The vestibular system is one set of sensory mechanisms that contributes to maintenance of postural balance and movement in most mammals. The vestibular system possesses two basic components: semicircular canals and otoliths, which sense the rotational and translational movements, respectively. The vestibular system transmits signals to neural structures (which control eye movement) and to muscles (which keep the upright posture maintenance). In this way, the vestibular system behaves as a "gyro" to sense linear as well as angular accelerations $[41,46]$. 


\section{Proprioception System:}

The proprioception system is another set of sensory mechanisms, which deals with muscle contraction variation in response to external forces by utilization of stretch receptors in muscles in order to keep all the joint positions as required. The proprioception system works along with the vestibular system to transmit the information regarding change in muscle length, tension, pressure and noxious stimuli via proprioceptors [41]. The muscle spindles which act as complex proprioceptors inform other neurons of muscle length and tension, and this input from spindles can be provided to the brain in order to update changes in joint angle and position. In this way, the proprioception system contributes to maintenance of postural stability via muscle spindles. In all, the proprioception system behaves as a "hub" of biosensors which are involved in sensing position and velocity of all body segments and their contact with external objects [41].

\section{Visual System:}

Another very important set of sensory mechanisms, which helps in maintaining balance, is the visual system. The visual system automatically enables humans to process visual information. With this mechanism, representation of the surrounding world takes place by interpreting the visual light information from the eyes as a sensory signal which can be further processed by the central nervous

system and contributes to the humanoid stability maintenance process. Therefore, the visual system is involved with better planning of locomotion for bipeds stability [41].

\section{Motor Reactions}

Primary motor reactions for balance maintenance are activated through the muscles of the lower extremities: feet, legs, and trunk. Motor neurons are present in muscles 
of the feet, legs, and trunk which carry impulses from brain to the body and control movement of muscles as well as secretion of glands which are further required for maintenance of postural stability [46].

\section{Central Nervous System}

The central nervous system (CNS) is required for integration of both sensory and motor process inputs for controlling human movements. Physiologically, the CNS system is the major processor unit which includes the brain and spinal cord. It integrates the information received from motor reactions and the three sensory input systems and maintains stability by coordinating activity of all body parts. Specifically, the CNS processes information from three sensory inputs and selects the most accurate information related to specific tasks as well as surrounding environments [46]. On the basis of input received, it generates a response and sends it back in order to maintain balance in motion enviromments by avoiding falls. In this way, the CNS is assigned to organise sensory input information from the three systems, select the motor responses in order to stabilize posture, and prevent falls depending upon motion severity and environmental factors.

\subsection{Muscle Mechanics for Locomotion and Metabolic Energy Expenditure}

\subsubsection{Muscle Classification}

Muscles are regarded as the body tissues consisting of cells responsible for force as well as moment generation. Muscle cells are generally cylindrical with a diameter between 10-100 $\mu \mathrm{m}$ and are a few centimeters long. Characteristics of muscles are dependent upon the organization of muscle fibres. These fibres run throughout the whole length 
of the muscle, tending to become oblique at the line of action of the muscle. Together muscles and bones maintain structure and movement of the body [39]. Muscles can be categorized into three categories depending upon their particular characteristics:

- Skeletal Muscles:

Skeletal muscles are voluntary muscles, which comprise approximately $40 \%-$ $45 \%$ of human body weight. These muscles are attached to bones via tendons and are major source of locomotion of human body [39].

- Smooth Muscles:

Smooth muscles are involuntary in nature and are found in the lumen of blood vessels, urinary tract, as well as the gastrointestinal tract. These muscles perform the function of controlling lumen of these tubes and generation of peristaltic waves required for digestion of food. Unlike skeletal muscles, smooth muscle cells are less common [39].

- Cardiac Muscles:

As the name suggests, cardiac muscles make up the bulk of the heart mass and are involuntary in nature. These muscles are responsible for heart beat generation by causing the heart mass to undergo contraction [39].

\subsubsection{Skeletal Muscle Physiology}

Skeletal muscles are long, thin, spindle-shaped adaptable tissue that are responsible for locomotion in the human body (Fig. 2.3). Skeletal muscles also possess blood vessels and muscle cells called muscle fibres, which are $30 \mathrm{~cm}$ long with a diameter between 10-100 $\mu \mathrm{m}$. Muscle cells are multinucleated in nature with many mitochondria at each cell. The most prominent building block of skeletal muscle is myofibril which is a long rod shaped element responsible for the muscle contraction phenomenon. 


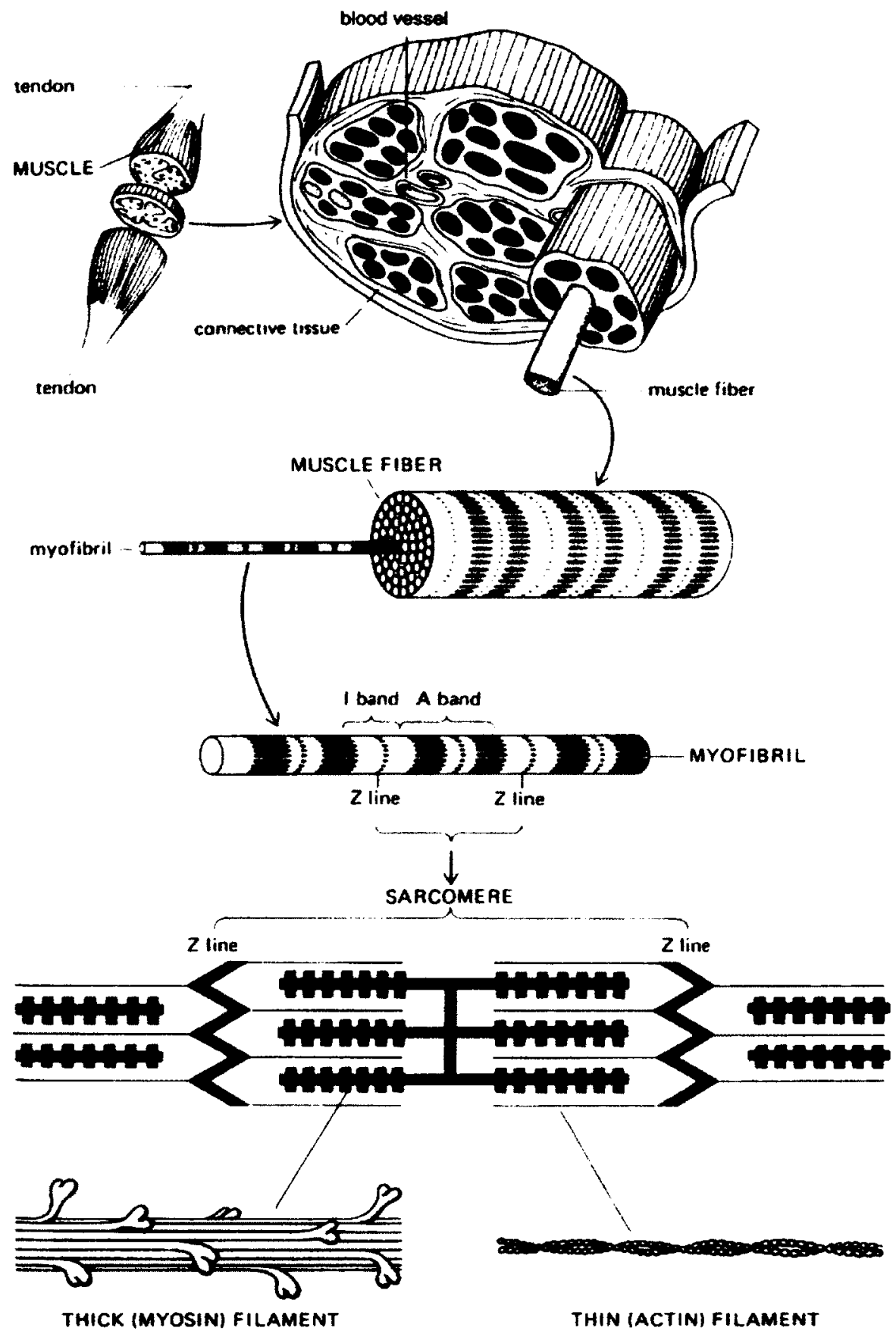

Figure 2.3: Skeletal muscle structure overview [39]. 
Myofibrils possess a regular banded structure with a repeated pattern which is called the sarcomere. The sarcomere is composed of a definite arrangement of even smaller subunits:

i. Thick filaments known as myosin myofilaments.

ii. Thin filaments called actin myofilaments.

As shown in Fig. 2.3, both actin and myosin filaments are arranged in a crossbridge layout due to interpenetration of the actin-myosin complex within the sarcomere. Formation of the actin-myosin complex is an energy utilizing process, which can be provided by the breakdown of the adenosine triphosphate (ATP) molecule. Breakdown of ATP into adenosine diphosphate (ADP) and phosphate ions releases very high energy which causes the myosin head to attach to actin, maintaining a sliding motion pattern, in which both myofilaments (actin and myosin) slide over each other and allow muscles to undergo contraction. This muscle contraction phenomenon is due to direct conversion of chemical energy (ATP molecule) into motion [39]. In other words, this phenomenon is the basic physiological process through which metabolic energy is expended by different muscles within the human body while performing any mechanical task. Thus, while maintaining a balanced state during different tasks, the greater the generated muscle tension, the greater will be activation of muscle fibres. This will lead to higher consumption of ATP in order to produce sliding movement between actin and myosin myofilaments, resulting in greater metabolic energy expenditure levels. 


\subsection{Human Postural Stability Model Development}

\subsubsection{Background}

A number of researchers began working on modelling the movement of human posture during standing conditions as early as 1959. The result was to develop different techniques to measure these movements in the following years [16]. Many models have been developed so far to describe human postural stability, which vary from a single segmented inverted pendulum to full-articulated multi-link models [17]. The initial objective of development of these human postural stability models was to understand loss of balance. More recent developments have extended this research to quantify the effect of ship motion environments on human postural stability in order to understand human performance onboard ships. For example; during navigational tasks, crew members are supposed to perform various physical as well as mental tasks. If due to external perturbations crew members are not able to perform their respective tasks properly, it will affect the overall efficiency of the ship both economically as well as performance wise. Crew postural instability affects both types of vessels: commercial and military. This results in increased cost of operation for commercial vessels while it decreases the fighting ability of naval vessels [18]. All of this has motivated the development of a number of postural stability models in recent years. Development of a simple inverted pendulum was a result of these efforts. In 1970 , McGhee and Kuhner [16] developed one of the earliest single link inverted biomechanical models. The objective of this work was the development of legged vehicles as well as controlled prosthetics for the medical field. Development of several other inverted pendulums came into existence during that period of time. For example, a model was developed by Nashmer which considered feedback control strategies in order to model human postural stability [17]. In 1970, Hemami introduced his own inverted pendulum approach, which was based on development of a theoretical control scheme for 
maintaining postural stability in biped gait [18]. The complexity of human postural stability models increased with the progress of time, technology, and knowledge as well as exposure in the area. In 1976, there was one major development in this field, when Geurssen represented a two-link inverted pendulum as a model to study human postural stability [19]. This model presented the human body as two links. The lower link modelled the legs while the upper segment represented the head, arms, and trunk (HAT). Each segment was characterised by its centre of mass (CoM) as well as its length [19]. This model was able to distinguish the difference between the centre of mass (CoM) of the body and the centre of pressure (CoP) in postural stability analysis. Later on, Hemami extended his work by developing a three-link humanoid, which comprised three segments: shanks, thighs, and upper body (HAT) with control actuators to control position and velocity at each joint [19]. Since this model was comprised of three linked segments it was suitable for investigating various postural stability tasks at different body postures; particularly sitting, standing, bending, and squatting [20]. In 1980, Hemami's approach was extended by Koozekanani by adding a triangular foot as a support base for better stability analysis. Free body analysis techniques coupled with kinematic constraints were used by Koozekanani to estimate the CoM and CoP variation associated with a kinematic data set. The model was validated successfully by using human subjects with a televised body tracking system as well as force plates for determining pressure distribution. It was suggested that postural stability of the human body can be better represented by modelling it with a three-dimensional approach. In 1981, Koozekanani and Stockwell further extended their model to a five-link model [14,21]. Later on, in 1982, Kodde developed such a model based on a three-dimensional approach, which comprised two separate planar models for both the frontal and sagittal planes [23]. This model was used in successful quantification of CoM based on collected CoP data from force plates. During the $1980 \mathrm{~s}$ and $1990 \mathrm{~s}$, models became more and more complex with advancement of 
computational research techniques. In 1991, Riley developed a 11-link segmented

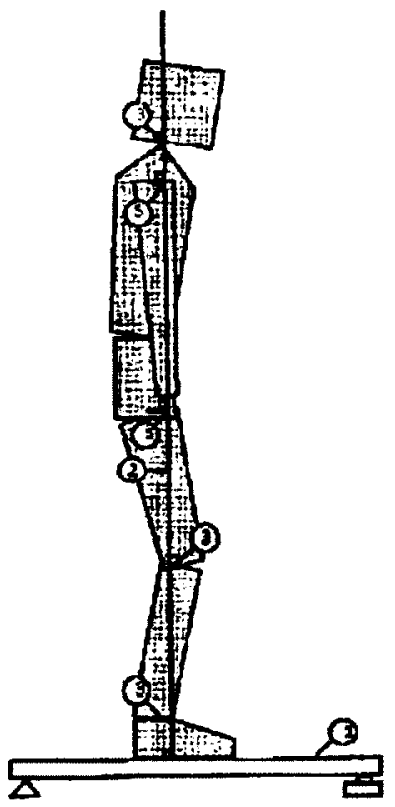

Figure 2.4: Riley's 11 segment postural model [24].

model with six degrees of freedom at each joint (Fig. 2.4). Experimental studies were equipped with a visual tracking system as well as a force plate for calculation of centre of gravity displacements using the proposed model [24]. In 1998, Winter developed one of the most complete models to date, which comprised 14 segments. Unlike Riley's human body representation as a single link, it represented trunk as four segments. This model was helpful in obtaining better correlation of muscle stiffness with postural stability control of humanoids during standing posture. In modern postural stability models complexity has been increased further by incorporating control systems, which are implemented by the central nervous system during different tasks such as standing, walking, etc. In this regard, Patton, Humphrey, and Tian have used two, three, and four-link models respectively to implement different control strategies which increase complexity [25-27]. 


\subsubsection{Laboratory Experiments and Control Strategies}

Experimental investigation is a major requirement in validating any postural stability model that is developed. Intricacies of human postural stability control can be better understood by studying it for a wide range of frequencies, amplitudes, and waveforms. Motion platforms are used in experimental work to simulate various motions under which postural stability is investigated. Human responses to perturbing accelerations are measured as ground reaction force (GRF) as well as kinematic data. Since there is variation in motion characteristics, selection of ideal motion waveforms for better postural control was a bit debatable. For this, in 1980 Ishida represented the concept of using pseudo-random acceleration disturbances for postural studies [30]. A television measurement system and force platform were incorporated in this experiment for recording ankle and hip angles as well as centre of pressure, respectively, when subjects were subjected to a series of random motions (above $0.5 \mathrm{~Hz}$ ). The objective was to develop frequency response functions for torque as well as segment angles generated due to perturbing accelerations. In this way, a transfer function for ankle angle to ankle torque was fitted into collected data and postural controller for a single link inverted pendulum was proposed [30].

During recent years, the field of postural stability modelling has advanced considerably due to implementation of new techniques involving both internal and external effects. For example, Humphrey and Hemami [26] represented a 3-link model with attachment of massless feet that incorporated a large muscle actuator system having nine different muscle groups. Moreover, in this study a vestibular system model was also incorporated in order to relate postural response to actual sensed accelerations rather than externally applied motion inputs [26]. 


\subsubsection{Postural Control Strategies Associated with Stepping Response}

In 1985, the single link inverted pendulum model proposed by Ishida was revised by Peeters, who revised it using a double input controller for a double inverted pendulum with links representing trunk and legs individually. Although the model was not successful in accurate reproduction of experimental results when used for high frequency motion, it clearly demonstrated non-linearities for high frequency motion preturbations. Peeters developed an improved postural stability quantification method based on these nonlinear postural control effects. Basically, evaluation of postural stability from mean postural sway during quiet standing position was investigated. Peeters further proposed that falls in the elderly can occur due to unexpected postural disturbances and therefore more accurate postural stability quantification methods can be provided by measurement of postural sway during support surface disturbances [8]. His model possesed a linear parametric control system coupled with a saturation function. Every time the perturbation magnitude causes a subject to take a further step in order to maintain balance, there is coincidence between the saturation point and an abrupt change in balancing strategy. Kinetically, this saturation point can be comparable to $\mathrm{CoP}$, which further exceeds the limits of the anterior-posterior support base (which span between the front of the toes and the back of the heels). In the late 1990s, Maki, Patton, and Pai $[25,31,32]$ conducted studies for development of a better characterization of step response threshold. Their modelling approach was more oriented towards development of a dynamic model of CoM displacements for evaluation of step inducing conditions in motion environments compared to a static modelling approach. This approach was proposed by taking into consideration the velocity of the CoM along with its position. The basis of this approach was its hypothesis that the body will act to maintain balance when its CoM reaches the dynamic 
stage where the ankle joint torque will be unable to prevent it from exceeding the base of support limits. This approach was verified by experimental investigations, which demonstrated that a dynamic threshold is a better predictor of stepping response compared to a static threshold. In 2003, experiments were also performed by Millie in order to reinforce the concepts of dynamic threshold put forth by Maki, Patton, and Pai $[25,31,32]$. According to them, most experimental studies were limited to only the sagittal plane; however some work was done toward understanding the difference between the coronal plane and the sagittal plane. In this regard, Maki and Mcllroy $[32,34]$ found that the coronal plane segment configuration geometry results in more complex stepping responses compared to the sagittal plane configuration [32]. This is because the coronal (or frontal) plane possesses a unique set of dynamic constraints, which need to be quantified in order to develop an accurate three-dimensional postural stability description.

\subsubsection{Impact of Motion Waveforms during Dynamic Postural Stability Studies}

In 1994, Maki and Mcllroy [32,34] proposed that postural stability response is not easy to understand even by fully defining perturbation motion waveforms. $\mathrm{U}_{\mathrm{p}}$ to that point, only ramp type motions were used to provide perturbing accelerations for platform disturbance studies. Maki and Mcllroy [32,34] further proposed that destabilizing forces that can be developed due to perturbing accelerations and ramp type motions (which provide perturbing accelerations) basically are comprised of two separate destabilizing incidents. The first incident corresponds to acceleration of the experimetal motion platform while the other can be provided by the deceleration stage. Experimental studies have further verified that the deceleration stage has great impact on postural response. In case of a predictable motion, the postural stability 
strategy can be altered by considering the fact that inertial effects during decelerations counteract the destabilizing forces, which can be imposed by onset accelerations. In 2001, Maki and Mcllroy's approach $[32,34]$ was further extended by Brown in order to evaluate the impacts of wave form shape [35]. Although Brown also proposed that ramp type displacements were comprised of two separate destabilizing incidents, he also suggested that it is very important to fully define characteristics of accelerations occurring during these phases. Thus, for his experiments, he used three types of waveforms: ramp, ramp-parabolic, and sinusoidal (Fig. 2.5). Each type of waveform reproduced a unique neuro-muscular response which reinforced the concepts of Maki and Mcllroy that it is necessary to fully define the characteristics of the perturbing accelerations in order to understand the consequent postural stability reactions [35].

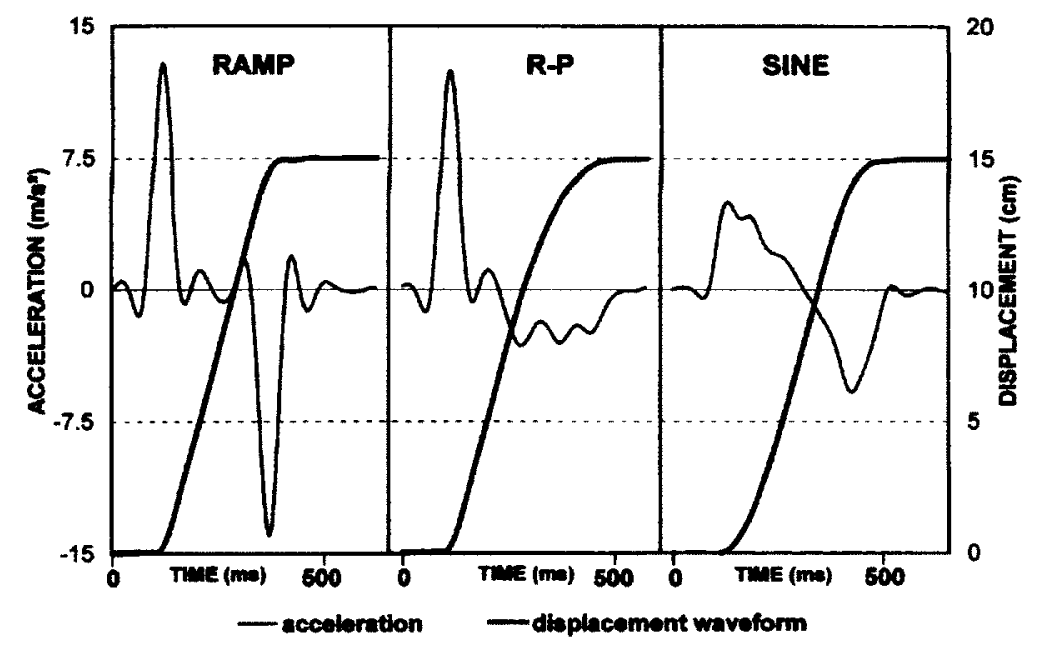

Figure 2.5: Three types of motion waveform proposed by Brown [35]. 


\subsubsection{Maintaining Postural Stability in Motion Environ- ments}

An average person is subjected to a great variety of postural stability challenges throughout his/her life during daily activities. Walking, standing on buses, and standing on ships are common examples that can be studied in detail in order to understand postural stability phenomena. Considering such perturbations in postural stability experimental studies can provide greater opportunity to correlate experimental results with theoretical knowledge that is developed through postural stability model development. However, to the best knowledge of this investigation, so far no postural stability study has been conducted related to trains and buses, whereas postural stability during various shipboard tasks is a main concern of the research community. So far, investigators have considered applications of postural stability models in ship tasks as well as in naval operational industries in order to better understand postural stability and its impact on human performance at sea. Besides this, there are also numerous studies of postural stability in non-moving enviromments. Chiou presented a detailed study of postural stability risks during various working environments in $1998[1,79]$. In this study, tasks such as walking, standing, and bending were investigated with respect to various environmental factors such as surface contamination, firmness, and workload.

\subsection{Motion Induced Interruption}

Motion induced interruptions (MIIs) are defined as instances when personnel temporarily abandon their allotted tasks to try to maintain their upright posture as the result of losing their footing due to severe ship motion [11]. MII rate is an important measure of performance degradation quantification during various ship as well 
as other operational tasks. This is because severe ship motions force a crew member to initiate a variety of actions such as grabbing a support, taking a step, or stopping their task in order to maintain balance.

This concept was first introduced in 1984 by Baitis and Applebee which referred to this phenomenon as Motion Induced Interruptions [36]. Today, MII rate is considered as an important measuring scale of ship motion severity. North Atlantic Treaty Organization (NATO) defines one MII per minute as a reasonable risk level for most of shipboard tasks.

A variety of postural stability models have been used to investigate MII. These models are very important for both naval as well as commercial ship operations, as they basically predict the incidence of loss of stable posture by humans when they experience an acceleration exceeding a threshold.

\subsubsection{Development of Different Models for Postural Stability}

This section presents a review of postural stability models available in the literature:

\section{i. Graham Model}

A very simple mathematical model for postural stability was developed by Graham [12]. It estimates the number of MIIs per minute in a motion environment during standing state [13]. In addition, Baitis and Applebee [36] extended MII prediction as a function of lateral accelerations in the plane of the deck, which seems to be a suitable measure for quantification of ship motion effects on individuals [13]. Graham et al [15] provide equations to predict the occurrence of tipping for human standing posture such that tipping is expected, when:

$$
\left(\frac{1}{3} h \ddot{\eta}_{4}-\ddot{D}_{2}-g \eta_{4}\right)-\frac{l}{h} \ddot{D}_{3}>\frac{l}{h} g
$$


or

$$
\left(-\frac{1}{3} h \eta_{4}+\ddot{D}_{2}+g \eta_{4}\right)-\frac{l}{h} \ddot{D}_{3}>\frac{l}{h} g
$$

where $\ddot{D}_{3}$ is vertical acceleration, $\ddot{D}_{2}$ is lateral acceleration, $g$ is acceleration due to gravity, $\eta_{4}$ is instantaneous ship roll angle, $\ddot{\eta}_{4}$ is instantaneous ship roll acceleration, $h$ is the height of the centre of gravity of the subject from the deck, $l$ is half of the base of support length, and $\frac{l}{h}$ is the theoretical tipping coefficient. Since in this model the human body is modelled as a single rigid body, it cannot quantify the full human postural response, therefore it requires empirical tuning to work properly. In this approach, Graham developed the first formulation of a simple rigid body model for biomechanical quantification of motion severity in human balance. This model was a very simple approach based on considering the human body as a rigid block with mass, mass moment of inertia and simple geometrical characteristics [6,36].

\section{ii. Development of Simple Inverted Pendulum Model}

Laboratory postural studies suggest that dynamic postural stability models offer potential for more accurate description of the postural response in ship environments. In 2003, Wedge and Langlois [37] proposed a three-dimensional inverted pendulum model. Control strategies were employed by this model both in the sagittal and coronal planes based on joint angular position as well as velocity. A simple inverted pendulum model is a very simplistic approach to represent human postural stability during standing. The inter-relation between centre of pressure $(\mathrm{CoP})$ and centre of mass (CoM) provides the basis for postural control for this model [37]. CoP is defined as point of application of ground reaction forces (GRF) under the feet and $\mathrm{CoM}$ is defined as an imaginary point 
in the body or system of bodies at which whole body mass is assumed to be concentrated [44]. Projection of the CoM in the vertical direction (i.e., towards the ground) is called the CoG. Variable CoP-CoM (difference in centre of pressure and centre of mass) is defined as the "error" signal for the postural control mechanism of the model.

\subsection{Three-dimensional (3-D) Models for Pos- tural Stability Analysis}

Most postural stability models developed so far apply either in the sagittal plane or the coronal plane. However two-dimensional models are not the best option to describe the complex nature of human postural stability and to simulate different balancing strategies. Levin and Mizrahi (1995) proposed a threedimensional, five-joint, five-segment humanoid model in order to quantify CoM strategy and to describe postural sway dynamics. Later, in 2008, a two-link three-dimensional inverted pendulum was developed in order to describe human body dynamics [38]. In this model, a two-rigid-link strategy was used which bisected the human body into two parts: upper body and lower extremity. Both ankle and hip strategies were outlined in this modelling. Also, lower extremities (legs) were allowed to move only in the sagittal plane while upper extremities were also allowed to undergo rotation in the coronal plane, which was activated by hip torque. Transversal rotation between the two links was not

allowed. Spatial inverted pendulum model is regarded as one of the prominent three-dimensional models in postural stability studies.

\section{i. Spatial Inverted Pendulum Model}

Research to date, has concluded that simple inverted pendulum models play 


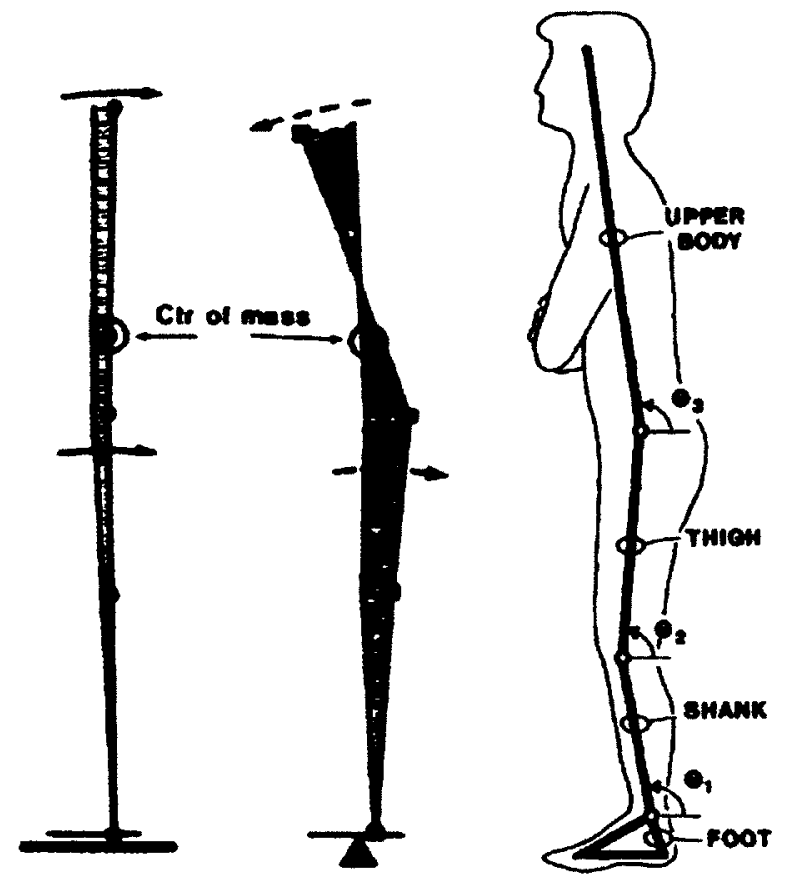

Figure 2.6: (a) Single and double inverted pendulum for demonstration of ankle and hip postural control strategies [47] (b) Four-segment inverted pendulum model [14].

an important role in investigating maintenance of postural stability in various circumstances including ship motion conditions. Referring to Fig. 2.6, it is clear that the number of articulated joints which are required for particular inverted pendulum models are dependent on the range of joint motion for each particular joint, environmental conditions, and postural stability task [38]. In this way, it can be demonstrated from Fig. 2.6 (a), that ankle and hip strategies are represented by a single segment and two-segment representations, respectively [38].

A spatial inverted pendulum approach can be used to represent shipboard tasks in a simple proper manner. In Fig. 2.7, point A represents the location of the interface between the ship and the inverted pendulum while point $B$ refers to $\mathrm{CoM}$ of the articulated segment. Two generalized co-ordinates $\theta_{i}$ and $\theta_{j}$ track 


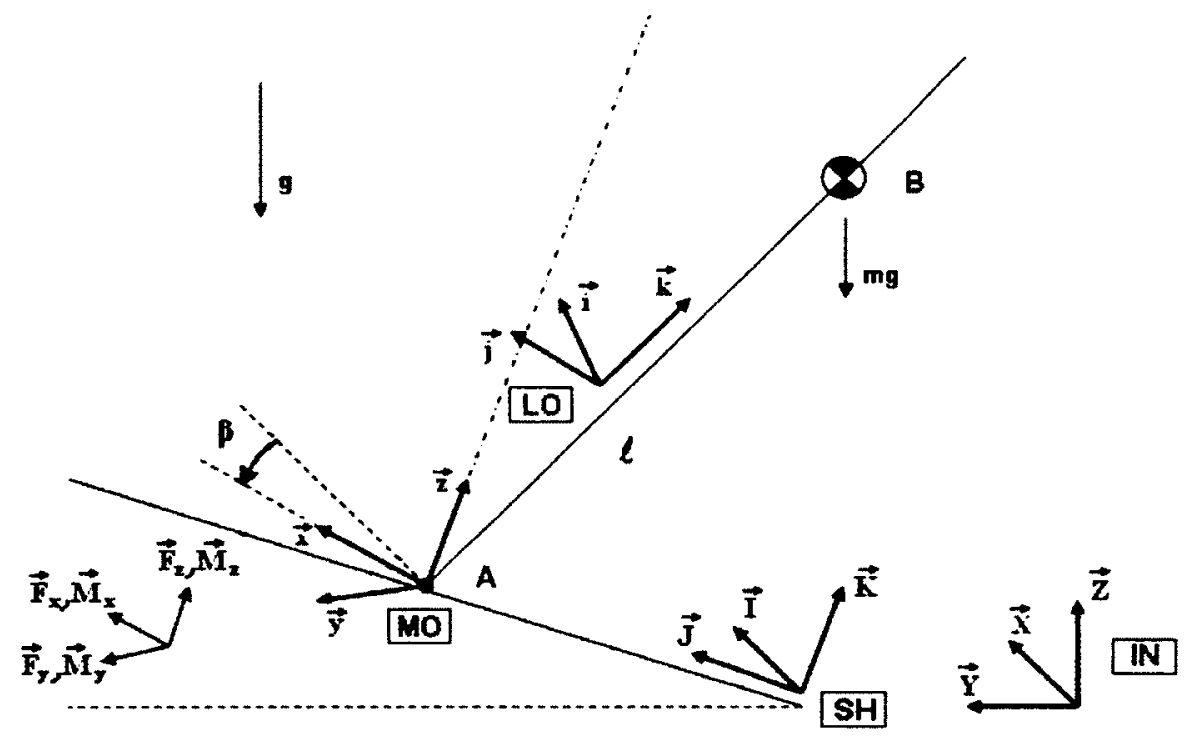

Figure 2.7: Schematic diagram of a single link spatial inverted pendulum [38].

the rigid body segment articulation (which correspond to sequential roll and pitch motion of an articulated rigid body segment relative to the ship deck). Four different coordinate systems are defined for this model. IN represents the inertial coordinate system, SH represents the ship coordinate system, MO is model coordinate system, and LO represents the local coordinate system attached to the body segment. Interface forces and moments can be tracked by $F_{x}, F_{y}, F_{z}$ and $M_{x}, M_{y}, M_{z}$ respectively [38]. The implemented model was verified for a single articulated segment and two degrees of freedom for postural stability analysis. A framework has been designed to extend this model to multiple articulated segments with a multi-degree of freedom postural stability modelling approach [38].

\subsection{State of the Art}

This section outlines the most recent developments in technology that is used in the field of postural stability investigation to capture motion data: 


\subsubsection{A Six-degree of Freedom (6-DOF) Human Motion Tracking System using Miniaturized Inertial Sensors}

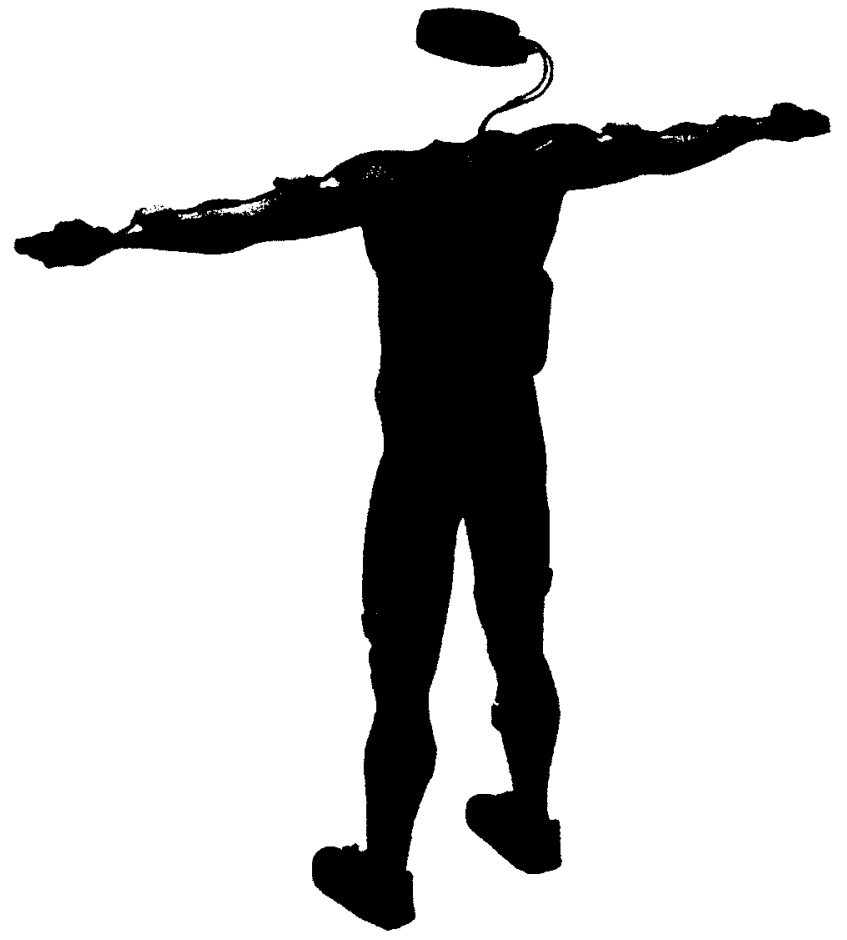

Figure 2.8: Xsens MVN with 17 sensor modules [43].

This is an advanced method of optical data collection technology using an Xsens MVN motion capture suit, which does not need external cameras, emitters, or any kind of markers (Fig. 2.8). The MVN sensor-suit is completely ambulatory comprising of 17 MTx body-worn sensors with two Xbus Masters. Xbus Masters observe as well as synchronize all sensor sampling, provide power to sensors, and handle wireless communication to transmit data to a laptop or $\mathrm{PC}$, where data processing takes place. Moreover, the MVN studio can be used as a graphical user interface (GUI) for analysis of motion data from a real time window as well as from previous recordings [43]. Also, the MVN studio SDK is capable of processing real-time 3D position aiding inputs, allowing real-time character animation. Thus, the system can be used for both indoor 
and outdoor measurements, since there is no restriction on data collection due to missing markers or lighting. This sensor suit is capable of capturing motion data while performing any activity: ruming, jumping, crawling, or walking [43]. However over time, inertial sensor data integration leads to drift errors occurence due to presence of sensor noise, sensor signal offset or orientation problem.

\subsubsection{Low-cost Motion Capture System for Better Postural Stability Analysis during Onboard Ship Tasks}
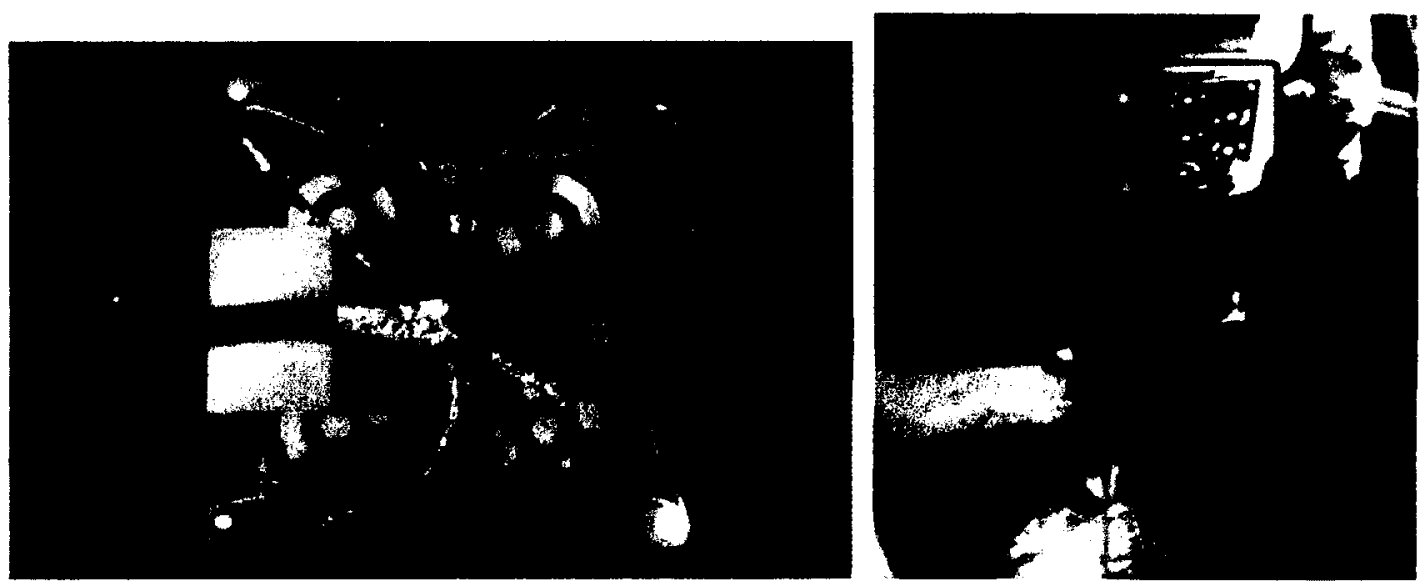

Figure 2.9: (a) Videogrammetric plate of MTx sensors embedded with four coloured high power LEDs for onboard ship tasks; (b) Attachment of videogrammetric plate unit at centre of mass height and MTi-G sensors for measurement of ship motion [40].

A wide range of experimental techniques are available for postural stability analysis of onboard ship tasks. Recently, a new methodology has been proposed for motion data acquisition of such tasks, which comprises two main equipment modules (a) videogrammetry; and (b) an inertial measurement unit (IMU) for motion measurement (Fig. 2.9). The videogrammetry system corresponds to a video camera arrangement which operates autonomously and stores the data collected in motion 
environments on internal memory. These cancorders have $1920 \times 1080$ pixel resolution with complementary metal oxide semiconductor (CMOS) embedded with an interlaced mode in order to get better effectiveness of the video output signal [40]. For experimental data collection during onboard typically, three video cameras are used, where two cameras are mounted to view simultaneously an individual facing forward. The position of third camera is secured so that it could observe and record the subject in the lateral direction. Using inertial measurement unit methodology, test subjects are equipped with MTx sensors positioned at their CoM (on the front side). The MTx sensors are secured with a photogrammetric plate which makes tracking of data possible via a built-in algorithm. Four high-powered coloured LEDs are arranged at the corners of the force plate and identified as 'colour-coded' three-dimensional markers. An MTi-G sensor is projected vertically onto the participant's CoM height on the cabin deck [40]. There is also a GPS antenna that must be mounted on top of the Pilots Bridge (platform from where ship is commanded). A common PC is used to collect data obtained from both sensors with an acquisition rate of $120 \mathrm{~Hz}$. This arrangement reduces hardware complexity onboard during experimentation, and therefore is expected to simplify data collection in the field of shipboard postural stability research [40].

\subsubsection{SimMechanics Technology in Simulation of Human Posture (Advancement in Multibody Dynamic Model Development)}

SimMechanics provides the possibility of modelling three-dimensional mechanical systems within the Simulink environment. Rather than deriving and programming lengthy equations and codes, the multibody simulation tool can be used for multibody dynamic model development which is composed of bodies, joints, constraints, 
and force elements [42]. The associated three-dimensional animation allows visualization of system dynamics. SimMechanics is suitable for studying loads imposed on joints, which is a major factor of postural stability analysis. Moreover, SimMechanics with other MATLAB toolboxes provides a wide variety of applications in medical research, development of rehabilitation equipment, and control systems for identification of physiological disorders. Therefore, a human body model animated via SimMechanics is a modifiable approach where the weight and moment of inertia of the whole human body or segments of it can be changed, allowing calculation of the resulting forces and moments in joints in very comprehensive way [42].

\subsection{Summary}

Research into postural stability in motion environments is of ongoing interest. Single link biomechanical models have been developed into multi-link biomechanical models of humanoids with implementation of simulated sensory organs and muscle actuation systems as the experimental techniques and computational technologies have advanced. Initially, these models were developed with the objective of basic understanding of human physiology and postural stability strategies, while they were also used to study the effects of as well as the risks associated with motion environments during various operational activities. Laboratory experiments have also been conducted in order to understand the effects of severe deck motion on naval crew members performance at ship tasks. Despite progress to date, there are still many issues related to postural stability maintenance during shipboard activities that need to be resolved. On the other hand there exists substantial literature on postural stability research that is ready to be applied to quantify effects of motion environments beyond ship deck tasks. 


\section{Chapter 3}

\section{Experimental Set-Up}

\subsection{Introduction}

The basic objective of the experimental investigation was to determine human postural stability characteristics for different levels of motion severity. This was achieved by measuring joint motions of individual subjects while they were subjected to simulated ship motion. This provides basic information about each joint's contribution to maintaining a stable postural state during changing motion environments. Moreover, the mechanical work performed by each joint can be determined to provide a measure of the total mechanical work performed by the human body in order to maintain its balanced state. This is considered important information for characterizing human postural strategies in a quantitative manner. In addition, the mechanical work performed by different joints is correlated to metabolic energy expenditure data that was collected to provide a measure of motion induced fatigue (MIF) and similar disorders that are responsible for gradual degradation of crewmember's and ship operator's abilities during maritime operational activities. 


\subsection{Modules for Experimental Set-Up}

The experimental set-up basically comprised five basic modules. These modules were:

- MOOG-6DOF stewart platform motion simulator;

- Opti-track motion capture system;

- Tek-scan insole sensor system for transient foot pressure data;

- BB1LP respirometry system; and

- Load cell with force plate.

\subsubsection{MOOG-6DOF Stewart Platform Motion Simulator}

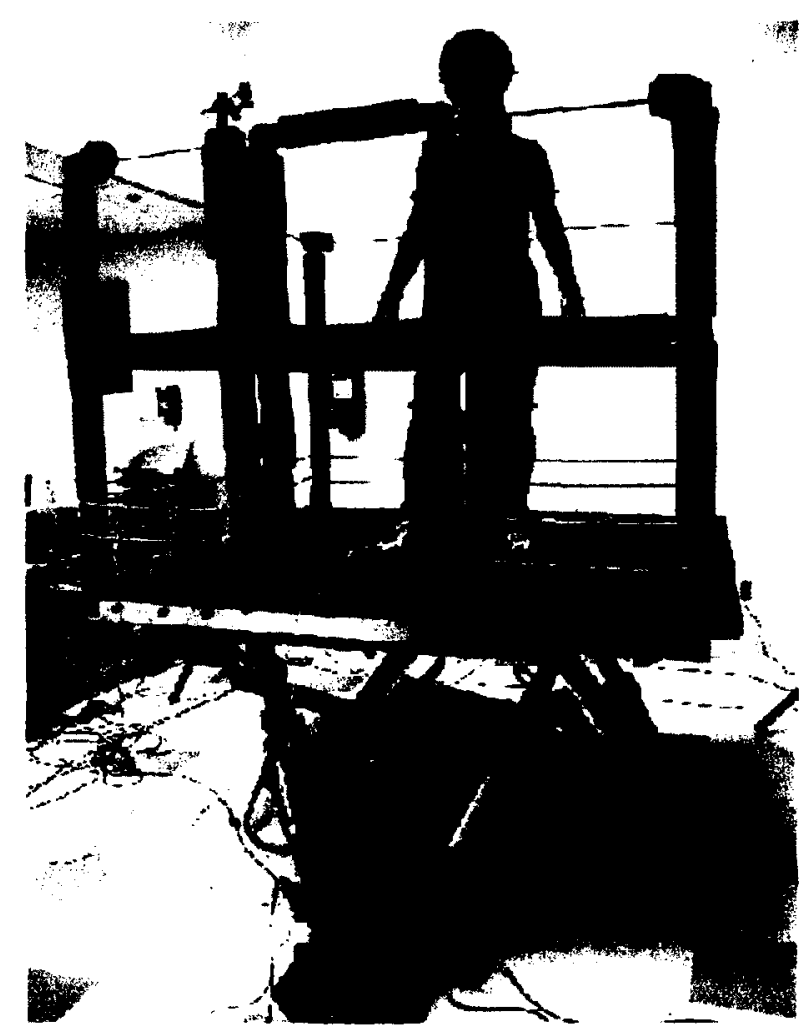

Figure 3.1: Experimental set-up with MOOG Stewart platform, railings, and padding. 
This motion simulator or motion platform was used to simulate ship motion typical of various sea states (refer to Fig. 3.1). A stewart platform is a parallel robot which is supported by six actuators mounted in pairs at its base. This platform can provide six-degrees of freedom: three translational motions (lateral, longitudinal, and vertical) as well as three rotational motions (roll, pitch, and yaw) [49]. Fig. 3.2 demonstrates the hardware set-up required for rumning motion profiles for data collection. The MOOG-6DOF platform movements are controlled by inter-communication of two computer set-ups: motion platform computer and applied dynamics computer. Once the motion platform computer is turned on by switching on the power switch and providing the required information, it needs host communication in order to provide commands to the motion platform. Host communication is provided through the

applied dynamics computer, where the ethernet host software is running. Provided communication is maintained between the motion platform and the applied dynamics computer. Motion profiles can be selected and various control commands such as base parking, engaging, and returning to the home position can be given to the motion simulator through the ethernet host software.

\section{Generation of Motion Profiles for the Motion Simulator}

Power spectral density (PSD) representations can be used to quantify the characteristics of any random variable in the frequency domain. From the PSDs, pseudo-random time series of any random vibration can be generated. Based on this theory, representative ship motion time histories for each of the six ship degrees of freedom can be obtained from ship's response amplitude operators (RAOs) [50]. The RAOs were calculated by a standardized computer programme and are directly related to transfer functions of ship motions to wave inputs. The continuous frequency spectra of the ship's motions were converted into a finite number of discrete components for approximation of the RAOs. Moreover, RAOs are combined with a selected seaway 

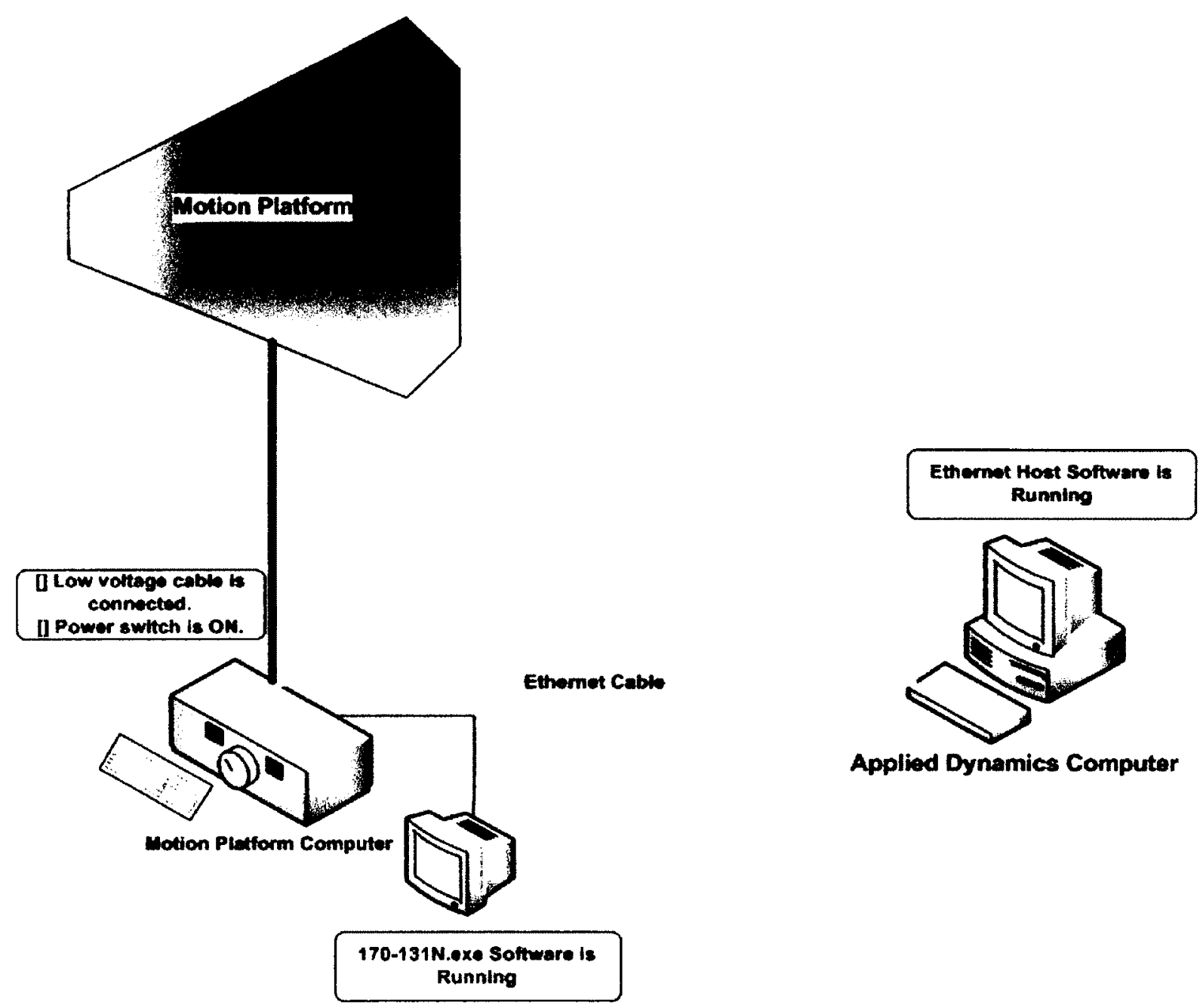

Figure 3.2: Hardware set-up of MOOG-6DOF motion platform prior to running motion profiles.

spectrum in order to generate the discrete PSD of each degree of freedom of the ship motion in that particular seaway [50]. Consequently, a time series as given by Eqn. (3) was generated by considering discrete components and summing them to represent a continuous ship motion as follows, 


$$
q_{i}(t)=\sum_{j=1}^{M} A_{i j} \sin \left(\omega_{i j} t+\theta_{i j}+\theta_{R N D j}\right)
$$

here $q_{i}(t)$ represents a specific motion (e.g., roll, pitch, etc.), $t$ is time, $M$ is the number of components used for approximation of the continuous spectrum, $A_{i j}$ and $\theta_{i j}$ are corresponding amplitude and phase responses of the PSD, and $\theta_{R N D j}$ is a random phase angle which represents randomness of the sea surface.

In our experiment, Eqn. (3) was used to generate various motion profiles each representing a different motion at various sea states. Twelve such motion profiles were generated for the MOOG-6DOF simulator. Using North Atlantic Treaty Organization (NATO) sea state scale standards, motion profiles were generated in increasing severity from low wave height, i.e., 1 meter waves to medium waves (with 2 meter height) and 5 meter and then to severe state with 7 meter wave height. Three types of motions (i.e., roll (R), pitch (P), and general/combined ship motion with pitchroll-yaw (PRY) factor) were considered. Each of the three motion types possesed 4 motion profiles categorized on the basis of wave height, which represented 4 different sea states. It should be noted that in this thesis sea states 1 through 4 refer merely to four different levels (as shown in the Table 3.1) and must not be confused with the NATO scale of standard sea conditions that uses the term "sea state" to refer to specific ranges of wave elevation. The motion profiles are allowed to run in a random order for a specified time period of 360 seconds while human test subjects are requested to maintain balance during the motion. Table 3.1 depicts the 12 motion profiles used to run the experiment.

Table 3.2 shows the minimum, maximum and RMS values for all twelve motion cases in $\mathrm{x}, \mathrm{y}$, and $\mathrm{z}$ directions. It shows the severity order for pitch, roll and combined motion in different motion states. 
Table 3.1: Motion profiles

\begin{tabular}{|l|c|c|c|c|c|c|}
\hline Motion Profile & Sea State & Wave Height $(\mathrm{m})$ & Ship Heading(deg) & Pitch & Roll & Yaw \\
\hline 1000 & 1 & 1 & 000 & yes & no & no \\
2000 & 2 & 2 & 000 & yes & no & no \\
5000 & 3 & 5 & 000 & yes & no & no \\
7000 & 4 & 7 & 000 & yes & no & no \\
1045 & 1 & 1 & 045 & yes & yes & yes \\
2045 & 2 & 2 & 045 & yes & yes & yes \\
5045 & 3 & 5 & 045 & yes & yes & yes \\
7045 & 4 & 7 & 045 & yes & yes & yes \\
1090 & 1 & 1 & 090 & no & yes & no \\
2090 & 2 & 2 & 090 & no & yes & no \\
5090 & 3 & 5 & 090 & no & yes & no \\
7090 & 4 & 7 & 090 & no & yes & no \\
\hline \hline
\end{tabular}

The motion platform was equipped with a $2 \times 2$ meter wooden base to accommodate a force plate that is discussed later. An upper frame with railings was used in order to provide safety to human test subjects in the case of loss of balance. The wooden base of the platform was also covered with high friction tiles in order to minimize slipping possibilities and enhancing safety during experimentation. 
Table 3.2: Minimum, maximum and root mean square values for twelve motion profiles

\begin{tabular}{ccccccccccc}
\hline \hline Motion Profile & Min $_{x}$ & Min $_{y}$ & Min $_{z}$ & $M_{a x}$ & $M a x_{y}$ & $M a x_{z}$ & $R M S_{x}$ & $R M S_{y}$ & $R M S_{z}$ \\
& radians & radians & radians & radians & radians & radians & radians & radians & radians \\
\hline 1000 & 0.000 & -0.017 & 0.000 & 0.000 & 0.010 & 0.000 & 0.000 & 0.003 & 0.000 \\
2000 & 0.000 & -0.030 & 0.000 & 0.000 & 0.029 & 0.000 & 0.000 & 0.008 & 0.000 \\
5000 & 0.000 & -0.096 & 0.000 & 0.000 & 0.105 & 0.000 & 0.000 & 0.026 & 0.000 \\
7000 & -0.273 & -0.004 & -0.006 & 0.274 & 0.003 & 0.006 & 0.094 & 0.001 & 0.002 \\
1045 & -0.023 & -0.012 & -0.003 & 0.024 & 0.012 & 0.003 & 0.009 & 0.004 & 0.001 \\
2045 & -0.064 & -0.025 & -0.007 & 0.067 & 0.027 & 0.007 & 0.062 & 0.010 & 0.003 \\
5045 & -0.211 & -0.069 & -0.027 & 0.262 & 0.063 & 0.028 & 0.081 & 0.025 & 0.009 \\
7045 & -0.273 & -0.004 & -0.006 & 0.274 & 0.003 & 0.006 & 0.094 & 0.001 & 0.002 \\
1090 & -0.054 & -0.001 & -0.001 & 0.059 & 0.001 & 0.002 & 0.019 & 0.0005 & 0.0006 \\
2090 & -0.121 & -0.002 & -0.003 & 0.109 & 0.002 & 0.003 & 0.414 & 0.0008 & 0.001 \\
5090 & -0.273 & -0.004 & -0.006 & 0.275 & 0.003 & 0.006 & 0.094 & 0.001 & 0.002 \\
7090 & -0.273 & -0.004 & -0.006 & 0.275 & 0.003 & 0.006 & 0.094 & 0.001 & 0.002 \\
\hline
\end{tabular}




\subsubsection{Opti-track Motion Capture System}
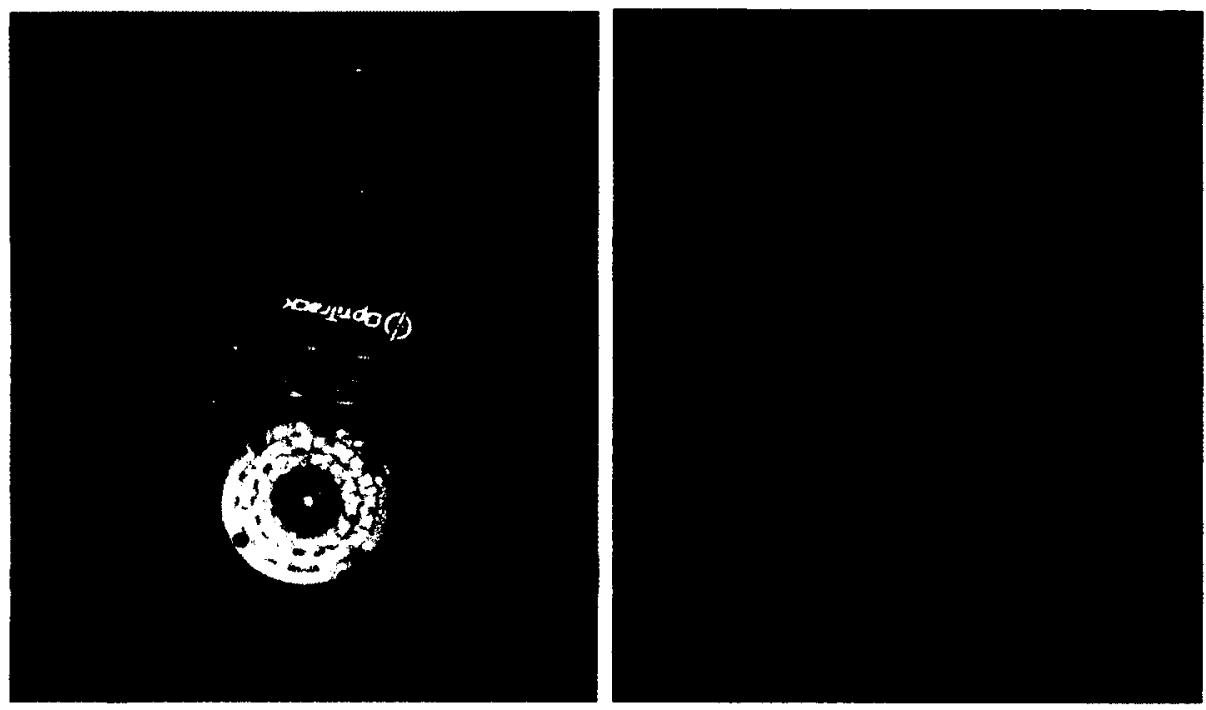

Figure 3.3: (a) Opti-track IR camera [51]; (b) Retro-reflective markers [51].

An Opti-track system is used to collect motion data on human subjects while maintaining stability during ship motion. This set up is comprised of 8 infra-red LED cameras which were arranged so that they could capture the body posture of the human subjects in their efforts to maintain their balance (Fig. 3.3a). This was accomplished by a motion capture suit with 34 retro-reflective markers (Fig. $3.3 b$ ) that was worn by the human participants prior to data collection. The locations of markers on the motion capture suit were defined in an appropriate manner so that all body segments could be detected by the cameras properly [51]. Markers were placed at locations on the body where the skin is close to the skeleton in order to reduce skin sliding as much as possible. The collective set up of LED arrays of cameras and markers on body segments determined the anatomical reference points of the body. For effective operation of the Opti-track setup, all reflective objects within view of capture area were removed and lights were dimmed. The Opti-track system is capable of capturing motion data at $100 \mathrm{~Hz}$ frequency, and provides sub-millimeter 
( $\mathrm{mm}$ ) accuracy when set-up is effectively done by selecting capturing frequency of 100 frames per seconds.

\section{Calibration of the Opti-track System}

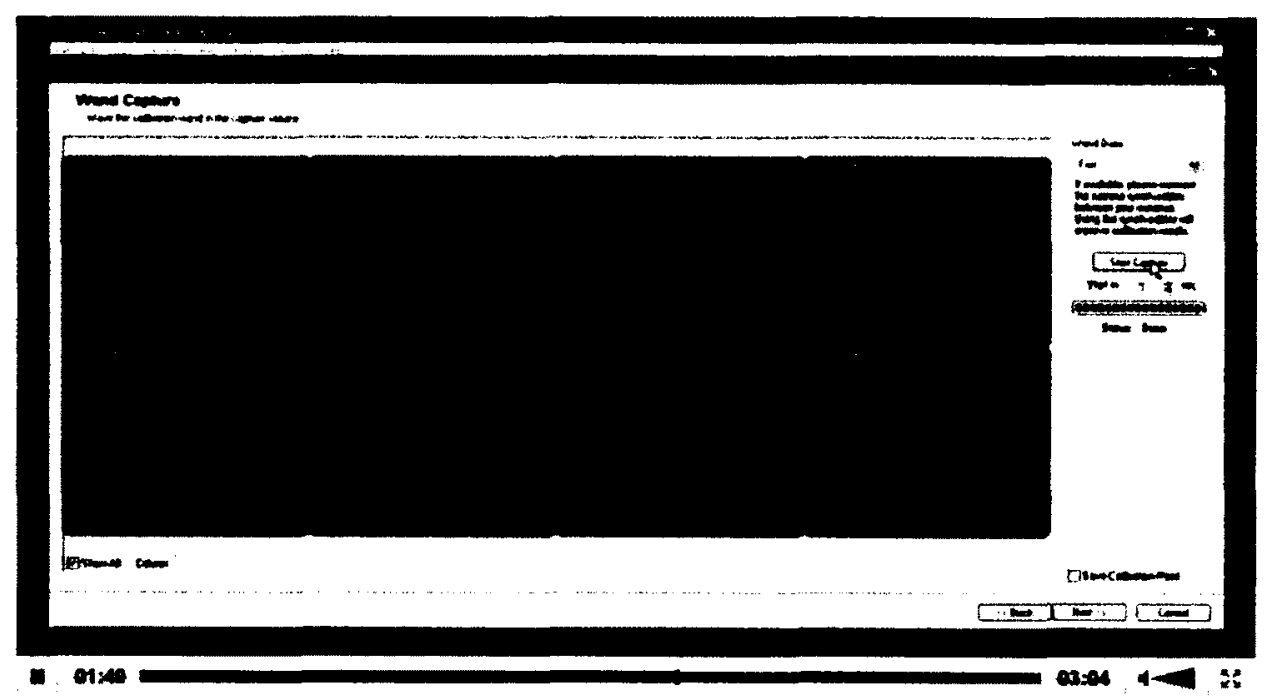

Figure 3.4: Wand capture using 8 infra-red camera system.

For calibration of the Opti-track motion capture system, the motion platform was raised to its neutral position by engaging it from its parked state. Calibration included two basic steps: (i) a wanding procedure was used to calibrate the cameras for better capturing accuracy and an origin point on the ground plane was defined which behaves as the reference point; and (ii) a virtual skeleton was fit to the human subject.

Wanding was required to calibrate and assess the quality rating of each of the eight infra-red cameras. A maximum of one or two cameras may be considered below the highest level (i.e., excellent quality) to ensure high-qualtiy data collection. The wanding procedure was carried out throughout the platform capture area by swaying a wand while making sure that each camera was able to capture the wand motion (Fig. 3.4) [51]. After the wanding procedure, ground plane was set using calibration 
square for determination of origin as well as direction of $\mathrm{x}, \mathrm{y}$, and $\mathrm{z}$-axes. The second step of the calibration involved assigning a virtual skeleton to the human subject according to the outlines described by the Arena software. In this step, the human subjects wore a black form fitting Velcro suit with 34 retro-reflective markers attached to it, which fully represent 15 segments of the human body in 3-D space (Fig. $3.5 a$ and $b)$.
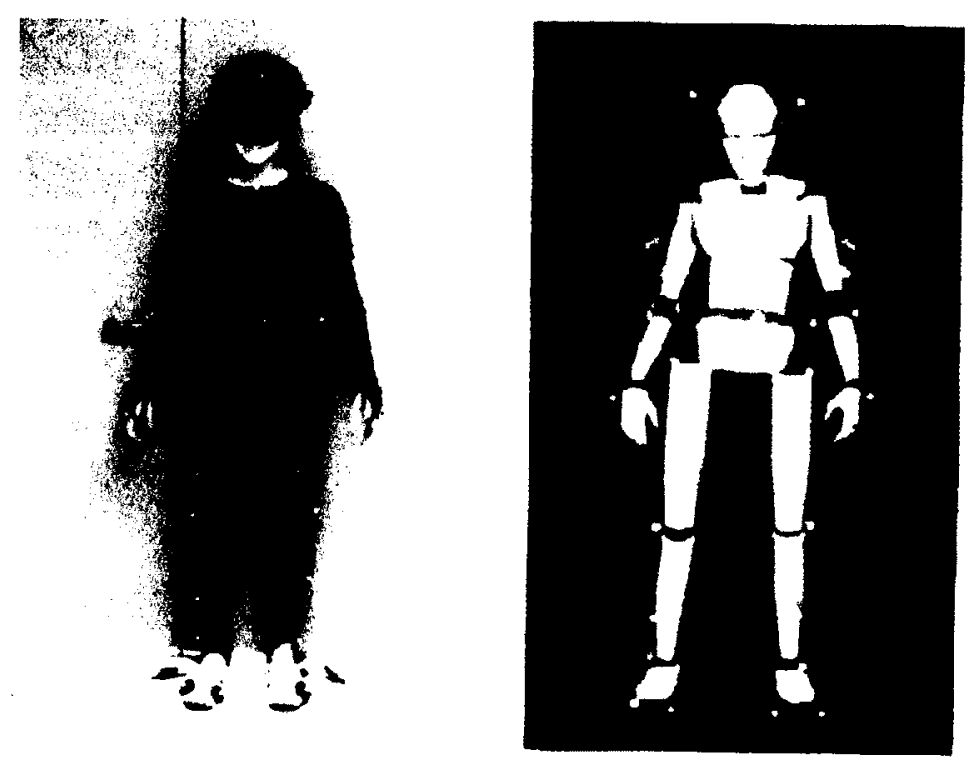

Figure 3.5: (a) Human subject in motion capture suit; and (b) Opti-track 15 segment skeleton [51].

The T-Pose data of the human subjects prior to actual data collection was captured. T-pose is the standing position taken by the subjects with arms elevated to shoulder height and legs seperated equal to shoulder width. This was necessary to ensure that all 34 markers were properly reconstructed in the 3-D image of the cameras as well as to ensure effectiveness of camera capturing. The first 15 seconds of a total 30 second data collection was utilized for setting the T-pose of the human subject. This was followed by a second period of 15 seconds of data collection while the subject was in motion. The collected data was trajectorized after recording in order to save 
the captured session in .pt3 file format by providing binding of the skeleton and rigid body $3-\mathrm{D}$ motion to a point cloud. This trajectorised data was finally available in any of .C3D, .BVH or .FBX format for further analysis [51].

\subsubsection{Tek Scan Insole Foot Pressure Sensor System}

Foot pressure sensors of the Tek Scan system were used to collect time-varying foot pressure data. This information was collected as numerical values of the pressure applied at sensors showing pressure intensity as well as coloured graphical images of time-varying pressure contours.

\section{Description of Foot Pressure Sensors for Data Measurement}

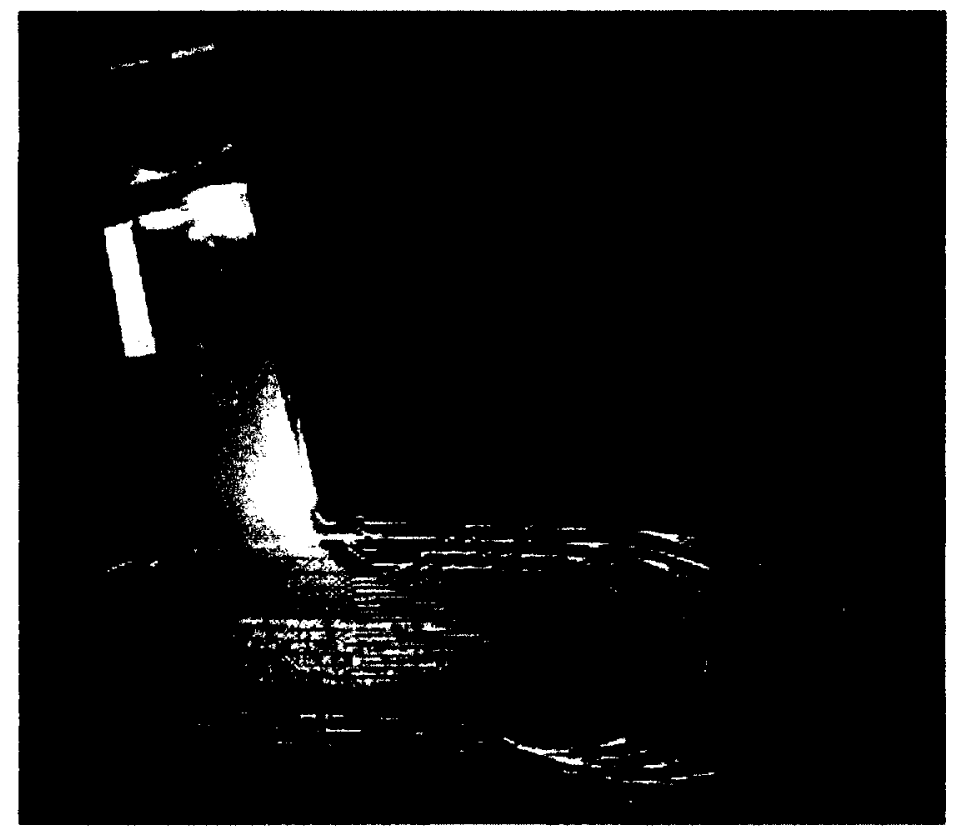

Figure 3.6: Insole with 960 sensels for foot pressure data measurement.

The foot pressure sensor system comprises ultra-thin, double-layered sheets of $0.18 \mathrm{~mm}$ thickness which are further thickened with a protective backing. The composition of foot pressure insoles includes pressure sensitive, conductive, and resistive 
silver-based links, arranged in 60 columns and 21 rows and embedded in Mylar coating (Fig. 3.6). These were fit into subject's shoes by cutting them according to the subject's foot size [52]. The intersection of rows and columns lead to a pressure sensing "cell" construction, each called a sensal with 960 in each insole [52]. The resistance of each cell is inversely proportional to the pressure applied at the surface of cell. When load is applied to the sheet, there is change in resistance of the pressure sensitive resistive link, and electrical output from system is generated accordingly. In this way, with movement, as pressure on surface changes, the resistance also changes proportionally. This information can be collected from the cells and relayed through the embedded wiring to a pre-amplifier, where analog to digital data conversion takes place. Further, finally this data is transmitted to the central processing unit (CPU) of a data collection computer resulting in recording and storing of cell pressures in computer memory [52]. Tek scan software provides the capability to visualize both two-dimensional and three-dimensional data in the form of 2-D or 3-D contours. It is also possible to convert foot pressure data into American Standard Code for Information and Interchange (ASCII), which can be further available for other statistical analysis using other software such as MATLAB.

\section{Equilibration and Calibration of Tek Scan Pressure Sensor System}

\section{i. Equilibration}

There are 960 sensels located at each intersection of rows and columns in each insole. Each sensor is unique from every other sensor in its response to applied pressure [53]. Hence, it is necessary to equilibrate all sensors, to compensate for slight variations among them [53]. To this end, a special piece of equipment is used for equilibration/calibration of Tek Scan system. This is a thin flexible membrane pressurized against sensors. Participants are instructed to apply uniform pressure for all the foot pressure sensors. Once the equilibration is 
done and the "equilibration" procedure is complete, a graphical representation of the real time pressure distribution appears [53]. Therefore, the equilibration procedure ensures the consistency between all the sensors.

\section{ii. Calibration}

Calibration is required to convert the digital outputs into actual pressure units (mmHg/PSI). Calibration was achieved by asking subjects to stand on one foot at a time such that the integrated pressure can be calibrated against the subject weight as measured using a spring scale [53]. Subjects were directed to keep shifting the weight on the foot to be calibrated for at least 5-10 seconds, repeating the procedure for both feet. Calibration results for both feet was saved properly once it was complete.

\section{Data analysis using Tek Scan Foot Pressure Sensor System}

Due to the thin semiconductor coating which is applied between the conductive rows and columns, electrical resistance is inversly proportional to the applied pressure. The matrix of sensels results in an output in the form of a matrix of voltage changes, which can be displayed on the computer with different colors to distinguish different pressure levels. The colour range varies between blue and red (1-125 psi) through green, yellow, and orange. Therefore, high and low pressure areas are evident as the variation in pressure takes place from heel to toe over the stance period during movement on the motion platform [52].

\subsubsection{BB1LP Respirometry System}

A metabolic analyzer is specially designed to measure the amount of metabolic energy expended by an individual in terms of calories burnt by the subject's body [55]. The 


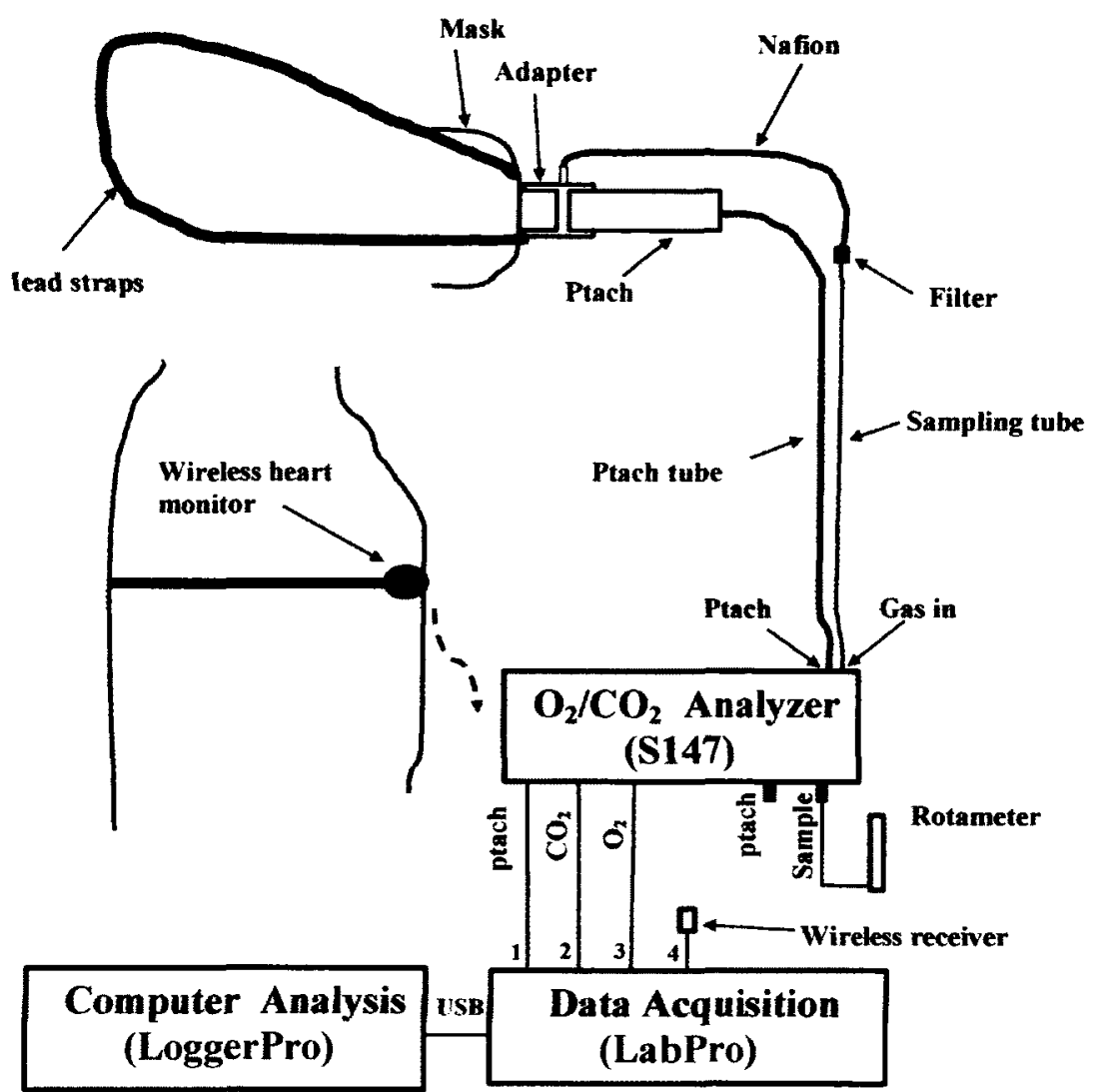

Figure 3.7: BB1LP respirometry system [55].

BB1LP respirometry system is specially designed for this objective. Fig. 3.7 demonstrates the basic set-up of the BB1LP respirometry system for energy expenditure measurement.

\section{Components of the BB1LP Metabolic Analyzer}

The BB1LP Analyzer basically comprised of 5 main components.

i. $\mathrm{S} 147 \mathrm{O}_{2} / \mathrm{CO}_{2}$ Analyzer

As indicated in Fig. 3.7, the analyzer combines both a laser diode sensor for oxygen $\left(\mathrm{O}_{2}\right)$ detection and an infrared (IR) sensor for carbon dioxide $\left(\mathrm{CO}_{2}\right)$ 
detection [55].

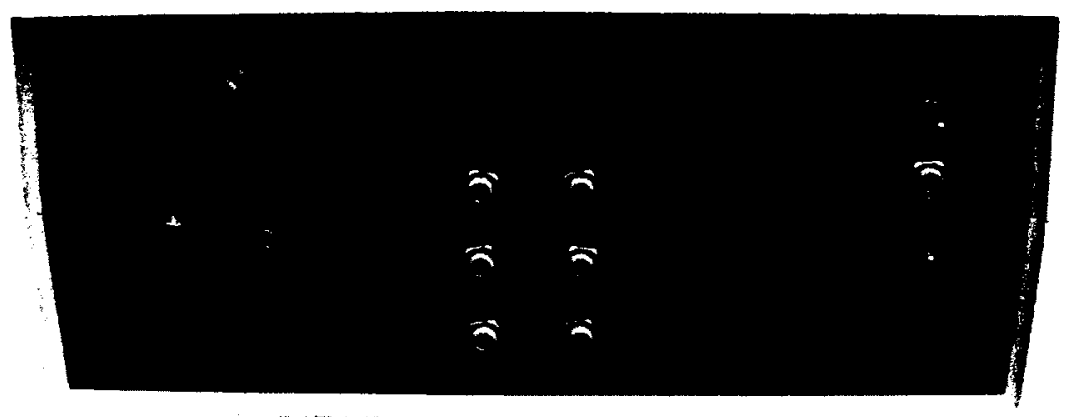

Figure 3.8: Front panel of $\mathrm{S} 147 \mathrm{O}_{2} / \mathrm{CO}_{2}$ analyzer [55].

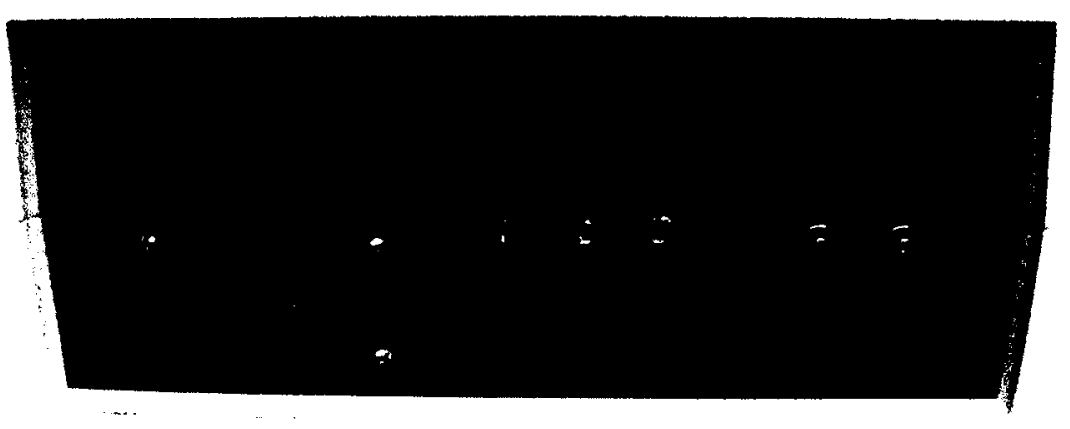

Figure 3.9: Back panel of $\mathrm{S} 147 \mathrm{O}_{2} / \mathrm{CO}_{2}$ analyzer [55].

The IR $\mathrm{CO}_{2}$ sensor works on the principle of absorption of $\mathrm{CO}_{2}$ by infrared light. An IR source is directed through the tube which contains the gas for analysis. The amount of IR light absorbed by the gas at a particular wavelength in the flow tube is detected by the sensor. The output of the detector is proportional to the amount of IR light absorbed by the sensor, which gives a direct measure of the amount of $\mathrm{CO}_{2}$ present in the tube [55].

The laser $\mathrm{O}_{2}$ diode sensor works on the principle of radiation absorbed by oxygen. The wavelength of the laser diode is very close to the absorption line for $\mathrm{O}_{2}$. On the basis of this, the amount of absorbed $\mathrm{O}_{2}$ can be detected efficiently. Together, the S147 assembly possesses the subsampling pump unit, differential sensor, as well as oxygen and carbon dioxide sensors [55]. 


\section{ii. F500 Gas Flow Meter}

The F500 flow meter has a range of $40-500 \mathrm{ml} / \mathrm{min}$. The flow meter is located at the back panel of the $\mathrm{S} 147 \mathrm{O}_{2} / \mathrm{CO}_{2}$ sensor for measurement of gas flow rate, which should be approximately $300 \mathrm{ml} / \mathrm{min}$. Flow rate adjustments can be made with the help of a pump speed control available on the front panel of the $\mathrm{S} 147 \mathrm{O}_{2} / \mathrm{CO}_{2}$ analyzer [55].

\section{iii. Face Mask, Head Strap, and Pneumotachometer (Pneumotach)}

Face masks are air-cushioned units, which collect breath for analysis. They are placed in position over the mouth and nose and secured by head straps, which connect to four points on the mask. Moreover, a pneumotachometer (which detects gas flow) is attached to the mask using a Teflon adaptor. Precaution must be taken in mounting the adaptor, so that the Nafion tube (braided tube which connects to adapter between mask and pneumotachometer) attaches to either the top or the centre of the mask to prevent accumulation of saliva as well as condensation due to breathing [55].

\section{iv. Tubing and Connectors}

The $\mathrm{S} 147 \mathrm{O}_{2} / \mathrm{CO}_{2}$ analyzer is connected to the rest of the BB1LP system using assorted tubing and connectors. Basically, two joined tubes of length 1.5 meter each are used to connect the pneumotachometer to the front panel of the S147 analyzer unit [55]. Sample gas is carried to the "Gas in" port on the front of S147 analyzer via 1/16 inch tube with an air-filter and braided Nafion tube. In addition, the flow signal is carried by an $1 / 8$ inch tube from the end of the pneumotachometer to the "Ptach" which is on the front of S147 analyzer unit. Also, at the back of S147, the F500 flow meter is attached to a "sample vent", in order to measure sample gas flow [55]. 


\section{v. Heart Rate Monitor}

Detection of electrical signals generated by the heartbeat at the skin surface is carried out by heart rate sensor. These signals are measured by electrodes which are embedded in a chest belt. Transmission of the electrical signals takes place wirelessly from the chest belt to receiver, which should be placed within 1 meter of the chest belt for accurate signal detection [55]. Signal quality can be improved by providing better skin contact by applying saline solution to the two electrodes [55].

\section{Calibration of $\mathrm{O}_{2} / \mathrm{CO}_{2}$ Analyzer Sensors}

This calibration is required to ensure that both sensors are effectively measuring the $\mathrm{O}_{2}$ absorption and $\mathrm{CO}_{2}$ exhalation values. Calibration of the BB1LP respirometry system involvs two main adjustments: zero and span adjustment. For zero adjustments, the ambiant room air is needed with "zero" $\mathrm{CO}_{2}$ and water vapour removed. It can be checked by observing the Logger Pro meters, where $\mathrm{CO}_{2}$ is adjusted to $0 \%$ and $\mathrm{O}_{2}$ is adjusted to $20.95 \%$ by adjusting the "zero control" with a screwdriver on the front panel of the analyzer [55]. For span adjustment, a calibration gas mixture of $16 \% \mathrm{O}_{2}, 5 \% \mathrm{CO}_{2}$ and $79 \% \mathrm{~N}_{2}$ is used, regulated to outlet pressures of 2 psi to 5 psi and at a flow rate of $400 \mathrm{ml} /$ minute in order to maintain continuously equal flow of gas during the calibration process. Adjustment of $\mathrm{O}_{2}$ to $16 \%$ and $\mathrm{CO}_{2}$ to $5 \%$ can be made by adjusting the $\mathrm{O}_{2} / \mathrm{CO}_{2}$ "span control" of these sensors with a screwdriver from the S147 analyzer and checking it on the Logger Pro screen [55].

\section{Operation of BB1LP Respiratory System}

Breath analysis takes place when a subject breathes through the face mask. An adaptor connects face mask to pneumotach (ptach) flow meter. Breath flow can be measured on the basis of differential pressure between the inside and the outside of the 
pneumotach. Two pressures are conveyed to the $\mathrm{S} 147$ analyzer for differential pressure analysis. The pressure inside the pneumotach can be conveyed to the "ptach" input on the front side of the analyzer via a 1.5 meter long tube; while atmospheric pressure can be conveyed through the "ptach" vent on the back side of the S147 [55]. The flow rate of gas samples was maintained at approximately $300 \mathrm{ml} /$ minute. A tubing between the mask or ptach adaptor and "Gas in" on the front of S147 was used to sub-sample the inhaled and exhaled air at the known flow rate of $300 \mathrm{ml} /$ minute. Moreover, a 25 micron filter was also attached to "Gas in" tubing in order to prevent contamination of the optical gas sensors by filtering air particulate. In order to check the subsample flow, the rotameter is attached to the sample vent on the back side of the S147 analyzer. Analog outputs, i.e., flow (ptach), $\mathrm{CO}_{2}$, and $\mathrm{O}_{2}$ from the back side of the S147 are further conveyed to channels 1, 2, and 3 respectively, which are delivered to the LabPro data Logger [55].

The wireless heart rate monitor worn as a chest band had its receiver attached to channel 4 of the Labpro. Logger Pro is the software that was installed in the host computer to control collection of data in LabPro. Logger Pro was also programmed to plot air flow, $\mathrm{CO}_{2}$ production, and $\mathrm{O}_{2}$ consumption as a function of time. Metabolic energy used by the subject was calculated using Weir's Metabolic Energy equation (in $\mathrm{kcal} /$ day):

$$
\text { MetabolicEnergy }=\left(3.9 \times\left(\mathrm{VO}_{2}\right)+1.1 \times\left(\mathrm{VCO}_{2}\right) \times 1440\right)
$$

where $\mathrm{VO}_{2}$ represents the volume of oxygen consumed, $\mathrm{VCO}_{2}$ represents the volume of $\mathrm{CO}_{2}$ exhaled at standardized temperature and pressure conditions.

Logger Pro automatically evaluates the amount of metabolic energy expended (in $\mathrm{kcal} / \mathrm{day}$ ) by the subject while he/she is maintaining his/her stability during simulated ship motion. Further, this software was able to detect individual breathes 
as well and display various breath parameters. There is also in-built capability to save data for further analysis or import it to worksheets once data collection is complete [55].

\subsubsection{Load cell with Force Plate}

The load cell arrangement along with the force plate collectively was used to measure components of force and moment applied by the subject under one foot. This force is balanced by the ground reaction force (GRF) which is synchronized with kinematic data both temporally and spatially [56]. The main concern during external force data collection is spatial synchronization of kinematic data with GRF data. This was resolved by establishing the origin of the global coordinate system (GCS) of the Optitrack module relative to the origin of the force platform. Participants put their right foot on the load cell located on the platform to generate 6 signals each denoting one of the three forces: $F_{g r f_{x}}, F_{g r f_{y}}, F_{g r f_{z}}$ and three moments: $M_{g r f_{x}}, M_{g r f_{y}}, M_{g r f_{z}}[56]$. These forces and moments were subsequently used in the equations describing the multi-body dynamics model to evaluate kinematic parameters. In addition to this, GRF components were used to validate the equations of the developed multi-body dynamic model (Chapter 5). 


\section{Chapter 4}

\section{Data Collection and Post-Processing}

\subsection{Introduction}

This chapter covers the procedures used for selection of subjects and data acquisition. In addition, data sampling and post-processing techniques are briefly discussed.

\subsection{Test Subject Selection}

Approval for this experiment was provided by the Carleton University Research Ethics Board (REB). All subjects were selected on the basis of their eligibility to meet the range of standards approved by the REB. Table 4.1 outlines the key physiological characteristics of all human subjects who volunteered for this study. Ten volunteers (6 male and 4 female) of varying physiological characteristics were selected as subjects for experimental investigation. All subjects had either no or very minimal experience on seagoing vessels. None of them suffered from any musculoskeletal disease, breathing problems, or balance disorders. All subjects were non-smokers. 
Table 4.1: Test subjects key characteristics

\begin{tabular}{|l|c|c|c|c|}
\hline Sub. No. & Sex & Age (yrs.) & Body Height (meter) & Body Mass (kg) \\
\hline 1. & Male & 23 & 1.70 & 56.69 \\
2. & Male & 25 & 1.67 & 72.57 \\
3. & Male & 26 & 1.72 & 58.05 \\
4. & Male & 27 & 1.67 & 65.05 \\
5. & Male & 22 & 1.61 & 68.03 \\
6. & Male & 27 & 1.80 & 83.33 \\
7. & Female & 20 & 1.60 & 50.69 \\
8. & Female & 25 & 1.65 & 72.20 \\
9. & Female & 25 & 1.65 & 68.33 \\
10. & Female & 24 & 1.60 & 58.05 \\
\hline \hline
\end{tabular}

\subsection{Data Acquisition}

Since all subjects were volunteers, data capture session scheduling was based on the subject's availability. Participants were asked to complete an inclusion-exclusion criteria form and sign the informed consent form after reading the experimental procedure and safety as well as security measures documentation.

Prior to starting the data collection process, the Opti-track cameras were calibrated to ensure that no errors would occur due to camera positioning during motion capture sessions. The subject was fitted with the appropriately-sized motion data capture suit, and 34 retro-reflective markers were placed at pre-determined locations on the body to represent the 15 segments of the body in 3-D space. Next, the subjects were fitted with foot pressure sensing insoles in their shoes. Insoles were trimmed according to subject's foot size for better data acquisition. The calibrated BB1LP respirometry system was connected to the subject by attaching the mask onto the participant and tightening the head straps. Fig. 4.1 represents the data collection 


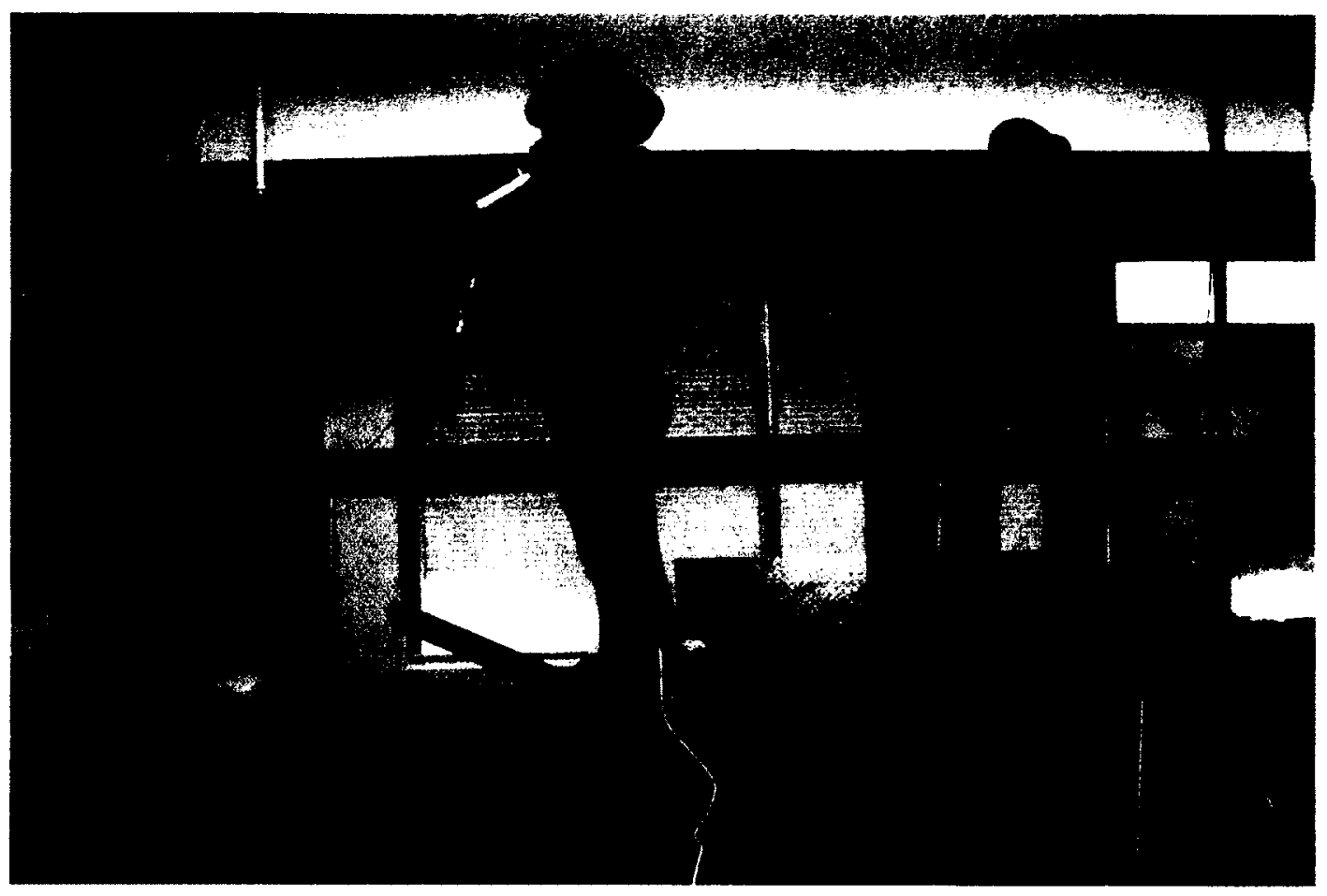

Figure 4.1: Data Acquisition Procedure.

procedure for all the volunteers who participated for research project. Prior to starting the data collection process, subjects were asked to step with their right foot on the load plate mounted at platform base. Coordination of kinematic data with load cell data was achieved by setting the origin of the GCS of Opti-track at the corner of the force plate. Once the subject was ready on the platform, the platform was raised to the neutral position by engaging it and the virtual skeleton of the subject was determined using the ARENA software. Then, the Tek Scan insoles were connected to the network hub and were calibrated using the Tek Scan step calibration procedure. Calibration of each foot required 10 seconds. In addition, insoles calibration was synchronized with the load cell calibration by asking subjects to stand on each foot at the centre of the force plate for 10 seconds. The BB1LP system settings were verified for respirometry data as well as metabolic energy expenditure data collection through the Logger Pro meter. Before collecting data, subjects were 
instructed to maintain upright stance with arms hanging at the sides and feet in line with the frontal plane. To begin the process, subjects were allowed to maintain a T-pose facing along the positive z-axis of the calibrated Opti-track GCS, which represented the anterior-posterior (A-P) axis of the body. The T-pose made actual data collection much easier for ARENA by enabling it to snap a pre-generated skeleton to the subject marker positions. After the T-pose, platform motion was started for actual data collection using the corresponding procedure. While the platform was in motion, participants were instructed to take first three deep breathes while in the T-pose position and tap their right foot on load plate with exhalation of third deep breath in order to start synchronized data collection using all equipment. 12 Motion profiles were run in random order for experimental trials. Each motion profile lasted for 360 seconds and there was a rest period of 60 seconds between successive runs of two motion profiles while data files were saved and processed. The subjects were instructed to maintain their balance at all times as they experienced each motion profile. Data was saved using the corresponding software for later analysis. The data collection procedure took 120 to 150 minutes per subject for all 12 motion profiles if all initial set-up and calibration was performed efficiently. In this way, experimental data of 14 participants was collected during laboratory experimentation.

\subsection{Data Sampling}

The data sampled by various modules of the system were synchronized. Since, the Opti-track system captures motion data at $100 \mathrm{~Hz}$, the BB1LP and foot pressure sensors also sampled at the same frequency. This resulted in a total of 36,000 frames of data for 6 minutes of motion for each motion profile. 


\subsection{Data Post-processing}

This section outlines the necessary post-processing steps that were used to obtain accurate data for reliable analysis.

\subsubsection{Opti-track Data Smoothing}

Marker occlusion during motion capture is a potential major source of data error. This was caused mainly by safety railings and their protective padding that were located around the platform for subject safety. These railings could block some of the markers on the subject's body, impairing the camera's ability to collect the motion capture data for a segment of the body at few frames at a time. In addition to this, the markers could be swapped with each other due to contortion of the skeletal model during data playback. The Opti-track is equipped with built-in software for removal of such data gaps. The removal of marker swapping was performed manually using the ARENA data editing package through a relatively simple process. The main challenge was with filling of the data gaps. This was a very time-consuming process. The ARENA is equipped with 3-types of interpolations for gap filling: linear, cubic spline, and rigid body angle interpolation. For gaps less than 5 frames, i.e., 0.05 seconds, Opti-track recommends cubic spline interpolation. For longer gaps, i.e., 5 frames or greater, the rigid body angle interpolation is recommended. Although angle interpolation is considered to be less accurate than the cubic spline. Once gaps were filled, Opti-track data was smoothed using a data smoothing tool which is also available in the ARENA data editing package. This tool allows application of $6 \mathrm{~Hz}$ low pass filtering for smoothing of each body segment data by removing high frequency noise components. 


\subsubsection{Load Cell Data Filtering}

Load cell data have a noise factor, impairing the data quality for further processing. This noise component needed to be removed using filtering techniques. For this, we used two post-processing steps: 10 point down-sampling and Butterworth filtering. The load cell data was collected at $1000 \mathrm{~Hz}$, which was down-sampled on a 10 point scale so as to provide less noisy data at $100 \mathrm{~Hz}$. Further, this ground reaction force and moment data was smoothed using two-pass, fourth-order Butterworth filter (if necessary) by removing noise components to render the data to be used for further analysis. 


\section{Chapter 5}

\section{Multibody Dynamic Model Development}

\subsection{Introduction}

This chapter describes dynamic modelling of the human body incorporating threedimensional geometry by discretizing the body into multiple rigid body segments. To this end, body segment parameters are first estimated using the Dempster et al (courtesy of Miller and Nelson) anthropometric data [29]. Second, the NewtonEuler dynamics formulation is used to write equations of motion to evaluate applied forces and moments for each successive rigid body segment of the human model. Further, this approach is extended to calculate mechanical work performed by each joint in maintaining postural stability. The model can be used to calculate the total mechanical work done by the whole human body by summing work performed by individual joints in the body. In general, the multibody dynamic model development follows an inverse-dynamics approach where forces and moments are determined for the kinematics of the system.

\subsection{Multibody Dynamic Model}

For multibody dynamic model development, the following steps are performed: 
1. Three-dimensional kinematic analysis;

2. Three-dimensional kinetic analysis; and

3. Calculation of mechanical work performed by all body joints.

\subsubsection{Three-dimensional Kinematic Analysis}

Basically, human motion capture is defined as capturing or recording of real-life movements of a human in three dimensions. Many laboratories, which conduct analysis of human movements, utilize multiple cameras, load cells, and force plates. A multicamera system is important for accurate reconstruction of marker data. In addition to this, a load cell and associated force plate provides a means of measuring external forces and moments acting on the human body. These are input into a multibody dynamic model as known values. The flowchart in Fig. 5.1 outlines how a multibody dynamic model is used to analyse the acquired kinematic data. Firstly, threedimensional marker position data is streamed from the ARENA software, which is smoothed to eliminate noise and other effects before further processing. Next, anthropometric data that defines joint centres; centre of mass (CoM); length and mass, and density of various segments is read from an anthropometric data table. Since there are two frames of reference for motion data (described subsequently), a rotational transformation is applied to transform data from the global frame of reference into the local reference frame. Using the position data, linear velocities and accelerations are obtained by differentiation. Angular kinematic techniques are applied to determine Euler angles (which define segment orientation between two reference frames) and joint angles (which define joint relative orientation). Euler angles can be differentiated to obtain angular velocities and angular accelerations. Segment orientation data (i.e., Euler angles) are smoothed to improve accuracy in the estimation of angular velocities and accelerations that are used to calculate moments using the 


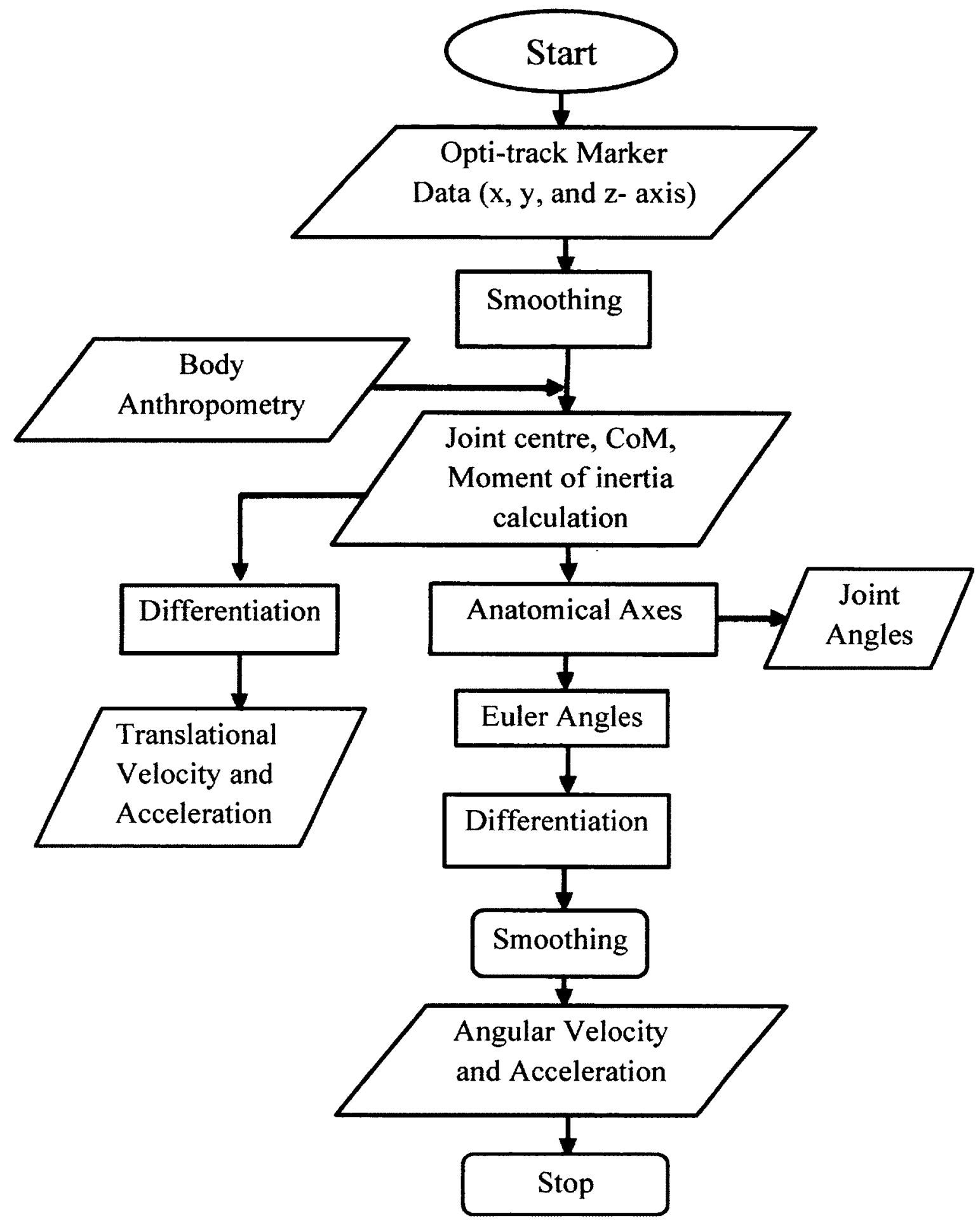

Figure 5.1: Flow chart for 3-D kinematic data analysis. 
equations of motion.

\section{Marker Data}

Marker data refers to observational data in the form of marker position as a function of time in three respective axes (i.e., $x, y$, and $z$ axes). Since the three-dimensional marker data define the position of different segments in three-dimensional space as a function of time, this as well as orientation data are used to evaluate the threedimensional kinematic parameters as outlined in the flowchart.

\section{Coordinate Systems}

Basically, two coordinate system are required for three-dimensional analysis of motion data. These are defined as the global (inertial) coordinate system (GCS) and the local (non-inertial) coordinate system (LCS).

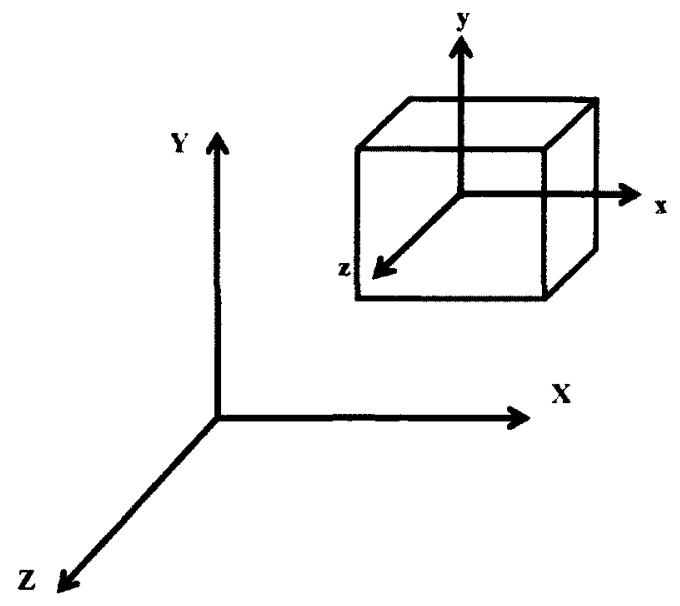

Figure 5.2: Global (XYZ) and Local (xyz) Coordinate Systems.

\section{i. Global Coordinate System}

The global coordinate system (GCS) or inertial reference system (IRS), which was identified as the laboratory coordinate system, is used to position an object 
in three dimensions as a function of time. In the context of the Opti-track system, this is regarded as orthogonal reference system which represents fixed axes for the laboratory. Generally, the global coordinate system (GCS) or the inertial frame axes are denoted in upper-case letters having an arbitrary origin defined at the centre of the motion platform, with the $\mathrm{X}$-axis representing the medio-lateral (M-L), the $\mathrm{Y}$-axis the axial (longitudinal), and $\mathrm{Z}$-axis the anteriorposterior (A-P) directions of the human body [56].

\section{ii. Local Coordinate System}

The local coordinate system (LCS) was defined as the reference frame that is fixed to each body segment. The origin of the local coordinate system is located at the centre of mass (CoM) of the rigid body segment. The local coordinate systems are denoted by lower-case letters with the $\mathrm{x}$-axis representing the mediolateral (M-L), the $y$-axis the vertical (longitudinal), and $z$-axis representing the anterio-posterior (A-P) directions of the body segment. By defining the position of the LCS with respect to the GCS, the positions of different body segments are established in three-dimensional space at any instant of time. It is noted that the definition of the LCS for each body segment is defined by the position of markers located on that segment [56].

\section{Transformation Matrix}

Transformation between reference systems is carried out to transform the position and orientation of the rigid body in one coordinate system to another coordinate system. Three markers were used on each body segment to completely define a non-coplanar rigid body in its local coordinate system in three-dimensional space. Therefore, the position and orientation of that segment with respect to the global frame can be completely determined by applying a transformation between the local 
and the global system [56]. In general, a rigid body is capable of undergoing both translation and rotation, where translation is defined as the change in position of its centre of mass (CoM), while rotation is defined as re-orientation of the body about its centre of mass (CoM).

\section{i. Translation or Linear Transformation}

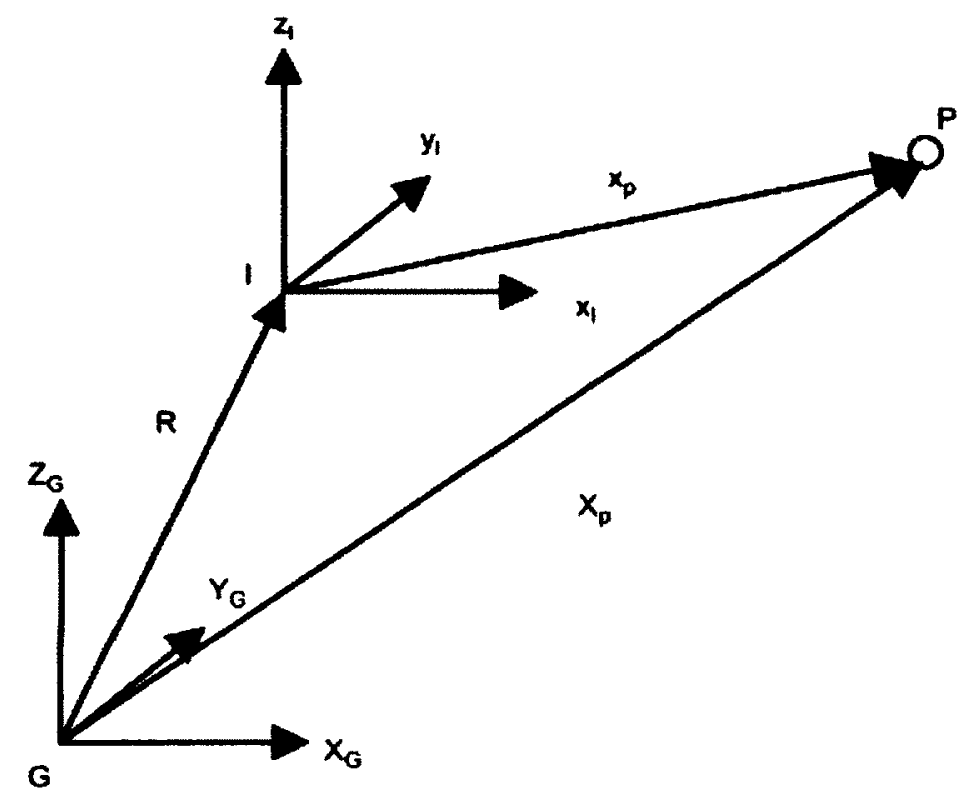

Figure 5.3: Linear transformation of local coordinate system $x_{l} y_{l} z_{l}$ to global coordinate system $X_{G} Y_{G} Z_{G}[61]$

For linear transformation between two coordinate systems, a vector $\vec{R}$ can be used. Let us consider $X_{G} Y_{G} Z_{G}$ as the global and $x_{l} y_{l} z_{l}$ as the local coordinate systems. In Fig. 5.3, $\vec{R}$ is a position vector that defines the relative position between the local and the global coordinate systems in XYZ with three components $\vec{R}_{X_{G}}, \vec{R}_{Y_{G}}$, and $\vec{R}_{Z_{G}}$ respectively. If there is no rotation in the LCS with respect to the GCS, the coordinates of a point $P$ identified as $P^{\prime}$ in the LCS can be transformed to its coordinates $P_{X_{G}}, P_{Y_{G}}, P_{Z_{G}}$ in the GCS as follows:

$$
\vec{P}=\vec{R}+\vec{P}^{\prime}
$$


or

$$
\left[\begin{array}{c}
P_{X_{G}} \\
P_{Y_{G}} \\
P_{Z_{G}}
\end{array}\right]=\left[\begin{array}{c}
R_{X_{G}} \\
R_{Y_{G}} \\
R_{Z_{G}}
\end{array}\right]+\left[\begin{array}{c}
P_{x_{l}}^{\prime} \\
P_{y_{l}}^{\prime} \\
P_{z_{l}}^{\prime}
\end{array}\right]
$$

Conversely, conversion of the coordinates of point $\mathrm{P}$ from the GCS to the LCS is given as:

$$
\overrightarrow{P^{\prime}}=\vec{P}-\vec{R}
$$

or

$$
\left[\begin{array}{c}
P_{x_{l}}^{\prime} \\
P_{y_{l}}^{\prime} \\
P_{z_{l}}^{\prime}
\end{array}\right]=\left[\begin{array}{c}
P_{X_{G}} \\
P_{Y_{G}} \\
P_{Z_{G}}
\end{array}\right]-\left[\begin{array}{c}
R_{X_{G}} \\
R_{Y_{G}} \\
R_{Z_{G}}
\end{array}\right]
$$

In this way, the linear translation between two relative coordinate systems can be represented [61].

\section{ii. Euler Angles (Orientation Transformation)}

Orientation of a body segment with respect to a reference frame is commonly defined using Euler angles $\phi, \theta$, and $\psi$. A common order of rotations is the Cardan rotation sequence (xyz rotation sequence), which is applied for performing rotation about three axes. It is important to take the rotation sequence into consideration as Euler angle rotation is not commutative in nature. So, following the xyz rotation sequence from the GCS to the LCS, the first rotation takes place about the z-axis which changes the orientation of both the $\mathrm{x}$ and

$y$ axes. Second, rotation about the $y$-axis changes the orientation of both the $x$ and $z$-axes. The final rotation is about the $x$-axis, which results in the final 
orientation of the coordinate system, which re-orients a segment with respect to the inertial frame of reference [56]. In Fig. 5.4, the first rotation takes place by angle $\phi$ about the $\mathrm{X}$-axis. This leads to a new orientation of both the $\mathrm{Y}$ and $\mathrm{Z}$ axes. The second rotation through angle $\theta$ is about the current $\mathrm{Y}$-axis. The final rotation occurs through angle $\psi$ about the current Z-axis resulting in the final orientation of the coordinate system.

Let X4Y4Z4 represent the global coordinate system and X1Y1Z1 represent the local coordinate system. Then, the orientation of the global coordinate system relative to the local coordinate system, designated by the matrix $R_{G}^{L}$ is the result of three successive rotations $z R_{G}^{A^{\prime \prime}}, y R_{A^{\prime \prime}}^{A^{\prime}}$, and $x R_{A^{\prime}}^{L}$ where $A^{\prime \prime}$ and $A^{\prime}$ represent intermediate coordinate systems that were obtained due to Euler angle rotations $\psi$ and $\theta$ respectively; i.e.,
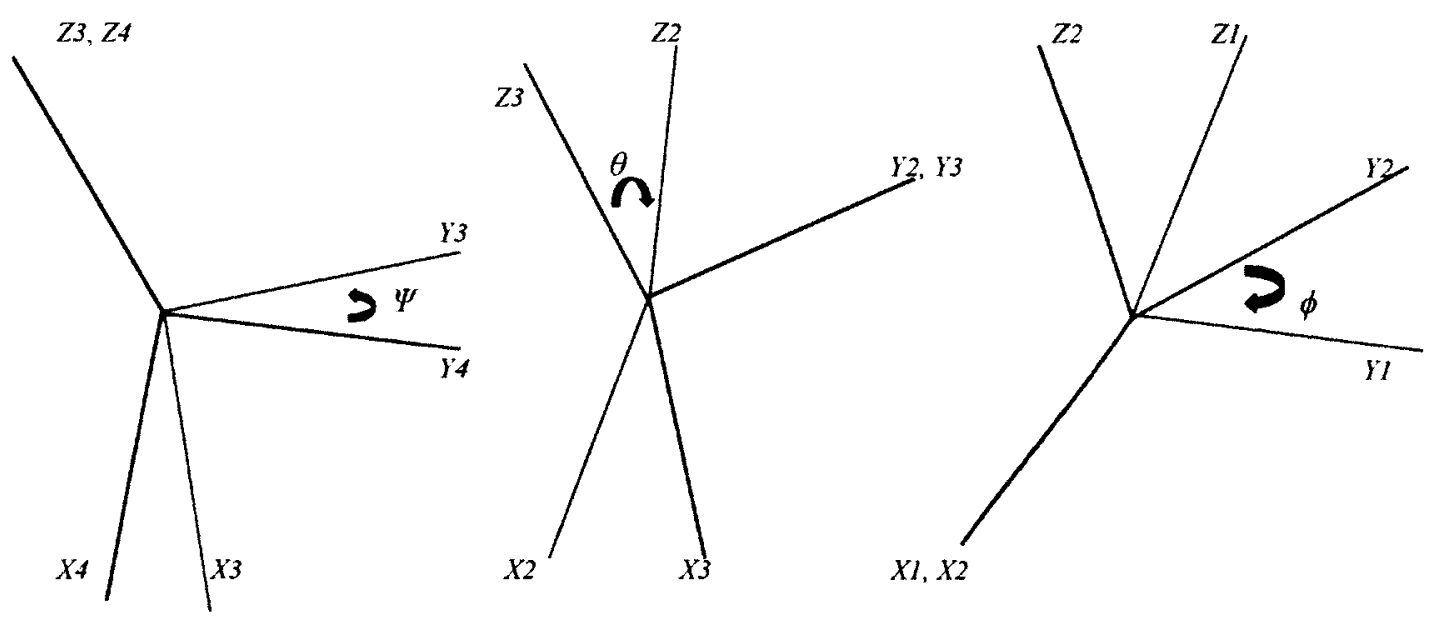

Figure 5.4: Euler angle rotation sequences, where $\mathrm{X} 4 \mathrm{Y} 4 \mathrm{Z} 4$ identifies the global coordinate system (GCS) and X1Y1Z1 the local coordinate system (LCS). 


$$
\left[R_{G}^{L}\right]=\left[x R_{A^{\prime}}^{L}\right]\left[y R_{A^{\prime \prime}}^{A^{\prime}}\right]\left[z R_{G}^{A^{\prime \prime}}\right]=\left[\begin{array}{ccc}
1 & 0 & 0 \\
0 & \cos \phi & -\sin \phi \\
0 & \sin \phi & \cos \phi
\end{array}\right]\left[\begin{array}{ccc}
\cos \theta & 0 & \sin \theta \\
0 & 1 & 0 \\
-\sin \theta & 0 & \cos \theta
\end{array}\right]\left[\begin{array}{ccc}
\cos \psi & -\sin \psi & 0 \\
\sin \psi & \cos \psi & 0 \\
0 & 0 & 1
\end{array}\right.
$$

Conversely, the transformation from the GCS to the LCS can be represented by identifying this transformation matrix as:

$$
R_{L}^{G}=\left[R_{G}^{L}\right]^{T}
$$

it can be shown that:

$$
\left[R_{L}^{G}\right]=\left[z R_{A^{\prime \prime}}^{G}\right]\left[y R_{A^{\prime}}^{A^{\prime \prime}}\right]\left[x R_{L}^{A^{\prime}}\right]
$$

so that:

$$
\left[\begin{array}{c}
X 4 \\
Y 4 \\
Z 4
\end{array}\right]=\left[z R_{A^{\prime \prime}}^{G}\right]\left[y R_{A^{\prime}}^{A^{\prime \prime}}\right]\left[x R_{L}^{A^{\prime}}\right]\left[\begin{array}{c}
X 1 \\
Y 1 \\
Z 1
\end{array}\right]
$$

with, 


$$
\left[R_{L}^{G}\right]=\left[\begin{array}{ccc}
\cos \theta \cos \psi & \sin \phi \sin \theta \cos \psi+\cos \phi \sin \psi & -\cos \psi \sin \theta \cos \phi+\sin \phi \sin \psi \\
-\sin \psi \cos \theta & -\sin \phi \sin \theta \sin \psi+\cos \phi \cos \psi & \cos \phi \sin \theta \sin \psi+\sin \phi \cos \psi \\
\sin \theta & -\cos \theta \sin \phi & \cos \phi \cos \theta
\end{array}\right]
$$

The combined matrix in Eq.(13) is known as decomposition matrix whose elements represent the orientation of the local coordinate system with respect to the global coordinate system. Cardan angles can be derived directly from the decomposition matrix [56]. The angle of the second rotation (axial rotation) can be evaluated from element $R_{31}$ of the decomposition matrix; using the following relationships.

Rotation about the y-axis, (axial rotation):

$$
\theta=\arcsin \left(R_{L 31}^{G}\right)
$$

Rotation about the x-axis, (flexion-extension):

$$
\phi=\arcsin \left(\frac{-R_{32}}{\cos \theta}\right)
$$

Rotation about the z-axis (abduction-adduction):

$$
\psi=\arcsin \left(\frac{-R_{21}}{\cos \theta}\right)
$$

Therefore, the Cardan angle sequence, i.e., $\phi, \theta$, and $\psi$, represents the relative orientation between the laboratory axis system and the anatomical axis system. These are important parameters for calculation of angular velocity and angular acceleration of different body segments. 


\section{Joint Coordinate System}

Joint coordinate systems are required for tracking joint angles. The selected method is the one which is recommended by the Standardization and Terminology Committee (STC) of the International Society of Biomechanics (ISB) which is based on the joint coordinate system proposed by Chao (1980) and Grood and Suntay (1983) for different body joints. This method was developed in order to clearly identify the anatomical meaning of three rotations that can occur between any two body segments [58]. A total of 14 body joints are considered for specifying joint angles in the human body. The joint coordinate system (JCS) approach considers the two local coordinate systems assigned to each of the two body segments constituting a joint. For example, to evaluate the joint angles through the right hip joint, coordinate systems of the pelvis segment and the right thigh segment are considered [58].
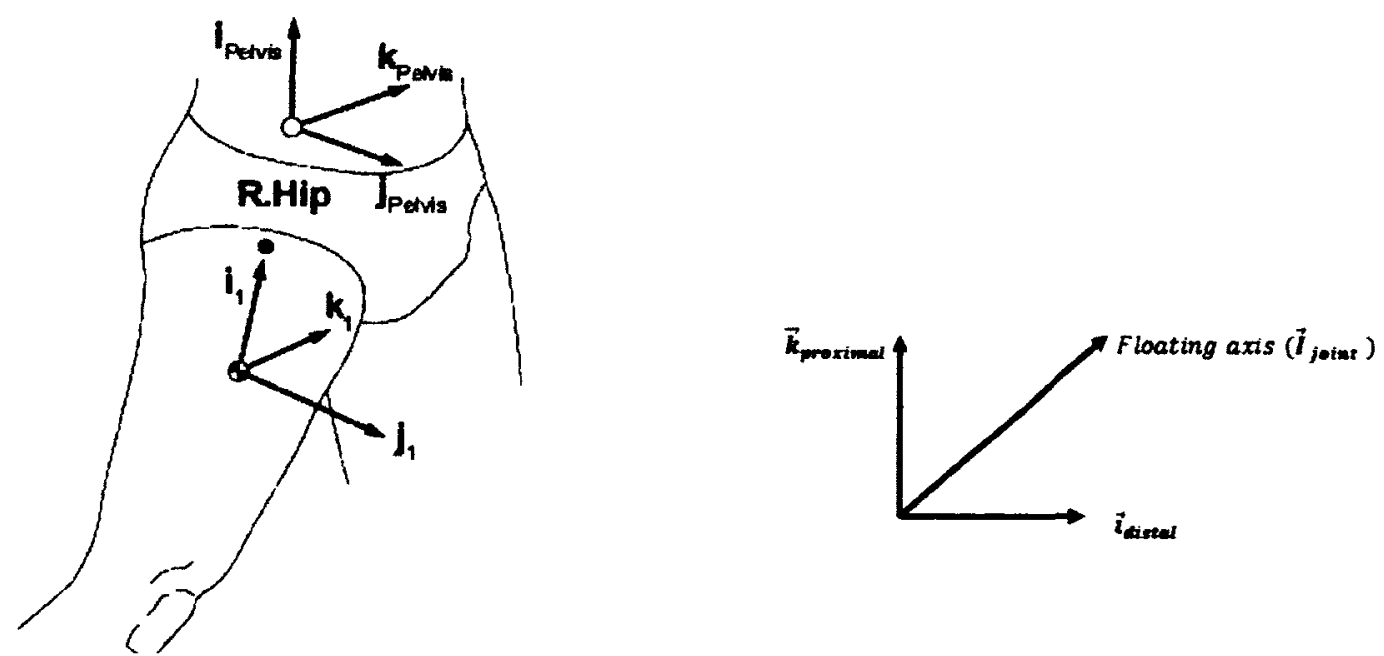

Figure 5.5: (a) Right hip joint angle calculations between pelvis and right thigh segments [59]; and (b) Floating axis [56].

In the right hip joint example (Fig. 5.5 (a)), the longitudinal axis $\vec{i}_{\text {distal }}=\vec{i}_{1}$ of the LCS of the distal segment (i.e., right thigh) and the medio-lateral axis $\vec{k}_{\text {proximal }}=$ $\vec{k}_{\text {pelvis }}$ of the proximal segment (i.e., the pelvis) are considered [59]. The cross product 
of the distal longitudinal axis with the proximal medio-lateral axis defines the floating axis (Fig. 5.5(b)), which is perpendicular to the plane formed by the directed axes as:

Floating Axis $\left(\vec{I}_{\text {joint }}\right)=\frac{\left(\vec{k}_{\text {proximal }} \times \vec{i}_{\text {distal }}\right)}{\left|\vec{k}_{\text {proximal }} \times \vec{i}_{\text {distal }}\right|}$

The angles of the joint are then denoted as:

$\alpha=$ flexion/extension angle;

$\beta=$ abduction/adduction angle; and

$\gamma=$ axial rotation angle where the flexion/extension angle identifies the rotation about the medio-lateral axis of the proximal segment, $\vec{k}_{\text {proximal }}$; the abduction/adduction is rotation about the floating axis, $\vec{I}_{\text {joint }}$; and the axial rotation is the rotation about the longitudinal axis of the distal segment $\vec{i}_{\text {distal }}[59]$. These three joint angles (measured in degrees) at the right hip joint are calculated as:

flexion/extension angle:

$\alpha=90-\arccos \left(\vec{i}_{\text {proximal }} \cdot \vec{I}_{\text {joint }}\right)$

abduction/adduction angle:

$\beta=-90-\arccos \left(\vec{k}_{\text {proximal }} \cdot \vec{i}_{\text {distal }}\right)$

axial rotation angle:

$\gamma=90-\arccos \left(\vec{k}_{\text {distal }} \cdot \vec{I}_{\text {joint }}\right)$

This technique is applied to represent the JCSs throughout the human body and to determine joint angles for all three axes. Each JCS is a non-orthogonal system because the vertical and lateral axes of two successive segments defining the floating axis are not necessarily perpendicular [59].

\subsubsection{Three-dimensional Kinetic Analysis}

Fig. 5.6 demonstrates the three-dimensional kinetic analysis approach which deals with evaluation of forces and moments that cause motion of the human body. Forces and moment calculations are further extended for estimation of mechanical work 


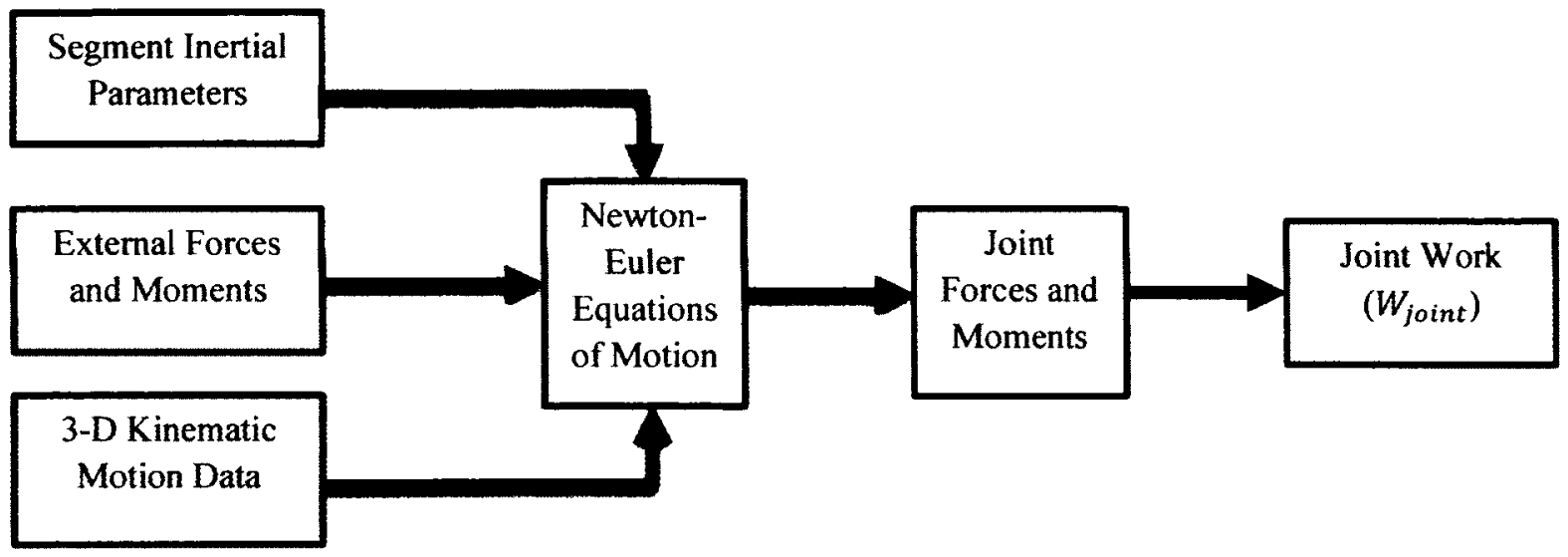

Figure 5.6: Inverse dynamics approach flow-chart for 3-D kinetic analysis [56].

performed by different body joints. Kinetic analysis is important because it allows researchers to investigate the basic mechanisms of movement. In biomechanics, two approaches are available for three-dimensional kinetic analysis. In the first approach, called forward dynamics, both forces and torques produced at different joints are used as input in order to predict human movement. In the second approach, called inverse dynamics, the joint forces and moments are determined using measured externallyapplied forces, segment kinematics, and segment inertial parameters as inputs. These two approaches are the reverse of one-another. In the present study, the inverse dynamics approach was used. The Newton-Euler method was used to derive the required equations of motion for all the body segments. The inverse dynamics technique shown in Fig. 5.6 was followed step by step for investigation of forces and moments.

\section{Anthropometry for Segment Characteristics}

Anthropometry is defined as a system to approximate the following characteristic data for various segments of the human body: mass, centre of mass, segment lengths, and principle moments of inertia. Anthropometric data are collected either from 


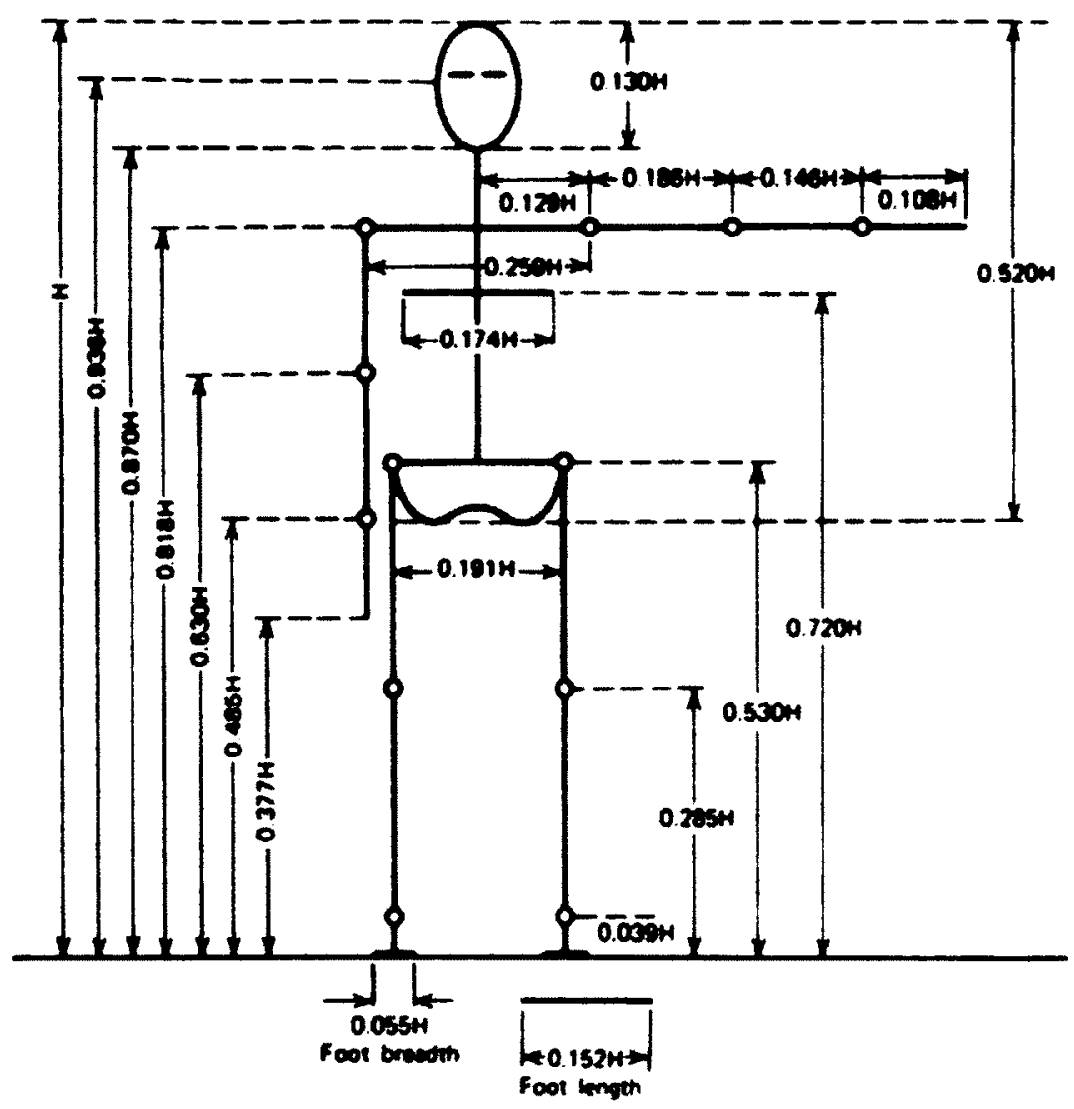

Figure 5.7: Body segment lengths approximated as a fraction of total body height (H) $[29]$.

cadaver studies (provided by Chadler, et al 1975; Dempster 1955, Clauser, et al 1969) or from direct measurements (provided by Brooks and Jacobs 1975) $[29,56]$. Clearly, no approach can be considered as perfectly accurate because anthropometric data characteristics vary from person to person. In this study, the data reported by Dempster 1955, was used for centres of mass, density, and mass of the segments $[29,82]$; whereas segment lengths were determined from the data reported by Drillis and Contini (1966) $[29,56]$, where each length is reported as a function of the total body height of the subject (Fig. 5.7). The mass moment of inertia of each segment was determined by idealizing the shape of the segment. The principle mass moments 
of inertia of a segment are represented as a diagonal matrix:

$$
[I]=\left[\begin{array}{ccc}
I_{x} & 0 & 0 \\
0 & I_{y} & 0 \\
0 & 0 & I_{z}
\end{array}\right]
$$

where diagonal elements $I_{x}, I_{y}$, and $I_{z}$ represent the mass moments of inertia of each segment with respect to its principal axes $\mathrm{x}, \mathrm{y}$, and $\mathrm{z}$.

Hanavan's Geometrical Model for Multibody Dynamic Model Development
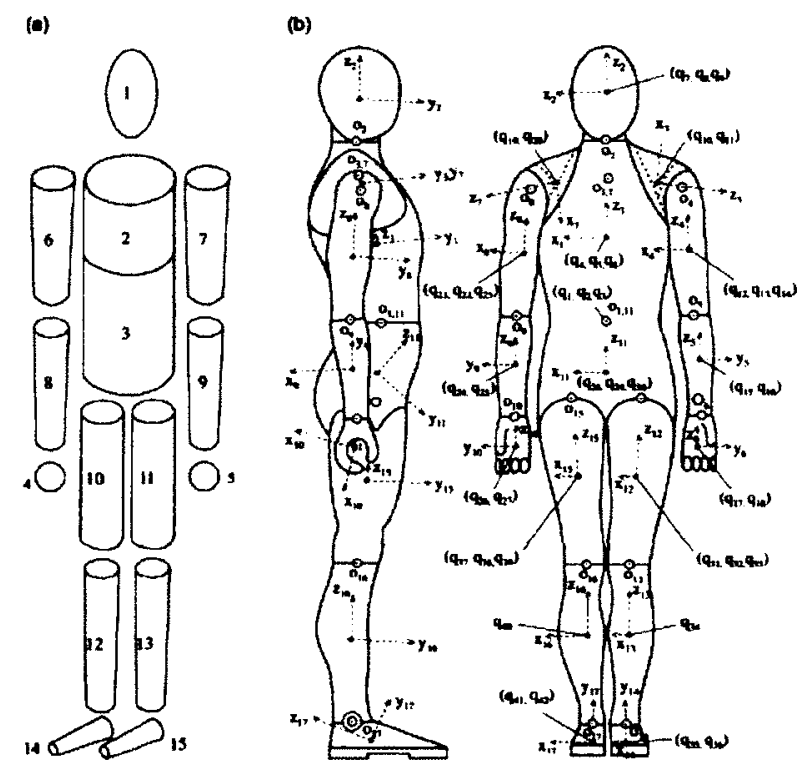

Figure 5.8: (a) Hanavan's 15 segmented geometrical human model; (b) Lateral and frontal view of Hatze's 17 segment humanoid [60].

Mathematical modelling of the human body is based on idealizing body segments as geometrical solid shapes, which may vary from simple shapes to more complex ones. A number of such models are available in the literature [60], such as Hanavan's 15 segment Model and Hatze's 17 segment humanoid model for mathematical 
modelling [56.60]. In this study, Hanavan's 15 body segment geometric model was adopted for human body modelling, because it represents the 15 segment rigid bodies with standard geometric shapes. In this model, the hands and head are idealized as ellipsoids (Fig. 5.9.(a)) and all other body segments: upper arms, lower arms, trunk, pelvis, thighs, shank, and feet are idealized as circular prisms (Fig. 5.9.(b)) The principal mass moments of inertia equations for these assumed solid geometric shapes are as follows:
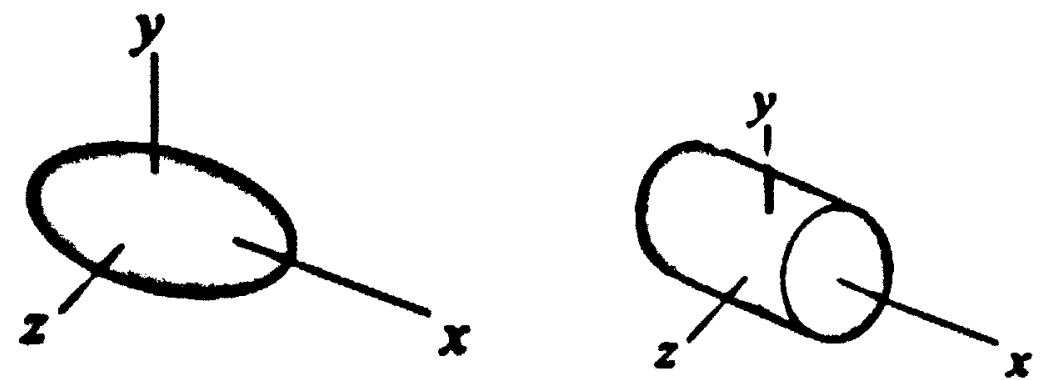

Figure 5.9: (a) Ellipsoid; and (b) Circular prism [56]

a For an ellipsoid (Fig. 5.9(a)), (i.e., for the head and hands):

$$
\begin{aligned}
& I_{x}=\frac{1}{5} m\left(y^{2}+z^{2}\right) \\
& I_{y}=\frac{1}{5} m\left(x^{2}+z^{2}\right) \\
& I_{z}=\frac{1}{5} m\left(x^{2}+y^{2}\right)
\end{aligned}
$$

where $m=$ mass, $x=$ depth, $y=$ height, and $z=$ width.

b For a circular prism (Fig. 5.9(b)) (i.e., for arms, legs, feet, trunk, and pelvis):

$$
I_{x}=\frac{1}{2} m r^{2}
$$




$$
\begin{aligned}
& I_{y}=\frac{1}{12} m\left(3 r^{2}+l^{2}\right) \\
& I_{z}=\frac{1}{12} m\left(3 r^{2}+l^{2}\right)
\end{aligned}
$$

where $m=$ mass of the prism, $l=$ length of the prism, and $r=$ radius.

Using the moment Equations (Eqns. 18 through 23) for representation of the principal mass moments of inertia, values for the 15 body segments were generated using segment lengths and mass anthropometric data $[29,56]$.

\section{Segment Centre of Mass (CoM)}

Segment centre of mass is defined as the point in the segment where the entire mass of the segment can be assumed to be concentrated for the purpose of determining translational dynamies. The longitudinal axis (y-axis) of a segment is defined as the connecting line between the proximal and distal virtual joint centres constituting a segment. It is assumed that the centre of mass lies between the proximal and distal joint centres along the longitudinal axis. For instance, if $\overrightarrow{C_{1}}$ and $\vec{C}_{2}$ locate the virtual joint centres of a segment and $d_{1}$ is the distance from the proximal virtual joint centre to the centre of mass, which is assumed to be in the middle of the segment [56]; then, the centre of mass (CoM) can be located as [56]:

$$
\vec{O}_{C o M}=\vec{C}_{1}+d_{1}\left(\vec{C}_{2}-\vec{C}_{1}\right)
$$

where distance $d_{1}$ can be obtained from the anthropometric data (Dempster, 1955) [56]. The centre of mass is evaluated in the local frame of reference (or anatomical frame of reference). 


\section{Three-dimensional Kinematic Data}

The three-dimensional kinematic data determines the linear and angular positions, velocities, and accelerations, which are used with inverse dynamics to estimate forces and moments of different body segments. The marker data provide the position and orientation of each body segment in the LCS with respect to the GCS. Specifically, Euler angles, i.e., $\phi, \theta$, and $\psi$, define the orientation between the two reference frames, which depict the angular orientation of the body segment in three-dimensional space. As the segment moves, the angular orientation is differentiated as an intermediate step toward obtaining the angular velocity components of the segment,

$$
\begin{gathered}
\dot{\phi}_{i}=\frac{d \phi}{d t} \approx \frac{\phi_{i+1}-\phi_{i-1}}{2 \Delta t} \mathrm{rad} / \mathrm{sec} \\
\dot{\theta}_{i}=\frac{d \theta}{d t} \approx \frac{\theta_{i+1}-\theta_{i-1}}{2 \Delta t} \mathrm{rad} / \mathrm{sec} \\
\dot{\psi}_{i}=\frac{d \psi}{d t} \approx \frac{\psi_{i+1}-\psi_{i-1}}{2 \Delta t} \mathrm{rad} / \mathrm{sec}
\end{gathered}
$$

where $2 \Delta t$ is the time interval between the $(i-1)^{t h}$ and the $(i+1)^{s t}$ angle.

Using these components, angular velocities $\omega_{x}, \omega_{y}$, and $\omega_{z}$ of each segment are determined as:

$$
\omega_{i}=\left[\begin{array}{c}
\omega_{x i} \\
\omega_{y i} \\
\omega_{z i}
\end{array}\right]=\left[\begin{array}{ccc}
\cos \theta \cos \psi & \sin \psi & 0 \\
-\cos \theta \sin \psi & \cos \psi & 0 \\
\sin \theta & 0 & 1
\end{array}\right]\left[\begin{array}{c}
\dot{\phi}_{i} \\
\dot{\theta}_{i} \\
\dot{\psi}_{i}
\end{array}\right]
$$

Next, angular accelerations can be evaluated by differentiation of the angular velocities: 


$$
\begin{aligned}
& \alpha_{x i}=\frac{d \omega_{x}}{d t}=\frac{\omega_{x i+1}-\omega_{x i-1}}{2 \Delta t} \mathrm{rad} / \mathrm{sec}^{2} \\
& \alpha_{y i}=\frac{d \omega_{y}}{d t}=\frac{\omega_{y i+1}-\omega_{y i-1}}{2 \Delta t} \mathrm{rad} / \mathrm{sec}^{2} \\
& \alpha_{z i}=\frac{d \omega_{z}}{d t}=\frac{\omega_{z i+1}-\omega_{z i-1}}{2 \Delta t} \mathrm{rad} / \mathrm{sec}^{2}
\end{aligned}
$$

Finally, the translational acceleration of the CoM of each segment is determined as:

$$
A_{i}=\frac{d_{i+1}-2 d_{i}+d_{i-1}}{\Delta t^{2}} \mathrm{~m} / \mathrm{sec}^{2}
$$

where $\mathrm{d}$ is the displacement of the CoM for translational acceleration calculations [29].

\section{External Forces}

The third type of data that was acquired during the experimental work was the external forces and moments exerted on the subject by the outside environment. The most significant external force is the ground reaction force (GRF) and moment. In general, the ground force has a dominating vertical force component $\left(F_{y}\right)$, acting along the weight of the body with two shear force components $F_{x}$ and $F_{z}$, acting along the medio-lateral and anterio-posterior directions, respectively. In addition, three moment components $\left(M_{x}, M_{y}\right.$, and $\left.M_{z}\right)$ in the respective medio-lateral, vertical, and anterio-posterior directions can also exist. The load cell with the force plate along with the foot pressure sensor system were used to acquire these six force and moment components, i.e., $F_{x}, F_{y}, F_{z}, M_{x}, M_{y}$, and $M_{z}$ applied to the right foot of the subject. These values are incorporated into the equations of motions of the right foot segment in the multibody dynamic model. It was necessary to synchronize ground reaction force data with the kinematic data. This was achieved by establishing the origin of 
the GCS at the force platform origin. Moreover, using the inverse dynamic approach, ground reaction force and moment components acting on the left foot were calculated and validated against the recordings of the Tek Scan pressure sensor system for the left foot.

\section{Three-dimensional Inverse Dynamics Approach}

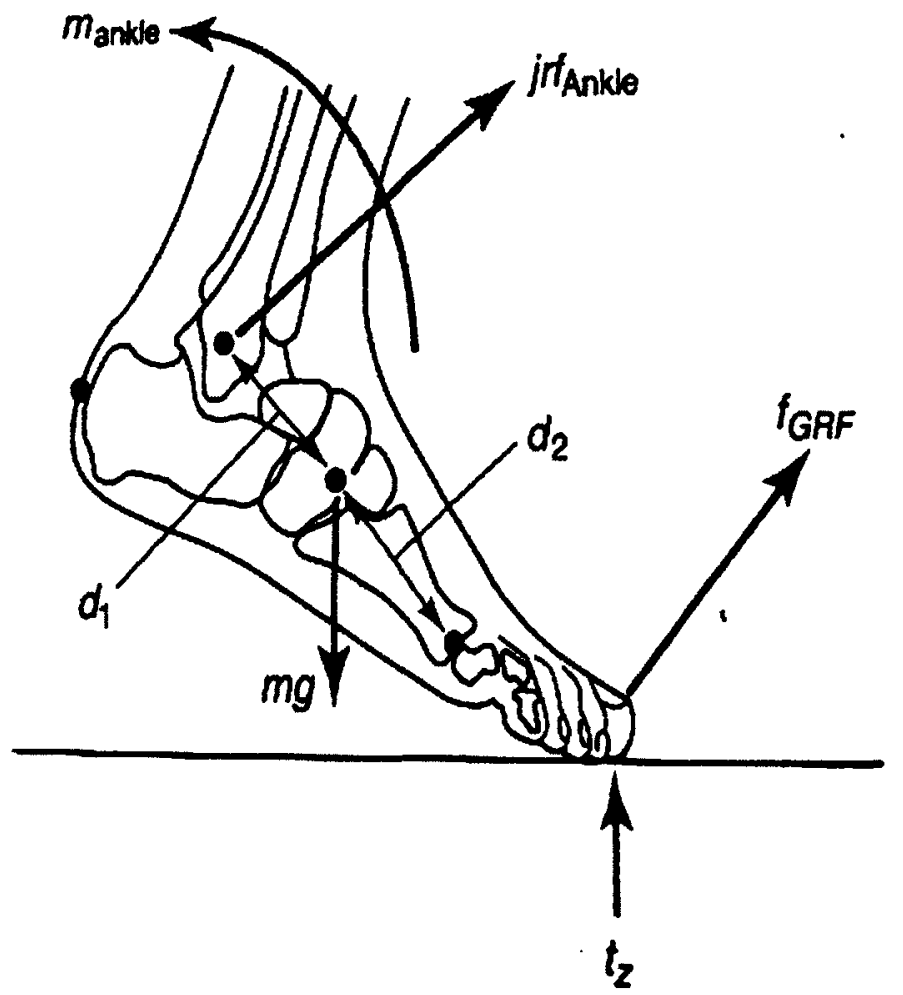

Figure 5.10: 3-D inverse dynamics approach for foot segment [56].

A foot segment is presented below as an example to develop the corresponding force and moment equations for three-dimensional kinetic analysis (Fig. 5.10). The translational dynamic equation for the ankle joint reaction force in vector form can be represented as:

$$
\vec{F}_{a n k l e}=j r f_{A n k l e}=m \vec{a}_{C o M}-m \vec{g}-\vec{f}_{G R F}
$$


where, $j r f_{A n k l e}$ is the ankle joint reaction force and $\vec{a}_{C o M}$ is the translational acceleration of the centre of mass of the segment, $m \vec{g}$ is the segment weight vector, and $\vec{f}_{G R F}$ is the ground reaction force. This translational dynamic equation of $\vec{F}_{\text {Ankle }}$ can be expressed in terms of components $\left(F_{x}, F_{y}\right.$, and $\left.F_{z}\right)$ in $\mathrm{x}, \mathrm{y}$, and $\mathrm{z}$ directions representing the medio-lateral, longitudinal, and anterior-posterior directions respectively:

$$
\begin{aligned}
& F_{x}=j r f_{\text {Ankle }_{x}}=m a_{C o M_{x}}-m g_{x}-f_{G R F_{x}} \\
& F_{y}=j r f_{\text {Ankle }_{y}}=m a_{C o M_{y}}-m g_{y}-f_{G R F_{y}} \\
& F_{z}=j r f_{\text {Ankle }_{z}}=m a_{C o M_{z}}-m g_{z}-f_{G R F_{z}}
\end{aligned}
$$

where, $j r f_{\text {Ankle }_{x}}, j r f_{\text {Ankle }_{y}}$, and $j r f_{\text {Ankle }_{z}}$ are force components in the medio-lateral, longitudinal, and anterior-posterior directions respectively; $a_{C o M_{x}}, a_{C o M_{y}}$, and $a_{C o M_{z}}$ are translational acceleration components in the three respective directions; and $f_{G R F_{x}}, f_{G R F_{y}}$, and $f_{G R F_{z}}$ are the three ground reaction force components; and $m g_{x}$, $m g_{y}$, and $m g_{z}$ define the weight vector in three dimensional space [56].

The rotational dynamic equation about the CoM is:

$$
\vec{M}_{\text {Ankle }}=j \vec{M}_{\text {ankle }}=[I] \vec{\alpha}+\vec{\omega} \times[I] \vec{\omega}-\left(\vec{d}_{1} \times j r \vec{f}_{\text {Ankle }}\right)-\left(\vec{d}_{2} \times \vec{f}_{G R F}\right)-\vec{t}
$$

where, $j \vec{M}_{\text {Ankle }}$ is the ankle moment vector; I is the mass moment of inertia matrix for the foot segment; $\vec{\alpha}$ is the angular acceleration; $\vec{d}_{1} \times j r \vec{f}_{\text {Ankle }}$ is the moment of $j r f_{\text {Ankle }}$ about the CoM, which is the cross-product of the proximal vector from the centre of mass (CoM) of the foot segment to the proximal end of the foot segment with the joint reaction force. Further, $\vec{d}_{2} \times \vec{f}_{G R F}$ is another moment vector component, which is the cross-product of the distal vector of the foot segment from the centre of mass 
(CoM) to the distal end of the foot segment with the ground reaction force (GRF) vector, and $\vec{t}$ is the ground reaction moment vector [56]. In terms of components, Eq. (36) can be expressed as:

$$
\begin{aligned}
& M_{x}=M_{\text {ankle }_{x}}=I_{x x} \alpha_{x}+\left(I_{z z}-I_{y y}\right) \omega_{z} \omega_{y}-M_{j r f_{x}}-M_{G R F_{x}}-t_{x} \\
& M_{y}=M_{\text {ankle }}=I_{y y} \alpha_{y}+\left(I_{x x}-I_{z z}\right) \omega_{x} \omega_{z}-M_{j r f_{y}}-M_{G R F_{y}}-t_{y} \\
& M_{z}=M_{\text {ankle }}=I_{z z} \alpha_{z}+\left(I_{y y}-I_{x x}\right) \omega_{y} \omega_{x}-M_{j r f_{z}}-M_{G R F_{z}}-t_{z}
\end{aligned}
$$

where, $\vec{M}_{j r f}=\left(\vec{d}_{1} \times j r \vec{f}_{\text {ankle }}\right)$ and $\vec{M}_{G R F}=\left(\vec{d}_{2} \times \vec{f}_{G R F}\right)$

In Eqns. 37, 38, and 39, $I_{x x}, I_{y y}$, and $I_{z z}$ represent components of the moment of inertia vectors and $\alpha_{x}, \alpha_{y}$, and $\alpha_{z}$ are components of segment angular accelerations. $I \vec{\alpha}$ should be expressed relative to the inertial frame of reference. Because angular velocities are expressed in the principal axis coordinate system of the rigid body segment, which is rotating with respect to the GCS (i.e., inertial frame of reference), the moment of inertia in the GCS can be represented as:

$$
\left(\frac{d L}{d t}\right)_{G C S}=\left(\frac{d L}{d t}\right)_{L C S}+(\vec{\omega} \times \vec{L})
$$

So, $\vec{\omega} \times \vec{L}$ is considered to be included in the rotational dynamic equations during three-dimensional kinetic analysis.

In this way, for the three-dimensional inverse dynamic modelling approach, calculations proceed from the distal to the proximal joint of each segment. Proximal calculations of one segment are used for distal evaluations of the adjacent segment. This approach generates the six equations of motion for all 15 body segments.

However, all force and moment calculations which are supposed to be calculated 
in the local coordinate system (LCS) of each body segment, can be transformed into the GCS by applying the necessary transformation matrices, i.e.,

$$
\begin{aligned}
{\left[j R \vec{F}_{\text {ankle }}\right] } & =\left[T_{A T 2 G}\right]\left[j r \vec{f}_{\text {Ankle }}\right] \\
{\left[j \vec{M}_{\text {ankle }}\right] } & =\left[T_{A T 2 G}\right]\left[j \vec{m}_{\text {ankle }}\right]
\end{aligned}
$$

where, $\left[j r \vec{f}_{A n k l e}\right]$ and $\left[j \vec{m}_{a n k l e}\right]$ are the force and moment vectors in the LCS; $\left[j R \vec{F}_{a n k l e}\right]$ and $\left[j \vec{M}_{\text {ankle }}\right]$ are the force and moment vectors in the GCS; and $\left[T_{A T 2 G}\right]$ is the transformation matrix from the anatomical frame to the global reference frame [56].

\section{Newton-Euler Equations of Motion Estimation}

To develop the multibody dynamic model, Newton-Euler equations of motion were derived in a general way for all 15 body segments to determine the forces and moments exerted at the joints. Three different approaches were implemented for determining the results from the multibody dynamics model.

\section{i. Linked Chain Model}

The Linked Chain-Segmental Model has been regarded as the fundamental approach for human gait analysis and whole body force and torque estimation while walking or performing various other activities. In this approach, the human body is modelled as an open chain system of individual rigid body links interconnected at the joints. Newtonian mechanics was applied at each individual rigid body segment for calculation of inter-segmental joint reaction forces and moments, while starting at one end of the chain and proceeding to the other [86]. In this way, six equations of motion were derived for mathematical 

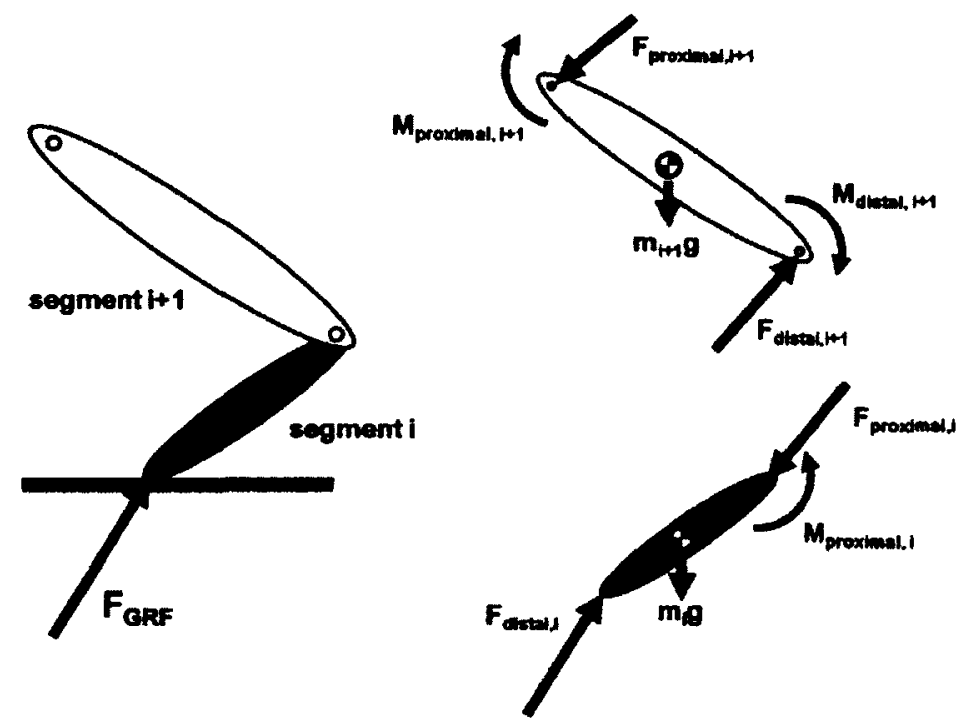

Figure 5.11: Linked chain model of body segments [86].

modelling of each body segment, which were based on the following assumptions:

- All joints are frictionless;

- Segments are considered as rigid bodies having distributed mass; and

- No agonistic-antagonistic muscle contraction is assumed (i.e., net muscle action is considered).

On the basis of this, joint reaction force and moment equations are arranged such as:

$$
\begin{gathered}
\vec{F}_{\text {proximal }_{i}}=M \vec{a}-\vec{F}_{\text {Distal }_{i}} \\
\vec{M}_{\text {proximal }_{i}}=[I] \vec{\alpha}+\vec{\omega} \times[I] \vec{\omega}-\vec{M}_{\text {Distal }_{i}}
\end{gathered}
$$

The arrangement of force and moment equations between two adjacent segments is based on the fact that force exerted by the distal segment on the proximal segment is equal and opposite to the force exerted by the proximal on the distal 
segment [86]. For instance, as shown in Fig. 5.11:

$$
\vec{F}_{\text {proximal }_{i}}=-\vec{F}_{\text {distal }_{i+1}}
$$

where $\vec{F}_{\text {proximal }}$ is the force exerted by the proximal segment on the distal segment and $-\vec{F}_{\text {distal }_{i+1}}$ is force exerted by the distal segment on the proximal segment [86].

Basically, two linked chains were formulated to derive equations of motion for the whole body . Top-down and bottom-up strategies were applied for upper and lower extremities separately. In the top-down approach for the upper body, analysis started from the hand extremities and ended at the trunk segment through the lower arms, upper arms, and including head segments simultaneously with the upper arms; and 6 equations of motion for each consecutive segment were solved. Similarly for the lower body, a similar chain started from the right foot following the bottom-up strategy with the GRF components as known external loading (obtained from the load cell and force plate data). The system is solved for forces and moments at each joint terminating at the left foot through the shanks and thighs. After solving both chains separately, all 90 unknowns required for mathematical modelling of the human body were evaluated.

\section{ii. Half Segmented Half Matrix Approach}

In this approach, a combination of the chain-link solution and matrix formulation was carried out. The upper extremities were solved as individual links of a chain until the trunk was reached (in similar manner as in the linked chain model). Upon reaching the L5-S1 joint (trunk-pelvis) the number of unknowns 
become problematic due to the right thigh and left thigh forces acting simultaneously on the pelvis. Therefore, at that point a $42 \times 42$ matrix approach is applied for derivation of the equations of motions for the 7 lower extremity segments. So, for the lower extremities, all force and moment equations are arranged in a matrix as follows:

$$
[A]_{42 \times 42}[X]_{42 \times 1}=[B]_{42 \times 1}
$$

and it can be solved for unknown forces as:

$$
[X]_{42 \times 1}=[A]_{42 \times 42}^{-1}[B]_{42 \times 1}
$$

where $[X]_{42 \times 1}$ contains all the unknowns required for the equations of motion. Further, an inverse-matrix solution is applied to calculate all the forces and moments for the lower segments in the $[X]_{42 \times 1}$ column vector.

Applying this approach, it's possible to solve all the 90 unknowns for the 15 body segments by introducing measured GRF component data for the right foot from the load cell and solving for the left foot GRF components through the lower $42 \times 42$ matrix.

\section{iii. Full Body Matrix Approach}

In this approach, the 15 segments of the human body are taken into consideration where each segment introduces 6 unknowns. Application of the NewtonEuler equations of motion is used to populate a $90 \times 90$ matrix starting with the upper segments and proceeding all the way to the lower foot segments, so that 3 ground reaction force components; $F_{g r f_{x}}, F_{g r f_{y}}$, and $F_{g r f_{z}}$ and 3 moment components; $M_{g r f_{x}}, M_{g r f_{y}}$, and $M_{g r f_{z}}$ of the right foot (obtained from load cell data) are inserted into the right foot equations; and the left foot ground reaction 
force and moment components are estimated through the matrix solution. In this way, a total of 90 equations of motion were arranged in the $90 \times 90$ matrix and associated vectors.

The LU decomposition method was applied for estimation of the 90 force and moment unknowns. It provided the inverse matrix solution by factorization of the matrix as a product of lower and upper triangular matrices.

With the $90 \times 90$ full body matrix approach the resulting set of equations is arranged as:

$$
[A]_{90 \times 90}[X]_{90 \times 1}=[B]_{90 \times 1}
$$

correspondingly, it can be solved as:

$$
[X]_{90 \times 1}=[A]_{90 \times 90}^{-1}[B]_{90 \times 1}
$$

where $[A]_{90 \times 90}$ is the $90 \times 90$ matrix, $[X]_{90 \times 1}$ is the column vector where all 90 unknown force and moment components for the 15 segments are located, and $[B]_{90 \times 1}$ is again a column vector representing mass, velocities, and accelerations of the force and moment components which are already evaluated.

Assembly of Force and Moment Equations for the Full Matrix Approach According to the Newton-Euler equations of motion, for any body segment: $\sum$ Forces $=0$ and $\sum$ Moments $=0$ or

$$
\begin{gathered}
\vec{F}_{\text {Proximal }}+\vec{F}_{\text {Distal }}=M \vec{a} \\
\vec{M}_{\text {Proximal }}+\vec{M}_{\text {Distal }}=[I] \vec{\alpha}+\vec{\omega} \times[I] \vec{\omega}
\end{gathered}
$$


Therefore, the general force and moment equations for any segment are defined as:

$$
\vec{F}_{\text {proximal }}=m \vec{a}_{C o M}-m \vec{g}-\vec{f}_{\text {distal }}
$$

This can be defined using the three respective axes, such that:

$$
\begin{aligned}
& F_{x}=m a_{C o M_{x}}-m g_{x}-f_{\text {distal }_{x}} \\
& F_{y}=m a_{C o M_{y}}-m g_{y}-f_{\text {distal }_{y}} \\
& F_{z}=m a_{C o M_{z}}-m g_{z}-f_{\text {distal }_{z}}
\end{aligned}
$$

where $F_{x}, F_{y}$, and $F_{z}$ are the proximal force components in the medio-lateral, longitudinal, and anterior-posterior directions respectively; $a_{C o M_{x}}, a_{C o M_{y}}$, and $a_{C o M_{z}}$ are the translational acceleration components in three respective directions; $f_{\text {distal }_{x}}, f_{\text {distal }_{y}}$, and $f_{\text {distal }_{z}}$ are the three distal force components and $m g_{x}$, $m g_{y}$, and $m g_{z}$ are the gravity force components in three axes. Similarly, rotational equations of motion can be arranged for torque calculations. The general equation of rotation for any segment:

$$
\vec{M}_{\text {proximal }}=[I] \vec{\alpha}+\vec{\omega} \times[I] \vec{\omega}-\left(\vec{d}_{1} \times \vec{F}_{\text {Proximal }}\right)-\left(\vec{d}_{2} \times \vec{F}_{\text {Distal }}\right)-\vec{t}_{\text {Distal }}
$$

where $\vec{M}_{\text {proximal }}$ is the proximal moment vector, $I$ is the moment of inertia matrix for the particular segment, $\vec{\alpha}$ is the angular acceleration of the segment, $\left(\vec{d}_{1} \times \vec{F}_{\text {Proximal }}\right)$ is the moment vector component resulting from the crossproduct of the proximal distance from the centre of mass (CoM) of the body segment to reaction force vector on the proximal side with the joint reaction force acting at the proximal joint, $\left(\vec{d}_{2} \times \vec{F}_{\text {Distal }}\right)$ is the moment vector resulting 
from the cross product of the distal distance of the next joining segment from the centre of mass (CoM) to the distal end of that segment with the distal force vector $\vec{F}_{\text {Distal }}$ of the next segment, and finally $\vec{t}_{D i s t a l}$ is the distal torque vector. Further, these equations can also be arranged in three respective axes, such that:

$$
\begin{aligned}
& M_{x}=I_{x x} \alpha_{x}+\left(I_{z z}-I_{y y}\right) \omega_{z} \omega_{y}-M_{\text {Proximal }_{x}}-M_{\text {Distal }_{x}}-t_{\text {Distal }_{x}} \\
& M_{y}=I_{y y} \alpha_{y}+\left(I_{x x}-I_{z z}\right) \omega_{x} \omega_{z}-M_{\text {Proximal }_{y}}-M_{\text {Distal }_{y}}-t_{\text {Distal }_{y}} \\
& M_{z}=I_{z z} \alpha_{z}+\left(I_{y y}-I_{x x}\right) \omega_{y} \omega_{x}-M_{\text {Proximal }_{z}}-M_{\text {Distal }_{z}}-t_{\text {Distal }_{z}}
\end{aligned}
$$

Arrangement of the equations results in all $M \vec{a}$ and $I \vec{\alpha}$ components being defined in the $[B]_{90 \times 1}$ right hand side vector, and all unknown forces being located in the $[X]_{90 \times 1}$ column vector, so that the first three rows of the matrix represent forces, (i.e., $F_{x}, F_{y}$, and $F_{z}$ ) and the successive three rows represent moments/torques $t_{x}, t_{y}$, and $t_{z}$ respectively. In this way, there are 4 arrangements of $3 \times 3$ matrices for each segment in the $[A]_{90 \times 90}$ matrix, in which each segment possesses 6 rows and 6 columns (Eq. 60).

For any segment, arrangement of the six equations of motion takes place as 
follows:

$$
\left[\begin{array}{cccccc}
1 & 0 & 0 & \phi & \phi & \phi \\
0 & 1 & 0 & \phi & \phi & \phi \\
0 & 0 & 1 & \phi & \phi & \phi \\
0 & -d_{3} & d_{2} & 1 & 0 & 0 \\
d_{3} & 0 & -d_{1} & 0 & 1 & 0 \\
-d_{2} & d_{1} & 0 & 0 & 0 & 1
\end{array}\right]_{6 \times 6}\left[\begin{array}{c}
F_{x} \\
F_{z} \\
M_{x} \\
M_{y} \\
M a_{y} \\
M a_{z} \\
M_{x x} \alpha_{x}+\left(I_{z z}-I_{y y}\right) \omega_{z} \omega_{y} \\
I_{y y} \alpha_{y}+\left(I_{x x}-I_{z z}\right) \omega_{x} \omega_{z} \\
I_{z z} \alpha_{z}+\left(I_{y y}-I_{x x}\right) \omega_{y} \omega_{x}
\end{array}\right]_{6 \times 1}
$$

where all the known components of forces and moments, (i.e., $M \vec{a}$ and $I \vec{\alpha}$ ) are defined on the right hand side of the matrix. On applying the inverse matrix solution, Eq. (60) can be re-arranged as:

$$
\left[\begin{array}{c}
F_{x} \\
F_{y} \\
F_{z} \\
M_{y} \\
M_{z}
\end{array}\right]_{6 \times 1}=\left[\begin{array}{cccccc}
1 & 0 & 0 & \phi & \phi & \phi \\
0 & 1 & 0 & \phi & \phi & \phi \\
0 & 0 & 1 & \phi & \phi & \phi \\
0 & -d_{3} & d_{2} & 1 & 0 & 0 \\
d_{3} & 0 & -d_{1} & 0 & 1 & 0 \\
-d_{2} & d_{1} & 0 & 0 & 0 & 1
\end{array}\right]_{6 \times 6}^{-1}\left[\begin{array}{c}
M a_{x} \\
M a_{y} \\
M a_{z} \\
I_{x x} \alpha_{x}+\left(I_{z z}-I_{y y}\right) \omega_{z} \omega_{y} \\
I_{y y} \alpha_{y}+\left(I_{x x}-I_{z z}\right) \omega_{x} \omega_{z} \\
I_{z z} \alpha_{z}+\left(I_{y y}-I_{x x}\right) \omega_{y} \omega_{x}
\end{array}\right]_{6 \times 1}
$$

On the basis of the above arrangement for all 15 segments, a $90 \times 90$ matrix 
can be arranged as follows:

$$
\left[\begin{array}{c}
F_{x} \\
F_{y} \\
F_{z} \\
M_{x} \\
M_{y} \\
M_{z} \\
\cdots \\
M_{z}
\end{array}\right]_{90 \times 1}=\left[\begin{array}{cccccccc}
1 & 0 & 0 & \phi & \phi & \phi & \ldots & \ldots \\
0 & 1 & 0 & \phi & \phi & \phi & \ldots & \ldots \\
0 & 0 & 1 & \phi & \phi & \phi & \ldots & \ldots \\
0 & -d_{3} & d_{2} & 1 & 0 & 0 & \ldots & \ldots \\
d_{3} & 0 & -d_{1} & 0 & 1 & 0 & \ldots & \ldots \\
-d_{2} & d_{1} & 0 & 0 & 0 & 1 & \ldots & \ldots \\
\ldots & \ldots & \ldots & \ldots & \ldots & \ldots & 1 & \ldots \\
\ldots & \ldots & \ldots & \ldots & \ldots & \ldots & \ldots & 1
\end{array}\right]_{90 \times 90}^{-1}\left[\begin{array}{c}
B_{1} \\
B_{3} \\
B_{4} \\
B_{5} \\
B_{6} \\
\ldots \\
B_{n}
\end{array}\right]_{90 \times 1}
$$

where;

$$
\begin{aligned}
& B_{1}=M a_{x} ; B_{2}=M a_{y} ; B_{3}=M a_{z} ; B_{4}=I_{x x} \alpha_{x}+\left(I_{z z}-I_{y y}\right) \omega_{z} \omega_{y} \\
& B_{5}=I_{y y} \alpha_{y}+\left(I_{x x}-I_{z z}\right) \omega_{x} \omega_{z} ; B_{6}=I_{z z} \alpha_{z}+\left(I_{y y}-I_{x x}\right) \omega_{y} \omega_{x} \\
& B_{n}=I_{z z} \alpha_{z}+\left(I_{y y}-I_{x x}\right) \omega_{y} \omega_{x}
\end{aligned}
$$

After constituting this $90 \times 90$ matrix, a solution for all the unknown forces and moments required for the multibody dynamic model can be obtained.

\subsubsection{Multibody Dynamic Model Comparative Analysis}

Three different approaches have been outlined in the above section for the mathematical modelling of the human body to evaluate the mechanical work performed by different joints. Our first approach was the Linked Chain Segmental Model (LSM), which followed the two consecutive chains, i.e., top-down and bottom-up for the upper 
and lower extremities respectively to calculate intersegmental forces and moments. The upper chain terminated at the lumbo-sacral (L5-S1) joint, while the lower chain started from the right foot by considering the GRF components as known forces and moments (from the load plate) and terminated at the left foot, where the GRF components of this foot were calculated through the link strategy. The second approach was a combination of the Linked Chain-Segmental (LSM) and the $42 \times 42$ Half-Matrix approaches, through which all 90 inter-segmental forces and moments were estimated for modelling the human. For this, the upper chain was formulated in the same way as the Linked Approach so that it terminated at the L5-S1 joint, while for the lower body, a half-matrix was generated for the lower extremity chain and an inverse matrix solution was applied for force and moment calculation.

In the last approach, termed as the $90 \times 90$ Full Body Matrix approach, Newtonian mechanics was applied to all 15 rigid bodies and equations of motion were arranged in a $90 \times 90$ matrix such that inter-segmental joint reaction forces and moments were calculated simultaneously using an inverse matrix solution.

During comparative analysis of these three implemented approaches, the $90 \times 90$ matrix solution was determined to be the most comprehensive approach for dynamic modelling of the human body. It was more accurate and less time consuming as compared to the first two approaches, (i.e., the Linked Chain Segmental and Half Linked Segmented-Half Matrix approaches). This was largely due to the fact that with the Full Body $90 \times 90$ Matrix approach, experimental and numerical error gets equally distributed between all rigid body links rather than getting clustered at the terminating joint of the chain (i.e., the L5-S1 joint in the Linked Chain Segmental approach) during force and moment calculations. In addition, simultaneous calculation of all forces and moments is fast using the LU Decomposition in the Full Body $90 \times 90$ Matrix approach as compared to the Linked Chain Segmental and Half Linked Segmented-Half Matrix calculations. 


\subsubsection{Validation of the Multibody Dynamic Model}

Validation of the multibody dynamic model is necessary in order to check whether all translational and rotational dynamic equations are generated properly for accurate estimation of forces and moments of the 15 body segments. In other words, it assures the accuracy of the humanoid mathematical modelling strategy for three-dimensional kinematic analysis. Generally, T-pose data validation is carried-out by applying the following two steps.

\section{Static Case}

\section{Excluding Gravity Forces}

This validation step was based on the premise that if the gravity component of force for all the segments of the human body are excluded, the ground reaction forces at both feet should be exactly equal and opposite to each other. In other words, this validation step ascertains that when there is no force acting downwards due to the weight of the body, the forces exerted by the ground on the right foot should be equal and opposite to the forces exerted by the left foot on the ground in order to satisfy the equilibrium of the system.

To this end, the GRF components on the right foot, $F_{g r f_{x}}, F_{g r f_{y}}$, and $F_{g r f_{z}}$ were chosen to be $70.4 \mathrm{~N}, 370.0 \mathrm{~N}$, and $100.2 \mathrm{~N}$ respectively. The results for the ground reaction forces on the left foot were calculated to be $-70.3 \mathrm{~N},-370.5 \mathrm{~N}$, and $-100.2 \mathrm{~N}$ for $F_{g r f_{x}}, F_{g r f_{y}}$, and $F_{g r f_{z}}$ in the medio-lateral, vertical, and anterio-posterior directions respectively. Table 5.1 demonstrates the outcomes of validation step I, excluding gravity components of the forces. The results are as expected.

Including Gravity Forces

This is second standardized validation step which demonstrates that when there are just two forces acting on the multibody dynamic model (i.e., forces due to gravity 
Table 5.1: Validation I results

\begin{tabular}{ccc}
\hline \hline Forces & Right Foot GR Forces (N) & Left Foot GR Forces (N) \\
\hline$F_{x}$ & +70.4 & -70.3 \\
$F_{y}$ & +370.0 & -370.5 \\
$F_{z}$ & +100.2 & -100.2 \\
\hline
\end{tabular}

$\left(M_{\text {body }} g\right)$ acting downwards and ground reaction forces $\left(F_{G R F}\right)$ acting upwards) these two force contributions should be equally balanced with each other when all the inertia forces are absent. In this validation test, the gravity forces should be balanced by the ground reaction forces acting on both feet. In this case, when only the $M_{b o d y} \vec{g}_{\text {body }}$ component is considered (i.e., body weight) for all equations of motion of the whole body, the following requirement was supposed to be fulfilled:

$M_{\text {body }} g_{\text {body }}$ (acting downwards) $=G R F_{\text {rightfoot }}+G R F_{\text {leftfoot }}$ (acting upwards)

In our validation test, when the vertical component of the ground reaction force (GRF) at the right foot was $370.0 \mathrm{~N}$ in the vertical direction (+ve in the upwards direction), $+341.9 \mathrm{~N}$ was obtained for the left foot (acting upwards). Combined these sum to $711.9 \mathrm{~N}$ acting upwards. On the other hand, the gravity force component was:

Total mass of body $\left(M_{\text {body }}\right)=72.57 \mathrm{~kg}$ and $\mathrm{g}=[0 ;-9.81 ; 0]$. So, $M_{\text {body }} g=-711.9$ $\mathrm{N}$, which was acting downwards, equally balanced the force exerted by the ground on both the feet. Table 5.2 depicts the outcomes for validation step II.

Table 5.2: Validation II results

\begin{tabular}{ccc}
\hline \hline Forces & Ground Reaction Force(N) & Gravity Force (N) \\
\hline$F_{x}$ & 0 & 0 \\
$F_{y}$ & +711.9 & -711.9 \\
$F_{z}$ & 0 & 0 \\
\hline
\end{tabular}


Based on these numerical validation experiments, it was determined that the governing equations were correctly assembled. Validation in the dynamic sense could be best assessed experimentally using the redundant foot pressure measurements as will be discussed subsequently.

\section{Dynamic Case}

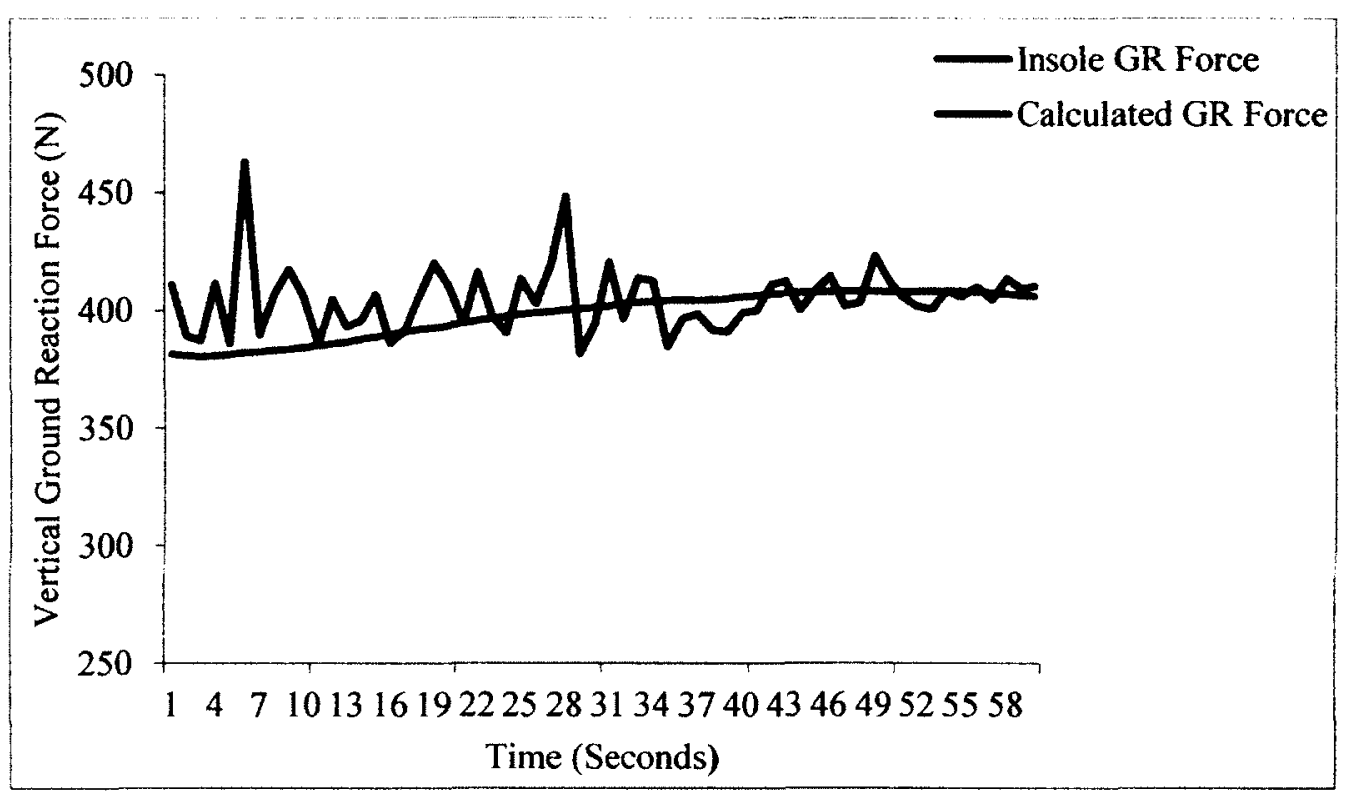

Figure 5.12: Vertical GRF force component from Insoles data and calculated from multibody dynamic model for dynamic validation

For dynamic validation, the vertical component of ground reaction force was considered. During experimental trials, the vertical GR force obtained from insoles was compared and plotted with the GR force calculated through the inultibody dynamic model computations. The main objective was to validate the dynamic characteristics of the developed model by comparing the experimental data values with the calculated outputs. Fig. 5.12 shows the vertical force component of insole data (for the left foot) and calculated left foot GR force component for 60 seconds time period. 
Both data outputs seem to be in pretty reasonable agreement during experimentation trials, which provides favourable dynamic validation results.

\subsubsection{Joint Work Calculations}

The final step of the multibody dynamic model development was to determine the mechanical work done by individual joints while maintaining postural stability during ship motion. For the 15 segment human model, there are a total of 14 linking points (i.e., joints) which must be considered for mechanical work measurements. Work done at each joint can be expressed as:

$$
W_{\theta_{1} \rightarrow \theta_{2}}=\int_{\theta_{1}}^{\theta_{2}} \vec{M}_{\text {joint }} \bullet d \vec{\theta}
$$

or

$$
W_{\Delta \theta}=\int_{\theta_{1}}^{\theta_{2}} \vec{M}_{j o i n t} \bullet d \vec{\theta}
$$

Here $W_{\theta_{1} \rightarrow \theta_{2}}$ is the amount of work performed by the joint in changing orientation from $\theta_{1}$ to $\theta_{2} ; \vec{M}_{\text {joint }}$ is the moment vector acting at that particular joint; and $d \vec{\theta}$ is incremental change of joint angle while maintaining stability of the segment. More-

over, since work is a scalar quantity, the mechanical work of the whole human body can be obtained by summation of the mechanical work performed by all 14 individual joints during a particular period of time such that:

$$
W_{\text {body }}=\sum\left(W_{\text {wrist }_{1}}+W_{\text {elbow }_{2}}+W_{\text {shoulder }_{3}}+W_{\text {head-neck }_{4}}+\ldots+W_{\text {ankle }_{14}}\right)
$$

Therefore, in the above equation (Eq. 65), $W_{\text {body }}$ is mechanical work performed by the whole human body while undergoing postural change with respect to changing ship motion. 


\section{Chapter 6}

\section{Results and Discussion}

\subsection{Introduction}

This chapter details various data processing and statistical analysis techniques that are used to analyze the experimental data that were collected as described in Chapter 4. The results obtained on the effect of ship motion on the energy consumption and mechanical work-metabolic energy correlation as well as other outcomes are discussed in this chapter.

\subsection{Methods}

In order to ascertain a statistically adequate number of subjects for significant validation of the outcomes, the average of metabolic energy data at one particular sea state were determined for increasing number of participants. Fig. 6.1 represents a typical result of the convergence of metabolic energy variations with the number of subjects. In this figure, bar 1 represents the metabolic energy data of one subject, while bar represents the average metabolic energy data of 2 subjects. It can be seen in Fig. 6.1, that metabolism levels are randomly varying upto 8 subjects, while the variation in the average of the metabolic energy consumption converges for 9 and 10 


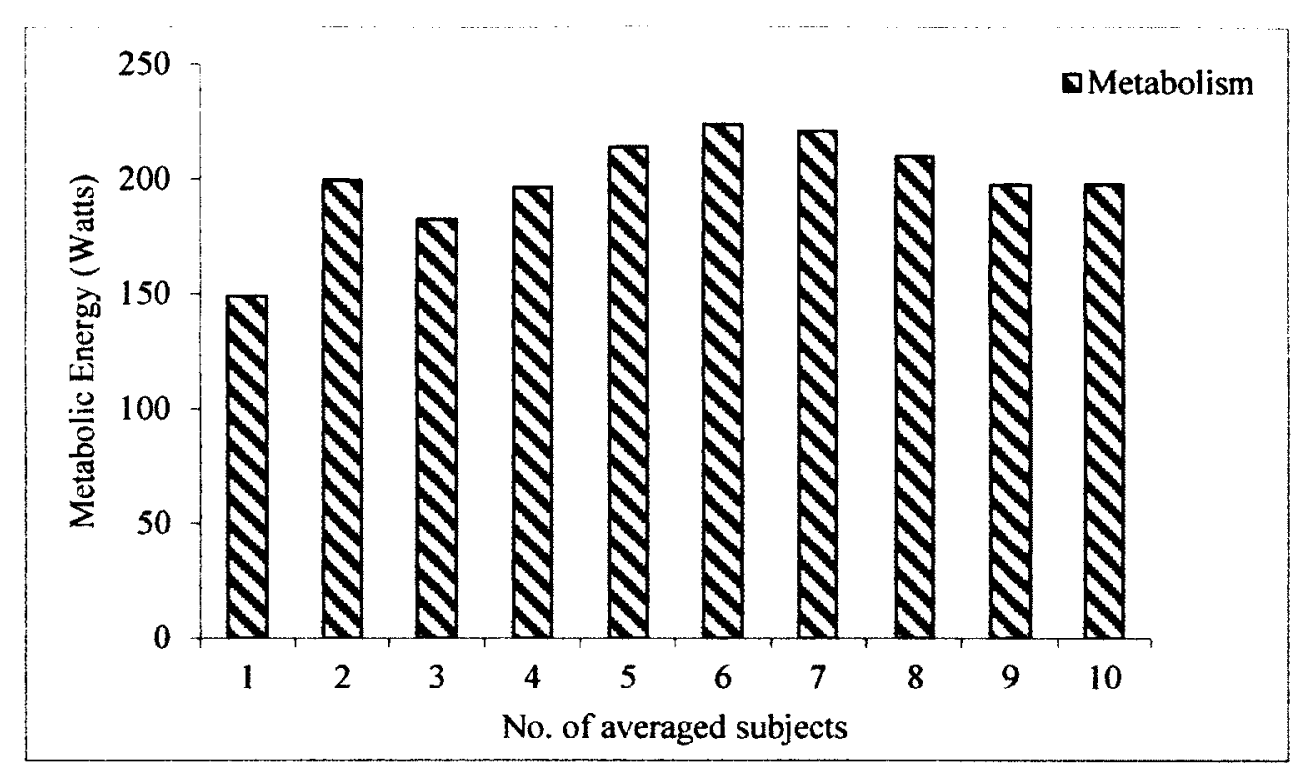

Figure 6.1: Convergence of metabolic levels on basis of no. of averaged subjects

subjects repectively. This indicated that results based on the data obtained from 10 subjects should be statistically reliable.

Different techniques were applied for analysis of this data. The metabolic energy data collected from ten subjects were interpolated using MATLAB and then averaged for the time period of 10 seconds to get the distributed energy expenditure. Fig. 6.2 shows the raw energy data (in watts) and interpolated and averaged data over 10 seconds obtained from the BB1LP respirometry system. This was necessary because the breath by breath system exports the energy data on a per breath basis which is nonuniformly distributed in time. The interpolated and averaged 120 data sets for 3 motion types were further averaged on sea state basis for metabolism variation with sea roughness outcomes. Moreover, metabolic energy results were categorized by male or female subject to compare the metabolic levels between the two genders. Metabolic data of 6 males and 4 females was considered for gender basis metabolism evaluation. The vertical ground reaction force (GRF) component was exported from 


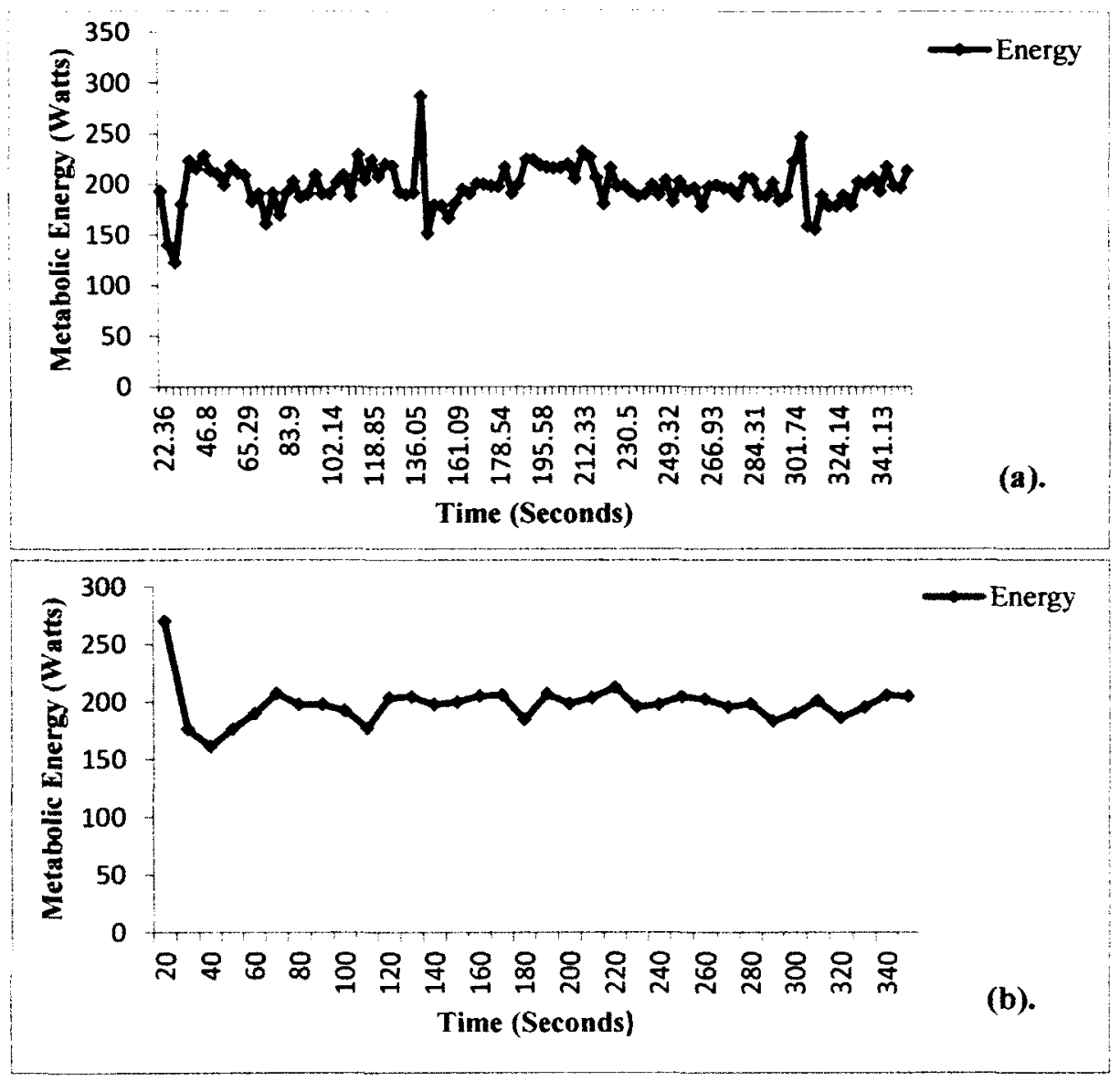

Figure 6.2: (a) Raw metabolic energy data; (b) Interpolated and averaged data

the load cell data directly by considering the axial forces. This was plotted with respect to simulated sea severity for 3 motion types in order to observe the effect of increasing motion perturbations on the force exerted by the ground on the feet for postural stabilization. The mechanical work done by the human body as well as 14 different body joints were calculated using the multibody dynamic model developed in Chapter 5 . Since the mechanical work consists of both positive and negative values, the absolute mechanical work was considered and averaged on 15 second intervals. The metabolic energy values were also interpolated using MATLAB and averaged over 15 seconds (it should be noted that mechanical work and metabolic energy data 
was averaged over 15 seconds scale for Pearson correlation). An outlier removal treatment was applied to the data prior to correlation testing, where 2-4 outliers were ignored for data accuracy. The mechanical work of the body was correlated with the corresponding metabolic energy expenditures by using the Pearson correlation technique. The mechanical work contributed by each of the 14 joints calculated from the multibody dynamic model was plotted with respect to each of the twelve motion states. This was done to identify the joints that performed the maximum mechanical work in response to perturbations. Appendix A shows the results of one particular subject's data sets, which are similar for all other subjects, who participated in data collection.

\subsection{Variation of Metabolic Energy Levels with Dif- ferent Ship Motions}

\subsubsection{Metabolic Energy Variation with Combined (PRY) Ship Motion}

As shown in Fig. 6.3, metabolic energy levels increase as the sea motion severity increases. The least severe and most severe energy levels can be clearly distinguished as metabolic levels are $11 \%$ greater in the most severe sea state when compared to the least severe sea state. Energy levels in sea state 2 are only $1 \%$ higher than in sea state 1, while for the next successive states, energy levels elevate by approximately $5 \%$ for severe (state 3 ) and very severe states (i.e., sea state 4), respectively. 


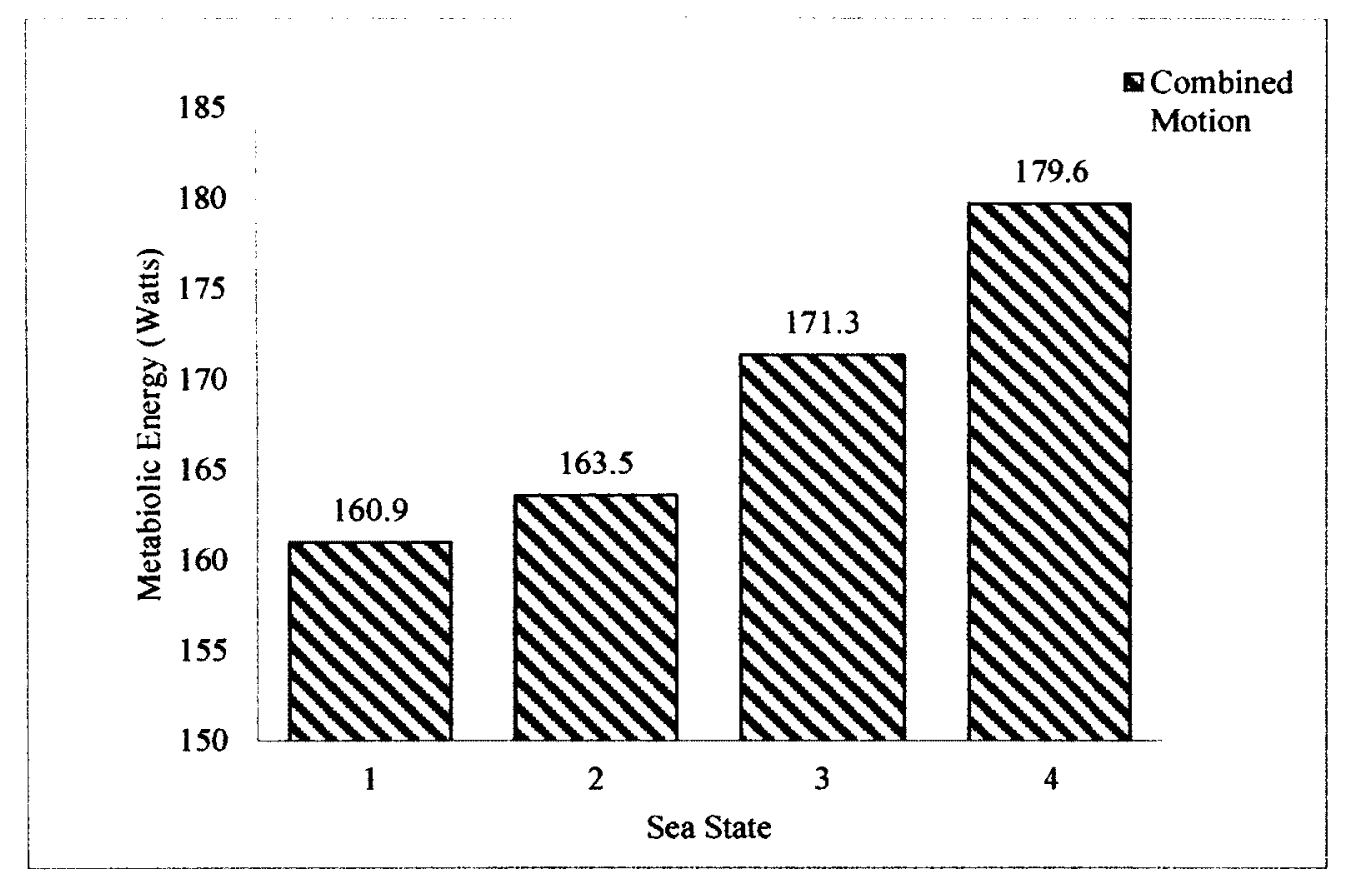

Figure 6.3: Energy variation with combined (PRY) motion

\subsubsection{Metabolic Energy Variation with Roll Motion}

In the case of roll motion (Fig. 6.4), the energy levels also increase with increasing sea state. Sea state 4 has energy levels less than $1 \%$ higher than sea state 3 . So, these two sea states cause similar energy expenditure levels. Metabolic levels in sea state 2 are $2 \%$ higher as compared to sea state 1 . Further, metabolic energy increases by approximately $3 \%$ from the medium sea state (sea state 2 ) to severe and very severe states (sea states 3 and 4 ). The energy levels for the most severe state (i.e., sea state 4) are $5 \%$ greater than the least severe sea state (sea state 1).

\subsubsection{Metabolic Energy Variation with Pitch Motion}

As shown in Fig. 6.5, in the case of pure pitch motion, the most severe energy level (sea state 4) results in approximately $5 \%$ greater energy expenditure than the least 


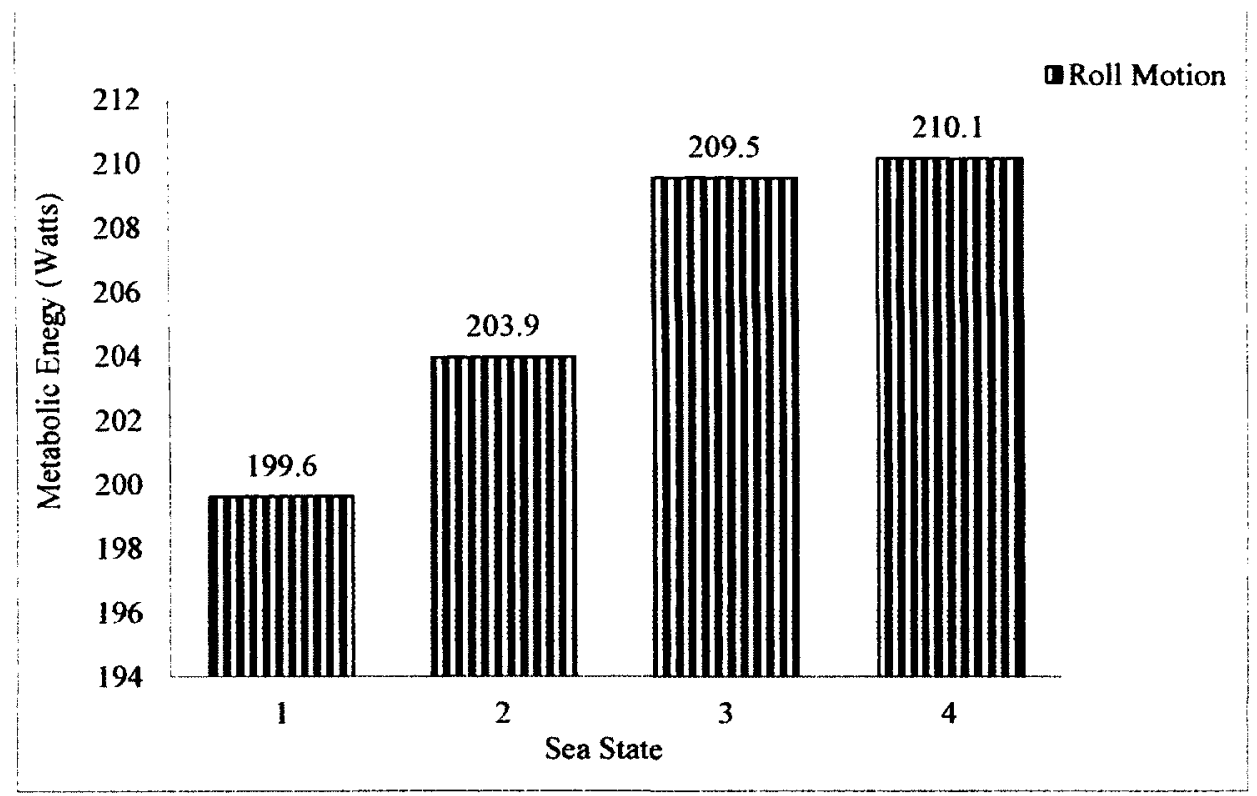

Figure 6.4: Energy variation with roll motion

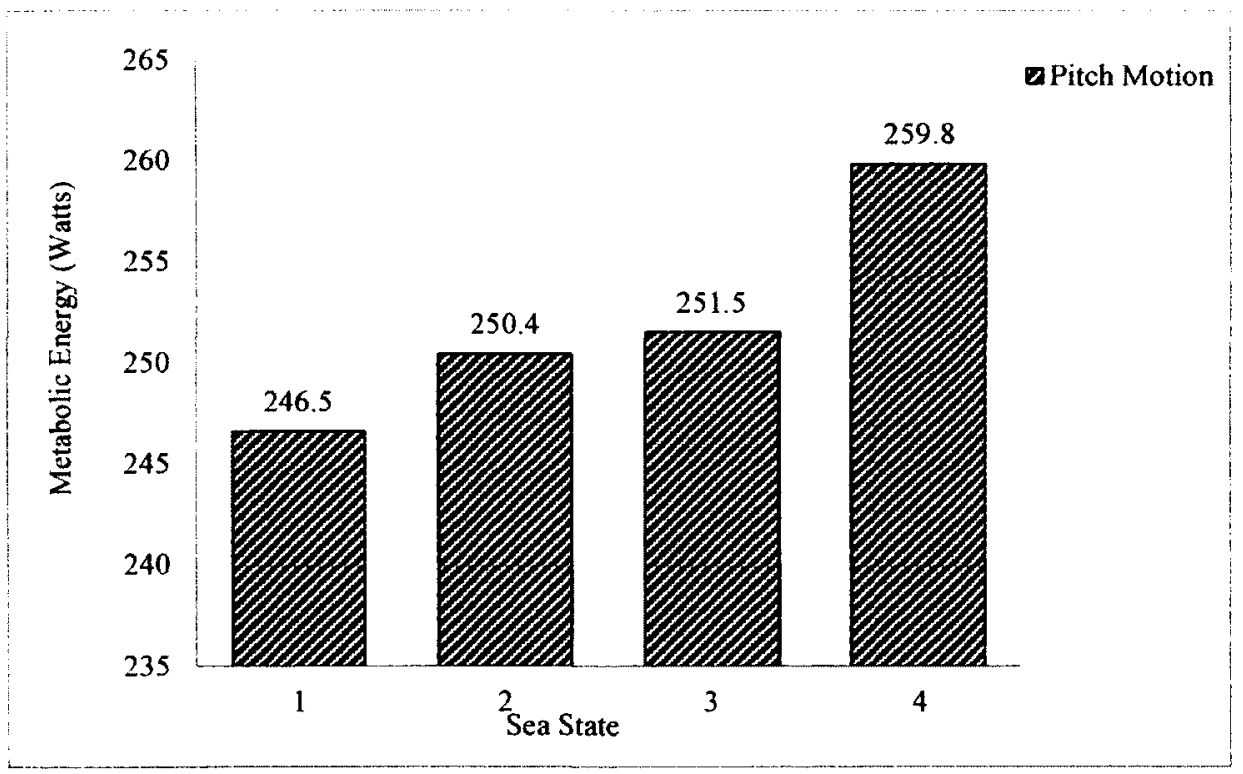

Figure 6.5: Energy variation with pitch motion 
severe sea state (sea state 1). Metabolic energy consumption follows the upward trend as the severity increases; however the metabolic energy for sea states 2 and 3 are similar with a metabolic difference of less than $1 \%$ between the two states. As the sea state becomes very severe (sea state 4 ), the metabolism is increased by $3 \%$ from the previous state (sea state 3 ).

\subsubsection{Comparative Analysis for Metabolic Energy Levels}

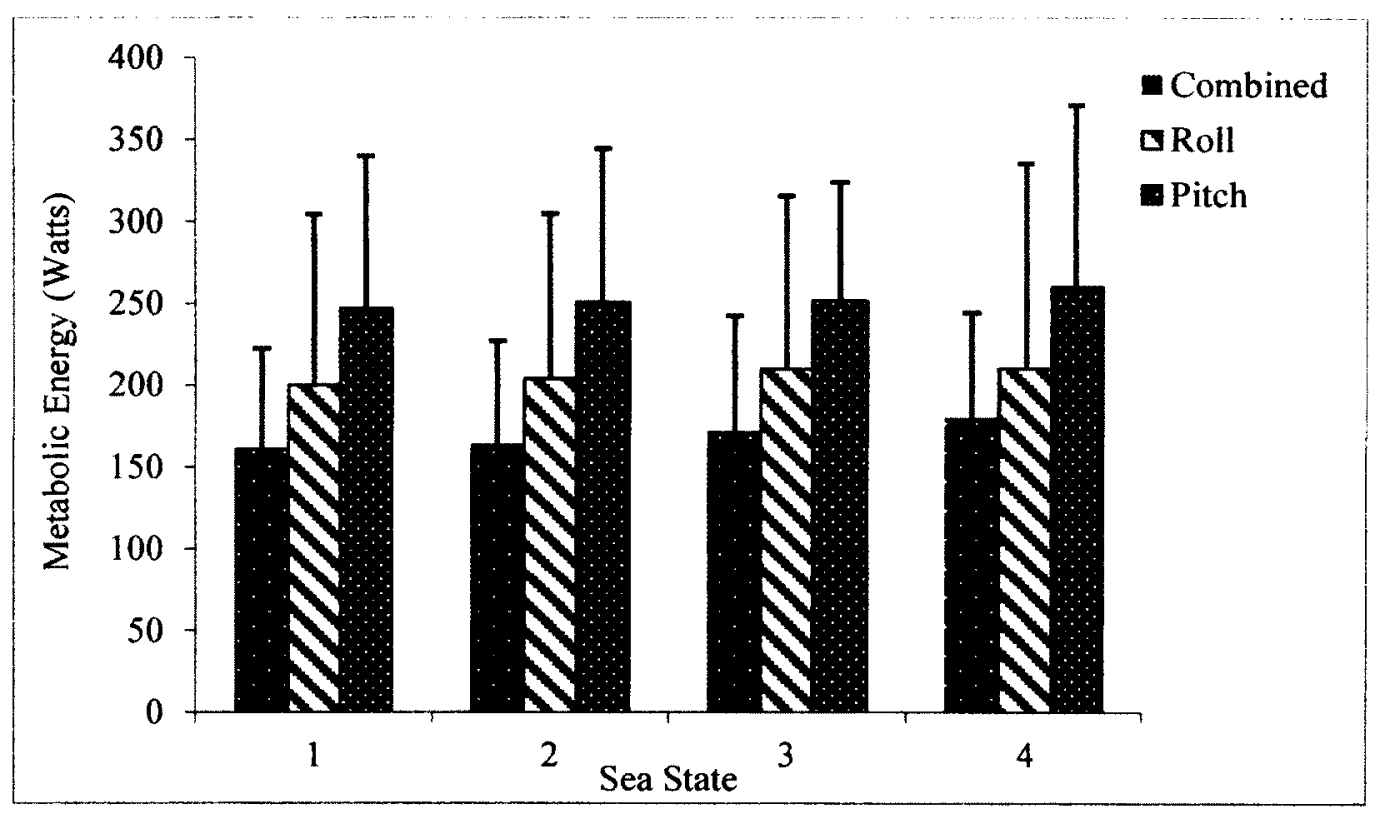

Figure 6.6: Metabolic Energy (Std.) with motion severity for combined (PRY), roll, and pitch motion

The mean metabolic energy levels with corresponding standard deviations for all subjects during the three types of ship motion (combined, roll, and pitch) for all twelve motion states are plotted in Fig. 6.6. For sea state 1, the energy levels of combined, roll, and pitch cases are $160.9,199.6$ and $246.5 \mathrm{~W}$ respectively, where energy for general/combined ship motion is least which is comparable to resting state 
metabolic levels. Fig. 6.6 indicates that, for example, at sea state 1, the metabolic energy consumption for pitch motion is approximately $23 \%$ higher than that for roll motion, which in turn requires an energy level $24 \%$ higher than general or combined motion. As already shown in Figures 6.3, 6.4, and 6.5, metabolic levels for the most severe sea state for all motion types are comparable. The pitch motion has the highest levels of metabolic energy consumption $(259.8 \mathrm{~W})$ in the most severe sea state (sea state 4$)$, which is greater than roll energy severity levels $(210.1 W)$. Both roll and pitch metabolic energy levels are significantly higher than the combined (PRY) motion levels $(179.6 \mathrm{~W})$ for sea state 4 . The metabolic consumption rate during sea state 4 was $23 \%$ greater in pitch motion as compared to roll motion. Further, the roll motion consumes $16 \%$ more energy than the combined case (PRY) in this sea state. Therefore, Figure 6.6 indicates that pitch motion has a greater amount of energy expenditure as compared to the other two types of motion (roll and combined) and combined motion requires the least energy consumption in all sea states. Standard deviation values vary between $100.3-125.2 W$ for sea state 1 and state 4 for roll motion respectively. The standard deviation values for roll motion are higher as compared to pitch motion, which lie in the range of $72.2-111.2 \mathrm{~W}$. It shows that metabolic energy levels in roll motion are dispersed more from the average as compared to pitch motion. While the standard deviation for the combined ship motion is least (i.e., 61.2-70.8 $W$ ) of all three motion types.

\subsubsection{Male-Female Metabolic Energy Variation with Motion}

As shown in Fig. 6.7, females undergo lesser values of maximal $\mathrm{O}_{2}$ consumption than males. This shows that the mechanical work performed by a female to stabilize herself is less than the work performed by a male. The male energy levels are $30 \%$ and $51 \%$ greater than females in pitch and combined motions, respectively. While in the case of roll motion, energy levels between sexes differ more significantly, as males used 


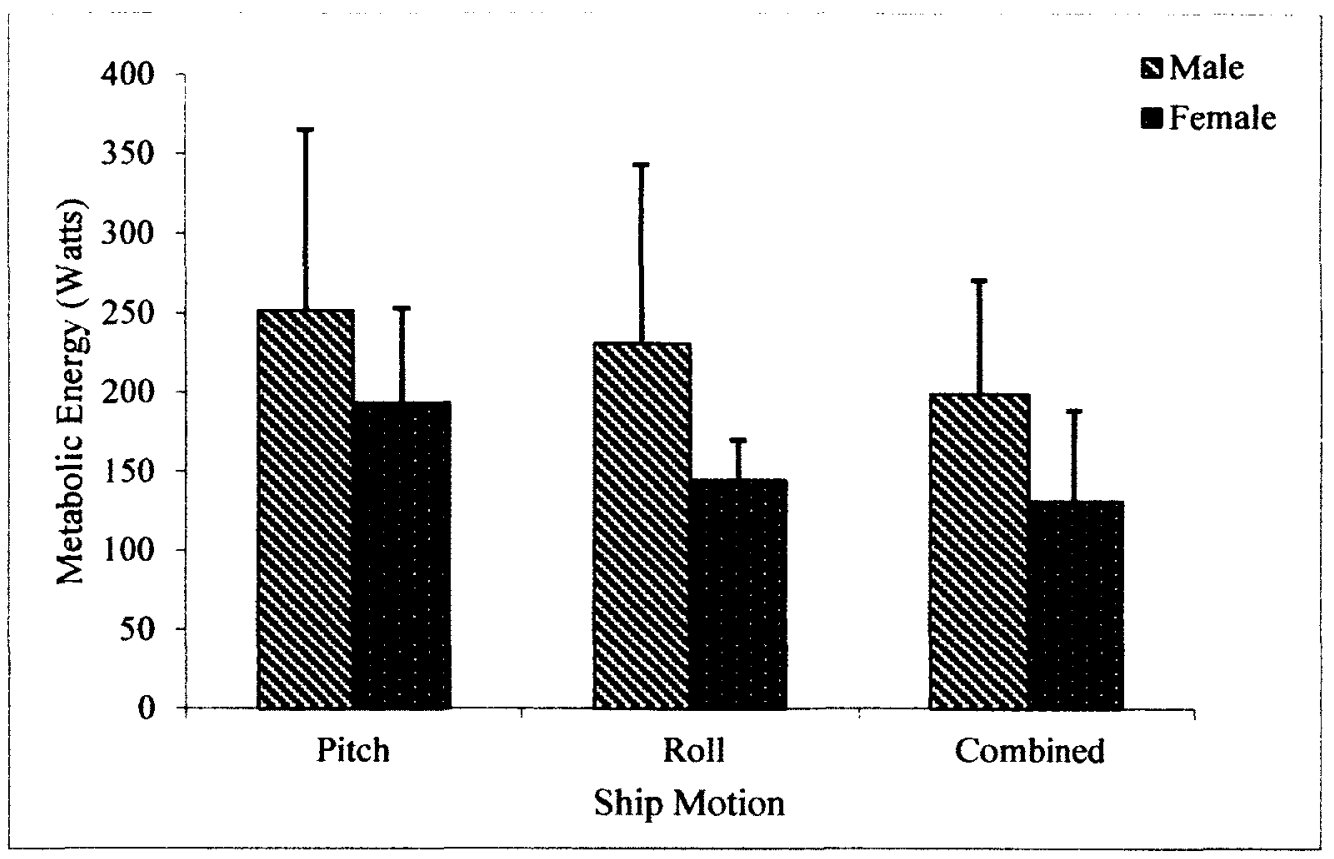

Figure 6.7: Mean (Std.) metabolism for male and female factor

approximately $59 \%$ greater energy than females.

The standard deviation for males is higher for pitch and roll motions (i.e., 113.6 and $112.8 \mathrm{~W}$ respectively) as compared to combined ship motion (i.e., $64.3 \mathrm{~W}$ ). Females also have standard deviation values higher in pitch motion than roll and combined motion. Further, for roll motion, female metabolic levels are dispersed with the least variation from the mean values as the standard deviation is lowest (i.e., $23.4 \mathrm{~W}$ ). 


\subsection{Different Body Joint Contributions to Main- tain Postural Stability of the Human Body}

\subsubsection{Mechanical Work Performed by Individual Joints in Combined Ship Motion}

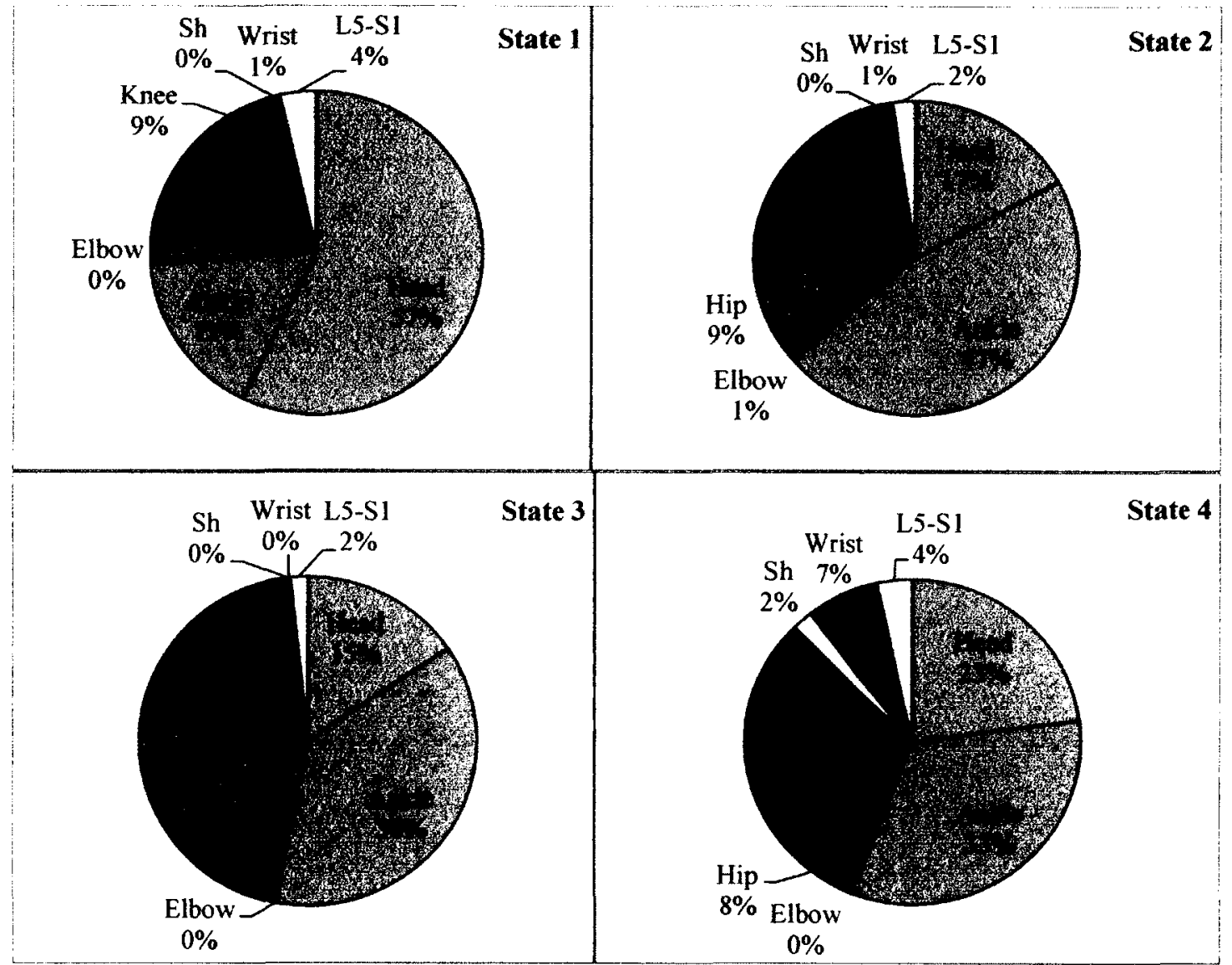

Figure 6.8: Joint mechanical work contribution for all sea states in combined motion: (a) 1 meter waves (least severe); (b) 2 meter waves (medium); (c) 5 meter waves (severe); (d) 7 meter waves (most severe)

Experimental data of 10 subjects was considered for analysis of joint workload for combined motion during different sea states. Fig. 6.8 shows the mechanical work 
performed by various joints in four sea states of combined motion to maintain postural stability. It can be seen that the mean joint work done by the lower extremities is consistently higher with more severe ship motion. For the least severe combined motion (Fig. 6.8a) (i.e., 1 meter waves), the work done by all joints is randomly distributed and focussed mainly in the head-neck joint (i.e., 57\%). Ankle, knee, hip, and L5-S1 joints also contribute $17 \%, 9 \%, 12 \%$, and $4 \%$ respectively. This is because the corresponding ship motion is very similar to a rest standing position. As the severity of ship motion increases, the lower extremity joints follow predominantly the ankle strategy phenomenon. The ankle strategy states that when the human body is subjected to motion perturbations (usually less severe), the ankle joints play a predominant role to maintain the mass centre of the body within the base of support (BoS). In addition to ankle joints, other lower extremity joints such as the knee, hip, and L5-S1 joints also play an important role in postural stability. However during less severe motions, the L5-S1 joint contributes less to stability maintenance. The head-neck joint is another predominant joint which plays an equally important role to the ankle joint. This is due to the visual feedback of the central nervous system that is provided in response to perturbations. Every time the human body is subjected to a motion perturbation, the head accelerations increase as the person tries harder to maintain postural stability by changing his/her stance width according to their visual acuity (alertness). Moreover, as the motion becomes more and more severe, the involvement of other body joints in maintaining the postural stability increases. For instance, by comparing Figures $6.8 b$ with $6.8 d$, the mechanical work contribution by joints of the upper extremity (i.e., shoulder, elbow, and wrist joints) become significant as the ship motion becomes more severe. In Fig. $6.8 b$, the wrist and elbow joints contribute $1 \%$ and the shoulder joint has no contribution, while in the most severe state (Fig. 6.8d), the shoulder becomes a significant joint producing $2 \%$ and the wrist joint is showing $7 \%$ mechanical work contribution. The head-neck joint always shows 
significant mechanical work for all motion states. In the least severe state, head accelerations are very high showing 57\% mechanical work while for higher sea states, these are almost constant by varying their contribution between approximately $15 \%-23 \%$. Further, the joint mechanical work distribution has been more oriented towards the mixed strategy (i.e., hip and ankle strategy) with the severe perturbations. Fig. $6.8 c$ shows that the hip joint is more active in sea state 3 and contributes $24 \%$ as compared to sea state 2 (where it contributes $9 \%$ ). These outcomes for the body joint mechanical energy distribution for postural stability are in accordance with the ankle and hip strategy patterns defined by Nashner [74]. Moreover, the results from Fig. 6.8d show that the ankle joint is still actively involved along with other lower extremity joints by contributing $33 \%$ in order to maintain stability during rapid perturbations. These results are consistent with the joint torque findings which show that the pure hip strategy (by eliminating ankle joint mechanical work) is not sufficient, for postural stability maintenance during rapid ship motions [74]. This is because during fast and rapid perturbations, torque produced by hip flexion is additive to ankle plantar flexor torque, which together maintain the stabilized position of the human body with rapid perturbations. In addition, the knee joint performs almost the same mechanical work in Figures $6.8 b, c$, and $d$ motion states by contributing approximately $23 \%$. While work done by the L5-S1 joint increases from $2 \%$ to $4 \%$ as sea severity increases (Fig. $6.8 b$ and $d)$.

\subsubsection{Mechanical Work Performed by Individual Joints in Roll Motion}

Similarily, the experimental data of 10 subjects were considered for joint workload analysis for roll motion. As in the case of combined motion, the least severe roll motion state (Fig. 6.9a) resembles a stand still position where the mechanical work 


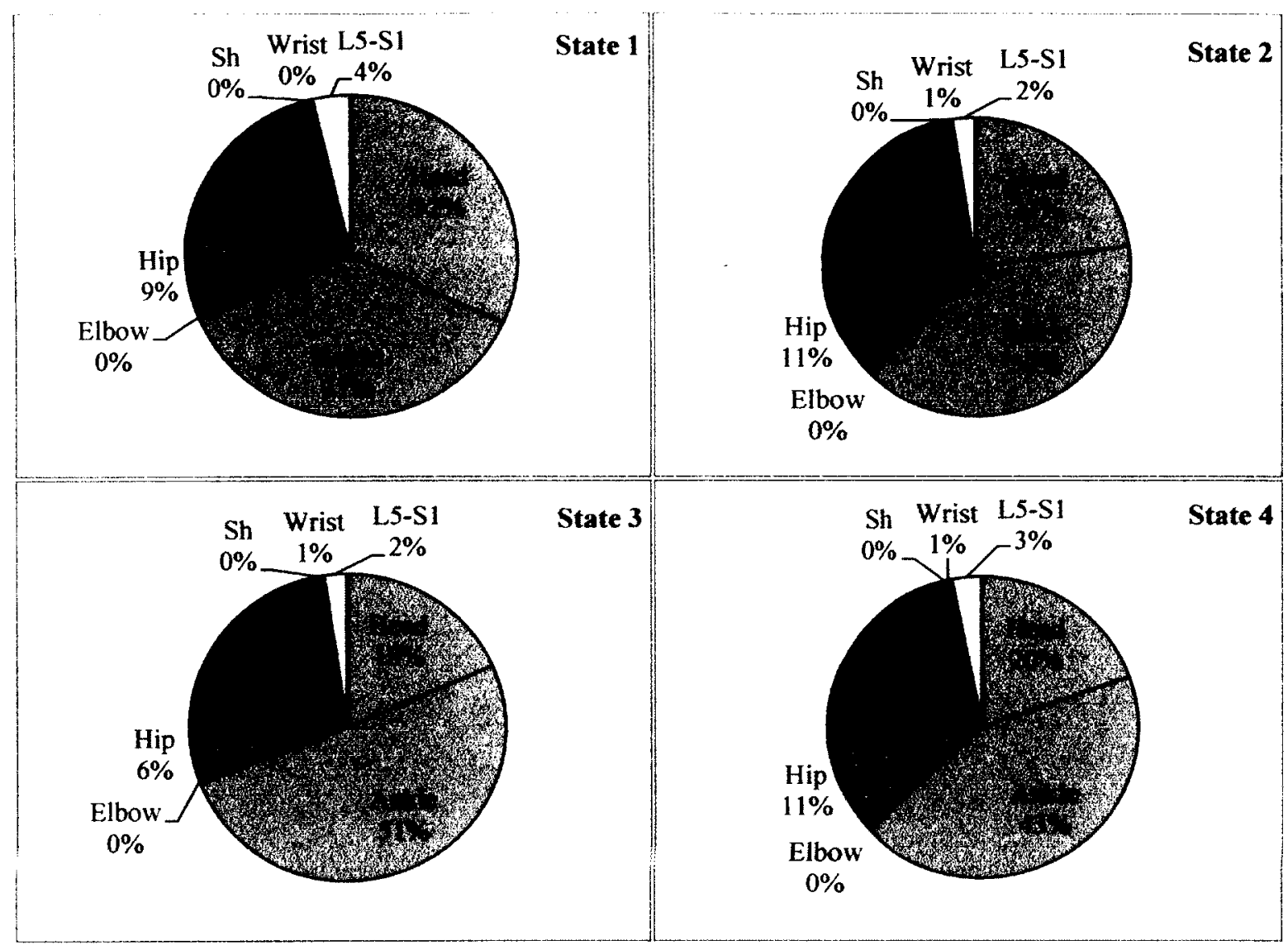

Figure 6.9: Joint mechanical work contributions for all sea states in roll motion: (a) 1 meter waves (least severe); (b) 2 meter waves (medium); (c) 5 meter waves (severe); (d) 7 meter waves (most severe)

is equally distributed throughout all the joints. In sea state 2 , the postural sway is more significant for ankle joints by activating ankle plantar flexor translations and rotations as stability efforts begin. Fig. $6.9 b$ shows the major activation of ankle joints which contribute approximately $39 \%$ for stability maintenance. Moreover, in all the sea states (i.e., Fig. $6.9 b, c$, and $d$ ), the knee joints become significantly more involved in order to produce torque by contributing $22 \%-24 \%$ to the total mechanical work. The hip joint contribution increases in state 4 by generating $11 \%$ work as compared to the previous state (Fig. $6.9 \mathrm{c}$ ) where the contribution is just $6 \%$. In addition to this, 
torque production in the lumbar-sacral region (i.e., L5-S1 joint) increases slightly as the sea state becomes more severe. For instance, the L5-S1 contribution is $2 \%$ in states 2 and 3 , but reaches $3 \%$ in sea state 4 . The head-neck joint mechanical work is always significant in every sea state, although it appears to decrease in more severe sea states. In Fig. 6.9b, head accelerations contribute to $23 \%$ which decrease to $18 \%$ and $20 \%$ respectively as the perturbations become more rapid (i.e., Figures $6.9 c$ and $6.9 d)$. Thus in response to roll motion, the overall joint mechanical work follows the phenomenon of including more body joints for mechanical work generation by activating a greater number of muscles within the body during an increase in the motion amplitude and frequency.

\subsubsection{Mechanical Work Performed by Individual Joints in Pitch Motion}

In the pitch motion as well, the data was plotted for 14 joints considering four different sea states. For the least severe case (Fig. 6.10a), the mechanical work is equally distributed throughout the joints in order to maintain stability. This is due to the fact that the body is more likely to be in stand still posture rather than actually generating any muscle tension at the particular joint for stability (i.e., head-neck, ankle, hip, knee, and L5-S1 joints contribute $24 \%, 30 \%, 18 \%, 14 \%$, and $12 \%$ respectively). As the severity increases, more mechanical work is carried out by the lower extremity joints, while the work done by the upper extremity joints, (i.e., shoulder, elbow, and wrist joints) is almost insignificant as in combined motion. For example in Fig. $6.10 b$ (i.e., 2 meter waves), the ankle, knee, and head-neck joints are predominant ones by contributing approximately $39 \%, 24 \%$, and $27 \%$, respectively, whereas the L5-S1 and hip joints are minority contributors with $4 \%$ and $5 \%$ respectively. As the motion severity increases, the contributions from the upper joints become more significant. 
In fact, the upper extremity mechanical work is very significant in the sea state 3 while in the sea state 4 , joint torque is more focussed in the ankles, knees, and hips for implementation of the mixed strategy to maintain stability. For example, in Fig. $6.10 d$, the ankle, knee, and hip joints are the most active joints by contributing total mechanical work of $77 \%$ where the hip joint mechanical work contribution increases from $5 \%$ to $15 \%$ as the motion state changes from 2 to 4 (Fig. $6.10 b$ to $d$ ). Head-neck torque with approximately $17 \%$ is also significant for maintaining a stabilized position by making recurrent head movements due to instability alertness. The lumbar-sacral

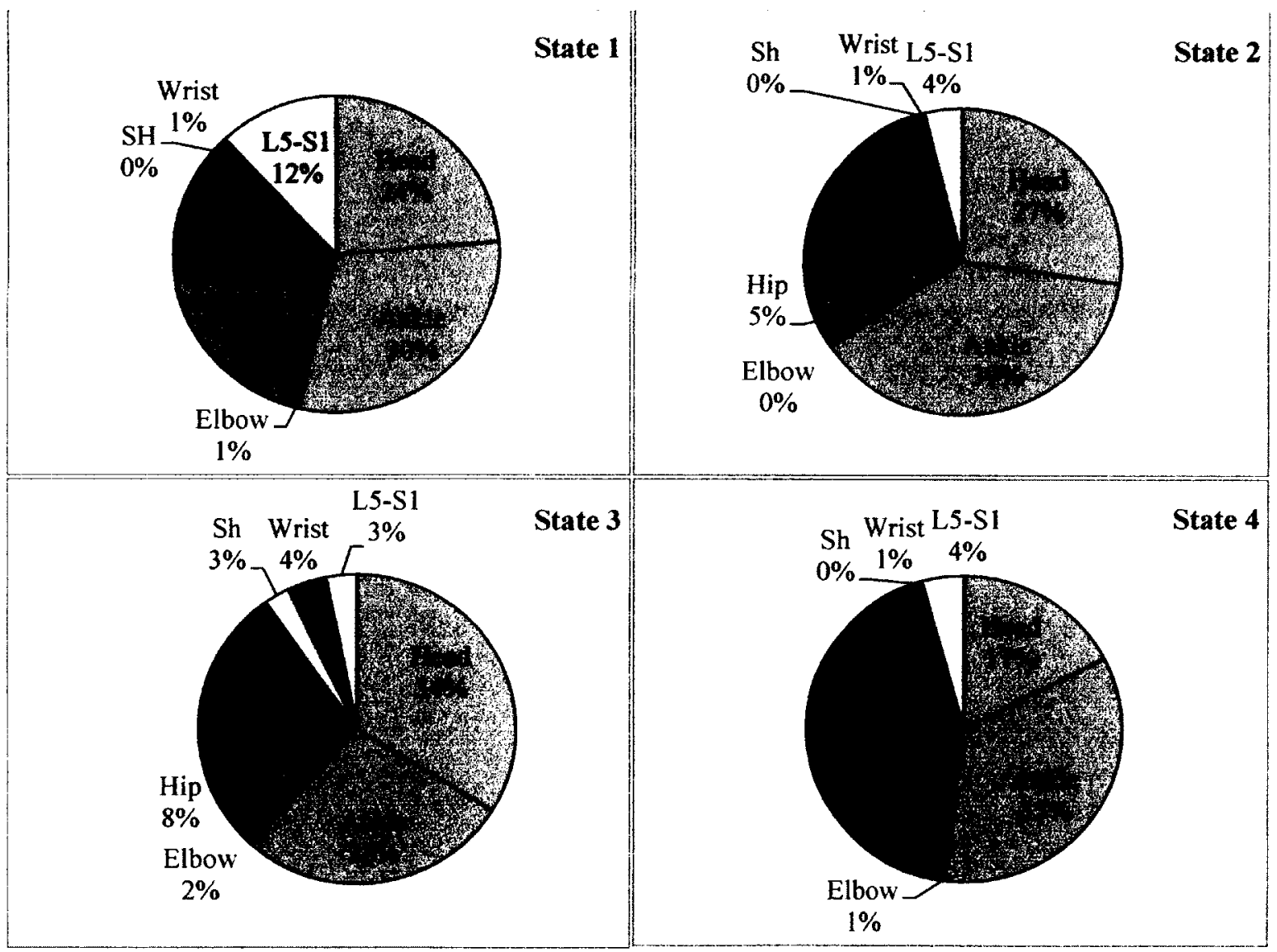

Figure 6.10: Joint mechanical work contributions for all sea states in pitch motion: (a) 1 meter waves (least severe); (b) 2 meter waves (medium); (c) 5 meter waves (severe); (d) 7 meter waves (most severe) 
joint work contribution also increases as severity increases. For example, the L5-S1 joint is contributing $3 \%$ in sea state 3 (Fig. $6.10 \mathrm{c}$ ), but increases its contribution to $4 \%$ in sea state 4 (Fig. 6.10d).

\subsection{Effect of Ship Motion Severity on Vertical Ground Reaction Force}

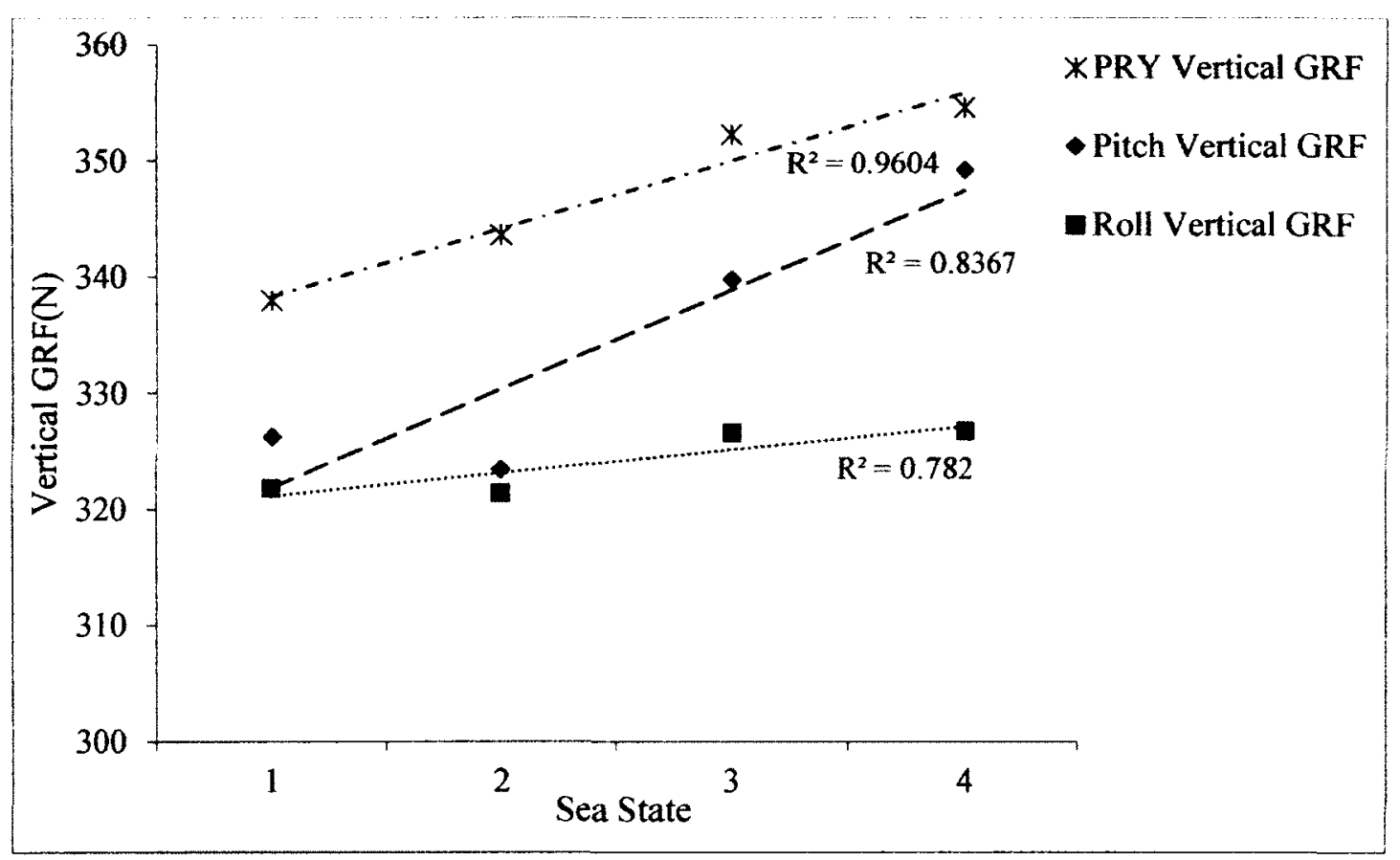

Figure 6.11: Vertical ground reaction force component variation with ship motion

The results in Fig. 6.11 indicate that the vertical component of ground reaction force (GRF) for all types of motions increases with the sea severity. For combined motion, the GRF component in sea state 1 is $337.9 \mathrm{~N}$ which increases to $354.5 \mathrm{~N}$ in sea state 4. For the pitch motion, the increase in GRF appears to be significantly higher as compared to the other two motion types. However, the pitch GRF component decreases (i.e., $323.4 \mathrm{~N}$ ) in sea state 2 as compared to sea state 1 (i.e., $326.2 \mathrm{~N}$ ). For 
higher sea states, the pitch GRF components increase as severity increases (i.e., 349.2 $N$ for sea state 4). In the case of roll motion, the GRF component reaches 326.7 $\mathrm{N}$ from $321.7 \mathrm{~N}$ with sea state 4 from state 1 . The GRF component of roll motion increases least significantly as compared to pitch and combined motions. The overall vertical GRF component difference between the least severe motion state (sea state 1) and most severe ship motion state (sea state 4) for combined, pitch, and roll motions can be easily distinguished with levels of $4 \%, 7 \%$, and $1 \%$ respectively.

\subsection{Work-Energy Correlation Results}

Using Pearson correlation, the calculated total mechanical work of the body correlated quite significantly with the corresponding measured expended metabolic energy. Table 6.1 shows the Pearson correlation coefficient values for each of the 10 subjects during all sea states. Significant correlation p-values vary from 0.420 to 0.988 for all 120 data sets. However, there exist a few data sets which show little or no correlation at all between the two variables. These were not considered for average and standard deviation calculations. Some of the p-values are missing in Table 6.1, as there were a few data-sets, which could not be analysed properly due to missing data points even after data editing. Overall, the average p-values lie in the range $0.6740-0.7835$ with standard deviations in the range 0.0809-0.1831 for the work-energy data sets. These are highly significant indicating that both variables are strongly correlated. 
Table 6.1: Pearson correlation coefficients between mechanical work and metabolic energy consumption for subject data

\begin{tabular}{|c|c|c|c|c|c|c|c|c|c|c|c|c|}
\hline Sub. No. & $P_{1}$ & $P_{2}$ & $P_{3}$ & $P_{4}$ & $R_{1}$ & $R_{2}$ & $R_{3}$ & $R_{4}$ & $P R Y_{1}$ & $P R Y_{2}$ & $P R Y_{3}$ & $P R Y_{4}$ \\
\hline 1 & 0.6338 & 0.6204 & 0.7480 & 0.6956 & 0.988 & 0.7650 & 0.7485 & 0.8356 & - & 0.5922 & 0.4786 & 0.5854 \\
\hline 2 & 0.8311 & 0.9300 & 0.9171 & 0.8119 & 0.8763 & 0.7974 & 0.9190 & 0.9361 & 0.9805 & 0.6207 & 0.8158 & 0.7082 \\
\hline 3 & 0.7019 & 0.5683 & 0.7195 & 0.8554 & 0.6737 & 0.5463 & 0.7129 & 0.7179 & 0.5828 & 0.7355 & $0.1867 *$ & 0.8202 \\
\hline 4 & 0.6879 & 0.6185 & 0.6922 & 0.9041 & 0.9013 & 0.6689 & 0.8630 & 0.7616 & 0.8342 & - & 0.5859 & 0.7843 \\
\hline 5 & 0.7880 & 0.7901 & 0.6088 & 0.7526 & 0.8181 & 0.8200 & 0.7855 & 0.6543 & 0.7026 & 0.7618 & 0.8994 & 0.6794 \\
\hline 6 & 0.6489 & 0.7592 & 0.5497 & 0.7071 & 0.6156 & 0.8512 & 0.8503 & 0.7950 & 0.7070 & 0.6815 & 0.6572 & 0.9786 \\
\hline 7 & 0.8224 & 0.7557 & 0.5124 & 0.6617 & - & 0.7047 & 0.6408 & 0.3266 & 0.8176 & 0.7126 & 0.8642 & 0.7089 \\
\hline 8 & 0.7925 & 0.7662 & 0.8483 & 0.8094 & 0.5205 & 0.7436 & - & 0.6530 & 0.5433 & 0.8263 & 0.7896 & 0.8041 \\
\hline 9 & 0.5400 & 0.5013 & 0.7691 & 0.7316 & - & - & 0.7210 & 0.6174 & $0.0351 *$ & - & - & 0.6397 \\
\hline 10 & 0.6165 & 0.4312 & 0.5267 & $0.2427 *$ & 0.7398 & 0.4201 & 0.8112 & - & 0.7322 & $0.045 *$ & $0.1886 *$ & 0.5074 \\
\hline Avg. & 0.7063 & 0.6740 & 0.6891 & 0.7172 & 0.7666 & 0.7020 & 0.7835 & 0.7463 & 0.7375 & 0.7043 & 0.7272 & 0.7216 \\
\hline Std. & 0.09876 & 0.1517 & 0.1380 & 0.1831 & 0.1579 & 0.1396 & 0.08688 & 0.1077 & 0.1407 & 0.0809 & 0.1564 & 0.1332 \\
\hline
\end{tabular}

$P_{i}$ - Pitch Motion at $i^{\text {th }}$ sea state $; R_{i}$ - Roll Motion at $i^{\text {th }}$ sea state $P R Y_{i}$ - Pitch/Roll/Yaw Motion at $i^{\text {th }}$ sea state ;

* - Data sets ignored during averaging; 


\subsection{Direct Comparison of Mechanical Work with Metabolic Energy}

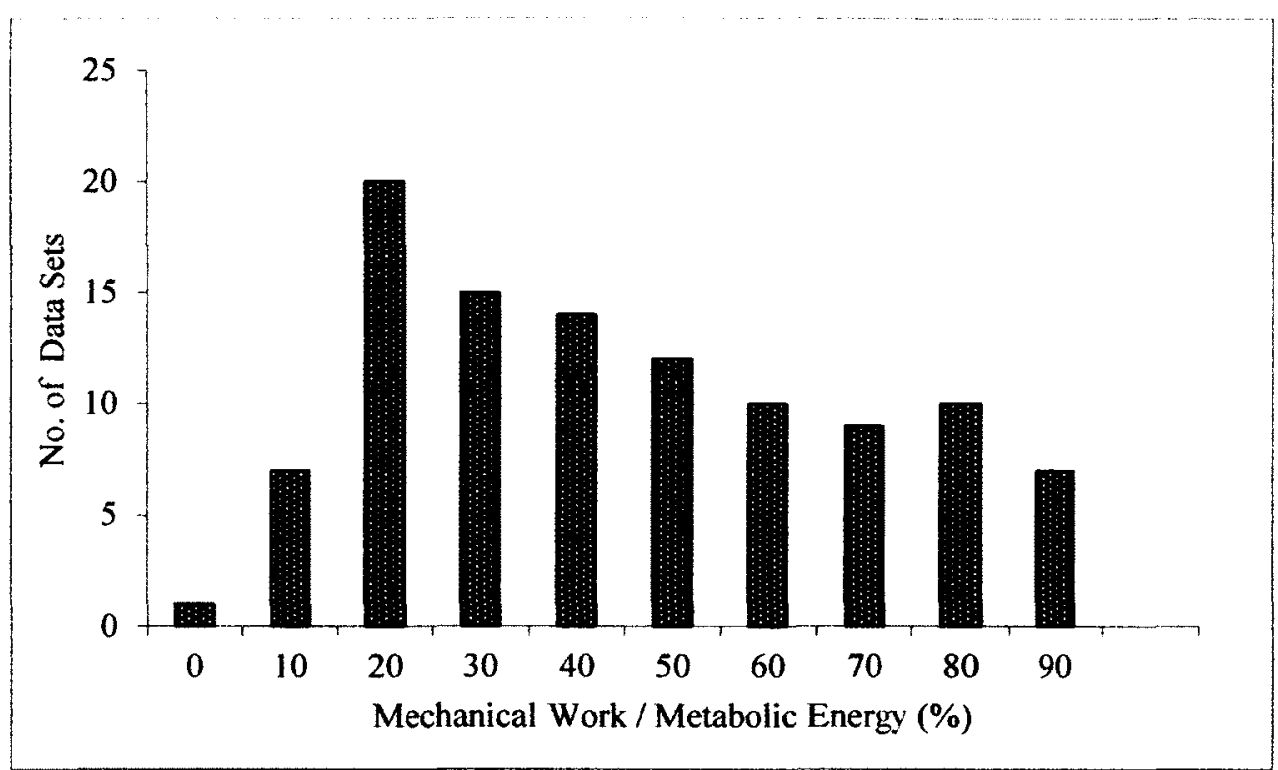

Figure 6.12: Mechanical work/Metabolic energy (\%age) with no. of data sets

The data sets for all participants and motion conditions were taken into consideration and ratio of the rate of mechanical work to the total metabolic energy expenditure was computed and presented as a percentage. There were total of 120 data sets, out of which 105 data sets were considered for this analysis. Fig. 6.12 shows the variation of the number of data sets with work/energy ratio for the 105 data sets. The figure shows the peak of the hump between $15 \%$ and $25 \%$ on the horizontal scale and showing that 20 data sets lie in this range. The ratio remains in the range $15 \%-50 \%$ for most of the data sets. There is only one data set for which the work/energy ratio is below $5 \%$ (in other words, where only $5 \%$ of the metabolic energy expended is attributable to mechanical work). There are also 17 data sets 
where mechanical work accounts for $75 \%-95 \%$ of the metabolic energy expended.

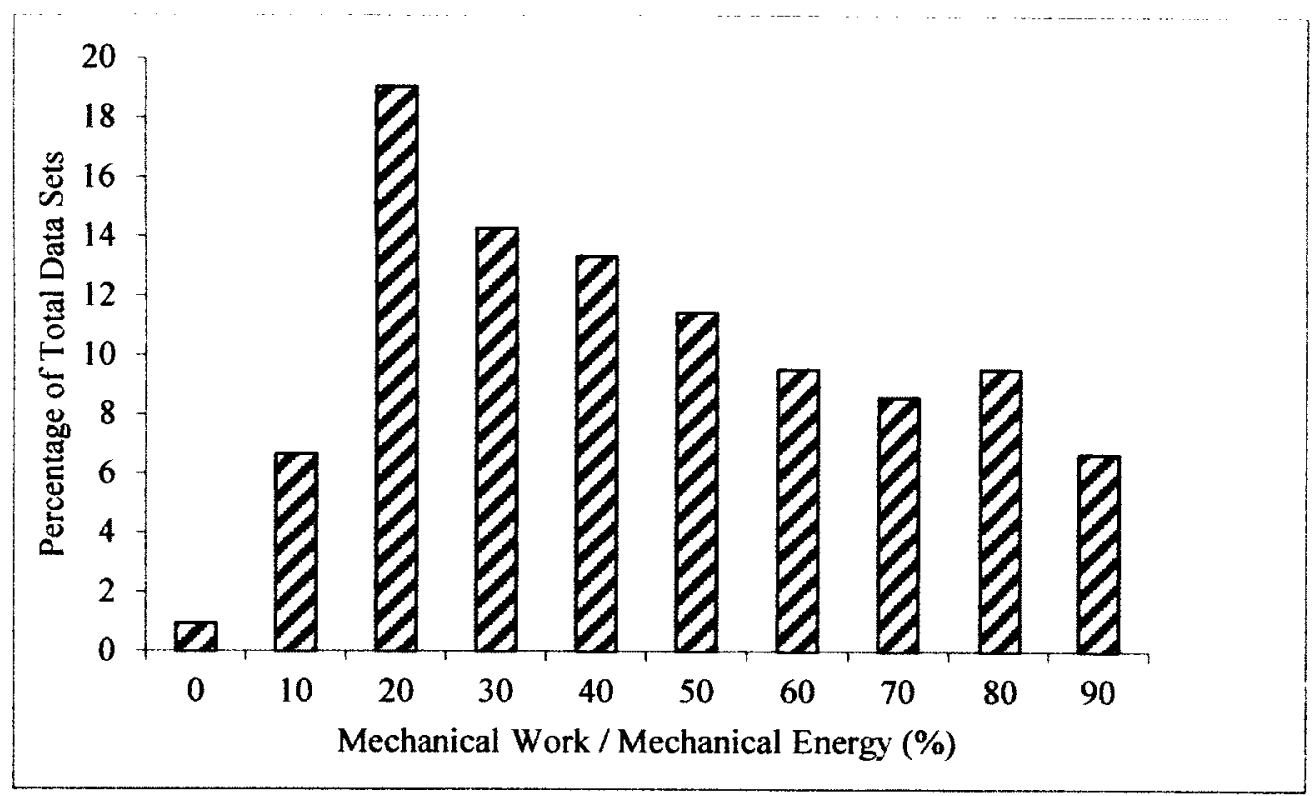

Figure 6.13: Mechanical work/Metabolic energy (\%age) with total data sets (\%age)

Fig. 6.13 shows the data presented in Fig. 6.12 but as a percentage of the total number of data sets. Again it is apparent that 15\%-25\% range of the work/energy ratio is the most frequent.

\subsection{Discussion}

The outcomes that have been observed through data analysis are discussed in the sections below.

\subsubsection{Metabolic Energy with Ship Motion}

A main objective of this research was to examine the effect of ship motion perturbation on the metabolism of humans. Three types of ship motion at four different sea 
states were considered. Metabolic energy data of ten subjects were measured (which included 6 male and 4 female).

The results from Figures 6.3, 6.4, and 6.5 demonstrate that the metabolic energy levels during pitch motions are significantly higher as compared to roll and combined motions. While roll motion demanded higher metabolic energy than the combined motion. The outcomes obtained from our experimental data are similar to the results shown by Wertheim's studies $[1,79]$.

These results lead to the conclusion that during both roll and pitch, greater amounts of muscular efforts are involved for maintaining stabilised posture as compared to combined (or general) ship motion. Muscles are considered as the primary means of stabilizing the body posture by getting feedback from the somatosensory systems of the human body and undergoing contraction or relaxation accordingly. The tension produced in muscles as well as in tendons must be high enough to overcome the gravity effects to maintain the vertical axis of the body aligned with the subjective vertical [81]. In postural stability, the subjective vertical is defined as the position of being in a direction perpendicular to the floor, while the true vertical axis of a subject is defined by the direction of gravity. However, the subjective axis is dependent on various other imputs such as sensory inputs, therefore it may not necessarily be in the exact identical direction as the true vertical axis. In pitch and roll, there are greater opportunities for the body vertical axis to drift from the true subjective axis. This increases the muscle tone (muscular effort) in order to maintain postural stability. This observation holds true physiologically as well, where utilization of more ATP for the increasing muscular tension due to the actin-myosin bond formation translates into more metabolic energy expenditure.

In addition, there is one other important factor that contributes to the need for high energy levels in pitch and roll as compared to combined motion. During roll and pitch motions the visual image of the surroundings perceived by the human visual 
system does not vary as much as the perturbations to which the human body is exposed. This results in a neural mismatch between perceived visual information by the central nervous system and the applied perturbation effects. This neural mismatch is responsible for higher likelihood of body sway which again is characterized by deviation of the true vertical axis away from the subjective axis, recognizing that these two axes need to be aligned for stability maintenance. Hence, extra mechanical work needs to be performed by muscles to align the two axes, and therefore greater metabolic energy levels. During data collection in this work, no canopy or visual movie was provided for the subjects in order to make them feel they are actually exposed to sea perturbations. This limited their visual perception and could be responsible for high neural-mismatch occurences.

Another interesting result is that pitch motion energy levels are higher than roll motion. This result favours the generalized observation about the energy variation with motion type that energy expenditure is more dependent on the pitch motion component in the ship motions [1]. As the pitch factor perturbations lead to more and more postural adjustments as compared to pure roll motion which is predominantly responsible for more MIIs. This could be due to the fact that during a pure pitch motion, the personnel are most likely to step forward or backward in order to maintain balance. Subjects were instructed to be in a standing position rather than walking or doing any activity during data collection which reduces the chances of MIIs in the standing position. Therefore, this state requires less metabolic energy compared to pure pitch motion, where the subject is exerting continuous effort during musculoskeletal adjustments, as this resembles walking up and down a hill which requires continuous involvement of muscles for perturbation adjustment.

The second reason for higher pitch metabolic energy in pitch motion compared to roll motion could be that during our experimental data collection, the subjects were instructed to keep their right foot fixed on the load cell plate. This could be 
responsible for limiting their stance width and hence increasing the musculoskeletal loading to a greater extent due to excursions of the centre of gravity (CoG). Compared to roll motion, with pitch motion there are higher chances for drifting of the centre of gravity (CoG) forward or backward in the pitch motion direction. However, in the roll motion case it is easier to adjust the stance width with the movable left foot.

Another reason for high pitch motion metabolic energy levels is that in pitch motion, the amplitude of the simulated ship deck motion is small but the frequency is very high compared with roll motion which causes subjects to undergo recurrent musculoskeletal adjustments with high frequency, and therefore more and more metabolism is required as muscles are being continuously involved in generating sufficient joint torque by undergoing translations and rotations. While in the case of roll motion, the amplitude of deck motion is higher whereas the frequency is lower. Therefore, muscles are less frequently involved in muscular loading and therefore metabolic levels are expected to be less than with pitch motion effects. These factors are collectively responsible for greater metabolic energy levels associated with pitch motion than roll motion.

The metabolic energy for combined motion increases more linearly between all the motion profiles (i.e., with changing wave height) as compared to roll and pitch motion. For example, by comparing Figure 6.3 with Figures 6.4 and 6.5 , it can be determined that energy levels for the combined/general ship motion follow an increasing pattern where energy consumption increases with each successive motion level (from sea state 1 to sea state 4). For pitch and roll motion, the energy states for sea states 2,3 and 3,4 are similar. This could be because the least severe combined motion state (sea state 1) is comparable to the resting state where the person's metabolic levels are almost equal to resting metabolic levels. As the motion increases in sea state 2, the body starts coming under exposure of increasing motion perturbation effects and therefore energy levels follow a stair pattern by linear increments in metabolism with 
increasing severity until the most severe energy state occurs. Therefore, combined or general ship motion is more linearized in increasing metabolism with each successive sea state as compared to roll and pitch, where the body comes under actual perturbation effects in the least severe sea state therefore increasing the metabolic levels from the resting state. As sea severity increases, the roll motion amplitudes for sea states 3 and 4 become similar, so energy levels are less distinguishable. Pitch motion metabolism findings remain beyond significant explaination for sea states 2 and 3. The major reason for higher metabolic levels in the least severe states could be that sea state 1 has a pitch motion factor of significant amplitude and frequency, which actually raises the metabolism level to the extent that the body comes under the actual effect of perturbation by consuming more and more oxygen for postural maintenance. However, the least severe as well as the most severe energy levels can be easily distinguished for all (pitch, roll, and general) motions by comparing sea state 1 with sea state 4 which shows that metabolism actually increases as the severity increases. In summary, both pure pitch and roll motions demonstrate highly significant metabolic levels compared with the combined motion.

\subsubsection{Gender Factor}

Energy expenditure was also analyzed based on the male-female factor which resulted in interesting results about gender metabolism differences. Males tend to expend higher metabolic energy as compared to females (shown in Fig. 6.7). These energy results for the male-female factor are in agreement with present literature, previously reproduced by Wertheim (1998) [1]. These results also confirm the work of Ferraro et al, who observed that $24 \mathrm{~h}$-energy expenditure outcomes were lower in females than males when body composition, type of activity, and age were adjusted for [76].

The predominant reason for this could be the difference in body mass index (BMI) 
between the two genders. Moreover, another reason could be the fact that the resting metabolic rate (RMR), which is considered as the largest component of metabolic energy expenditure [75], is 23\% greater in males than females. As such, males demonstrate greater RMR and greater metabolic energy expenditure due to the presence of a large quantity of fat free mass. The difference in body composition, which represents the amount of muscle, bone, and fat that make up the human body are considered to be an important factor contributing to differences in metabolic energy expenditure between the genders. Males have more muscle and bone mass and less body fat than females. The muscle mass is considered to be a significant factor for higher ATP consumption. The higher the muscle mass, the higher will be the ATP consumption which could be the reason for higher metabolic rates for men $[75,76]$.

The male-female energy expenditure differences were observed to be similar for pitch and combined motion, while there was a large difference between the two in the case of roll motion. The overall gender difference results are consistent with the metabolism-ship motion variation results as shown in Figure 6.6 which shows that both genders expend the largest amount of metabolic energy in pitch motion as compared to roll and combined motions.

To summarize, lower metabolic energy levels in females as compared to males can be described on the basis of gende-specific differences always present even after making body composition and fat free mass adjustments as suggested by Ferraro et al [76].

\subsubsection{Joint Mechanical Work with Ship Motion}

Human balance is based on the requirement of maintaining the centre of mass (CoM) within the base of support $(\mathrm{BoS})$. Different joints contribute in order to maintain the stabilised human posture during perturbations.

Posture maintenance is based on the muscle contraction phenomenon which takes 
place and is associated with different joints in the body. Among the various joints, the ankle, knee, hip, and head-neck joints are considered the predominant joints for postural stability. Generally, calf muscles (lower leg muscles) are the initiallyactivated muscles whenever the human body is subjected to motion perturbation. Calf musculature activation is followed by soleus, hamstring, and neck muscles including supraspinalis muscles. However, upper extremity joints (i.e., shoulder, elbow, and wrist) contribute to a much lesser extent to maintain postural stability. The plots of Section 6.4 confirm these observations, where ankle joints play a dominant role in maintaining postural stability. This is followed by the contribution of the headneck joint as well as hip and knee joint activation while other upper extremities play insignificant roles in maintaining postural stability [74].

In general, three important factors contribute to stability maintenance:

- Stabilizing the human body and head against gravity;

- Maintaining the centre of mass (CoM) within the base of support (BoS) by adjusting stance width; and

- Voluntary body movement involved with anticipation of goal-oriented responses.

Balanced position is achieved through the integrated movement of all body segments in a coordinated pattern so that the body is subjected to minimal sway. Physiologically, the tibialis anterior muscles (in front of the leg near the lower shin in calf musculature) are predominant muscles for stability maintenance as they control the foot stability which in a way adjusts the stance width and therefore controls overall body posture [81]. These muscles are mainly responsible for involving all lower extremity joints (i.e., ankle, knee, and hip) to perform mechanical work. Calf musculature activation is accompanied by co-activation of the head-neck joint as well as hamstring muscles for keeping the human body movement within limits. This sequence is accompanied by feedback obtained from the neuromuscular structure of the 
human body which mainly focusses on the head and lower extremities (i.e., lower leg involving ankle and knee joints) for stable posture. Three sensory mechanisms (i.e., vestibular, visual, and somatosensory systems) are integrated together in order to provide feedback regarding changing position and maintaining it to be stabilized as much as possible. The vestibular system controls the head orientation deviation from the gravitational axis, while the visual system detects head movements due to visual feedback obtained through eye reflex actions. In other words, this control action can be performed through two different functions: static and dynamic. The dynamic function controls the reflex action of the eye by tracking head movements in space through a vestibulo-ocular reflex action while the static function allows head position monitoring through the vestibulo-spinal reflex action. The existing literature indicates that head-neck muscle fatigue (which limits possibilities for head translation and rotation) results in greater postural sway due to cervical dizziness [78]. Moreover, vertigo arising from vestibular dysfunction also indicates that head-neck muscles play an important role in controlling postural sway with head movements [78]. This increased postural sway during head-neck muscle fatigue could be due to erroneous sensory input provided to the central nervous system about the postural state. It results in greater risk of falling. Furthermore, visual information is considered as another important factor for postural stability mechanisms. Recent studies show that body sway risks increased from $22 \%$ to $56 \%$ for an eyes-closed case as compared to an eyes-open case [78]. Head stabilization during stability maintenance is considered as significant motor control which provides an orientation-stabilized platform to the human body for smooth progression of body movements [78]. This is done to resist any sway and it is heavily based on the input provided by different sensory systems (i.e., vestibular and visual systems for this task). The third important sensory system, (i.e., proprioception system) observes leg orientation with respect to the support surface. The lower extremity joints are also considered to be dominantly involved in 
postural stability by implementing ankle and hip strategies which are discussed next. Ankle and hip strategy for joint mechanical work

The ankle strategy is characterized by modelling body sway as a single inverted pendulum which allows the body to keep feet attached to the floor of the platform. The hip strategy is characterized by modelling body sway as a double inverted pendulum where segments are articulated at the hip and are most likely active during fast translation on the motion platform.

Ankle joints are considered as dominant joints, which are responsible for maintenance of stabilized posture, compared with all other joints. Ankle motion is more likely to limit CoM position within the base of support. Thus, they are considered a dominant factor for balance control. This could be because the ankle joint is located at the position farthest from the centre of mass of the human body when compared to other lower extremity joints. This location facilitates activation of dorsal ankle muscles followed by activation of dorsal thigh and trunk muscles.

As motion disturbance becomes more rapid, the hip joint becomes equally involved in maintaining stability, until both dominant joints contribute mutually to bring the human body back to the recovery phase. In using a mixed strategy, both flexion at the hip and extension at the ankle joint are activated for balance maintenance. The mixed strategy works on the basis of activation of ventral thigh, hip, and knee muscles which are followed by activation of dorsal muscles. Therefore, the hip strategy is based on defining body sway as a double segmented inverted pendulum which is partitioned at the hip. In other words, this strategy represents body sway as a double inverted pendulum by generating counter-phase torque at both hip and ankle joints [74]. These strategies are based on a muscle activation phenomenon which identifies the predominant muscles that activate first in order to respond to the changing body sway. Kuo's model [73] shed light on both these strategies by explaining that humans are more likely to drift to the hip strategy from the ankle 
strategy as the platform motion becomes faster as well as more severe (i.e., larger amplitude and frequency). This strategy also involves ankle and knee joints equally. By continuously touching the ground while keeping the knees straight, the knee is considered to be performing mechanical work by producing muscle tension for this position. Keeping the knee in a straight position also requires muscles to be active in order to provide some constraint for this strategy. These observations indicate that during less severe motion, the ankle muscles are predominantly involved in performing mechanical work to maintain postural stability. As the severity of motion increases, hip muscles start contributing significantly while still keeping ankle and knee muscles active for generation of muscular tension. That is why the latter strategy is known as a mixed strategy rather than a pure hip strategy. This conclusion confirms the Nashner and McCollum (1985) results that rotation takes place primarily through the ankle joint rather than being exclusively through the ankle joint [74].

Joint mechanical work is the result of muscle activation at a particular joint that is involved in maintaining a stable position. In terms of physiological phenomena, it can be observed that the greater the number of muscle fibres involved, the greater the muscle force production due to fibre contraction and also the greater is the mechanical work done by the muscles for a particular change in joint angle. Therefore, calculation of joint torque and the associated mechanical work provides a measure and understanding of muscle activation patterns related to different body segments as well as control strategies (ankle, hip, or mixed) for various deck motion severities.

The results of this investigation confirm the ankle and hip strategy, such that for less severe motion states, the ankle joints are activated first. These are supported by increasing knee and hip joint activation as the motion severity increases. At the same time, the data indicates that the head-neck joint always produces significant mechanical work during ship motion, but its relative contribution decreases as the severity increases. This could be explained by the fact that during less severe ship 
motion, the CoG is maintained mainly by the ankle and head-neck joints, which are located at the extreme distal and proximal ends of the subjective vertical axis and are most likely to undergo translation and rotation. As the motion severity increases, this task is distributed amongst other body joints. As described for joint mechanical work results (Figures 6.8,6.9, and 6.10), the hip and knee mechanical work contributions remain present in low ship motion states indicating that knee and hip muscles are still active in less severe sea states but contributing minimally to stability. In conclusion, the existence of the mixed strategy for less severe ship motion cannot be completely ignored although the ankles are the major contributor to stability and balance.

Moreover, during less severe sea states (Figures 6.8, 6.9, and 6.10a and $b$ ), upper extremity joints (i.e., shoulder, elbow, and wrist joints) contribute insignificantly to postural stability. It should be pointed out that the subjects were instructed to try to remain in a standing still position focussing mainly on lower extremity joints (mainly ankle, knee, hip, and L5-S1) and head movements which co-activate head-neck muscles simultaneously. Similarly, L5-S1 joint (lumbar-sacral joint) mechanical work increases progressively as the motion severity increases. Furthermore, during pitch motion for sea state 3 (Fig. $6.10 \mathrm{c}$ ), the wrist joint is very active as during this motion state, participants were more likely to grab the railings of the motion platform in order to avoid falling which contributed to wrist joint involvement. Finally, the mechanical work contribution by the wrist and L5-S1 muscles point to the fact that some muscle patterns that do not undergo co-contraction in less severe motions, eventually become active as the motion amplitude and frequency increase. This explains the reason for distributing the mechanical work from ankle joints to all other body joints as the motion becomes more severe.

\section{Joint Work Contribution Comparative Analysis for Different Sea Motions}

The total mechanical work contributed by different body joints varies with the specific ship motion. For instance, during pitch motion, a person exerts more effort 
to maintain his stability as compared to roll and combined motions. Therefore, the mechanical work done by all joints during pitch motion is more significant as compared to roll motion where the lower extremities (i.e., ankle, knee, and hip) and head joint are primarily involved in stability maintenance during all sea states. For combined motion, when the sea state becomes severe up to the maximum level (i.e., 7 meter waves), the mechanical work gets equally distributed amongst all the joints. This suggests that the involvement of upper extremity joints becomes more prominent with the increase in the severity of the sea states during combined motion.

Through analysis of the metabolic levels for these three motions, it is observed that more involvement of joints results in higher metabolism. Since the work done during pitch motion is a contribution from all the body joints, higher metabolic outcome is observed for this type of motion. Likewise, the stability is maintained in the roll motion mainly through lower extremities which results in lower metabolism for roll as compared to pitch motion. The metabolic outcome for combined motion is the least; however, the results show an increase in metabolism when the severity of the sea state increases as the upper body joints come into play to perform the required work.

\subsubsection{Vertical Ground Reaction Force Component Variation with Ship Motion}

As pointed out previously in Section 6.8 .3 , the subjects were prone to make recurring adjustments to their posture. This ultimately increases the muscular work and is responsible for increased metabolic energy expenditure with the ship motion severity. These results agree with the existing literature [71]. Hoang et al reported that mildly and severely crouched posture (which is supposed to be obtained during severe ship states in the present study) is associated with high joint muscular tension which in 
turn increases the force which is equally and oppositely applied by the floor to the foot in order to maintain stability [71]. This was associated with high metabolic energy levels. Moreover, these observations are similar to the results presented by Robert

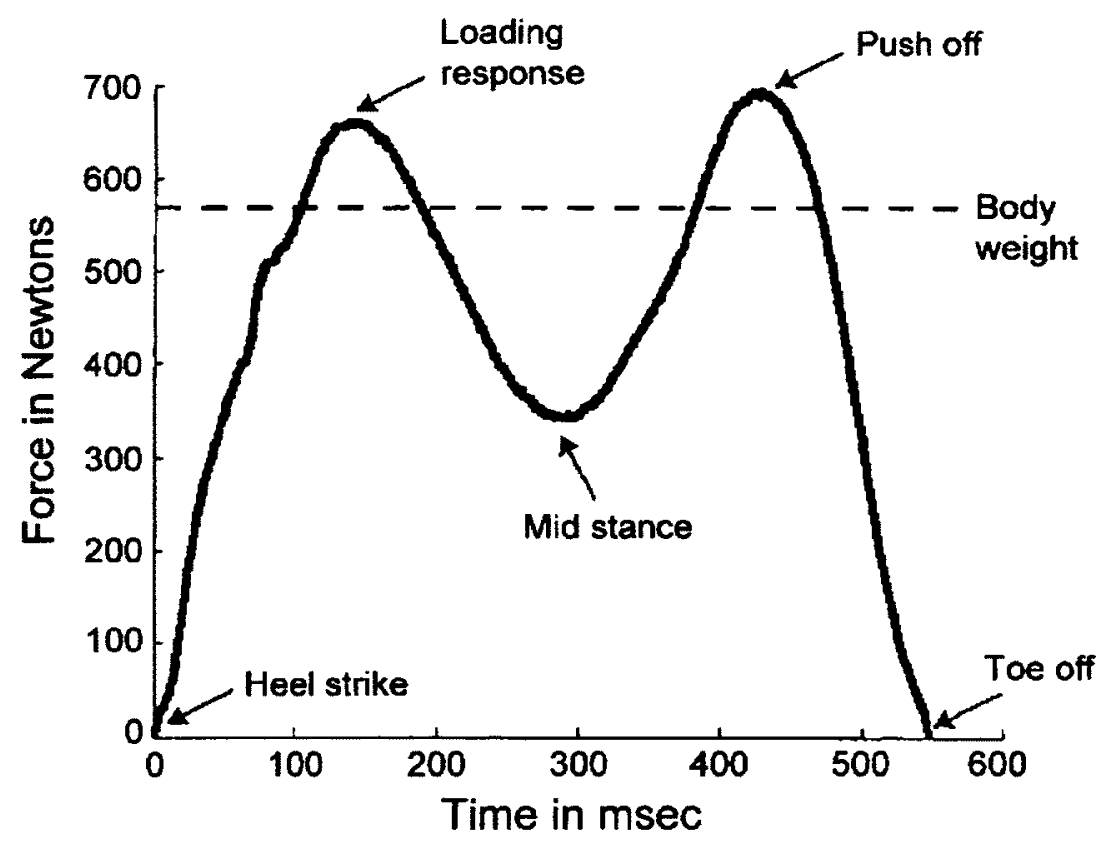

Figure 6.14: Vertical component of GRF characterized by double-hump curve during walking [70].

et al [70], which concluded that during walking, stress applied to the feet is much greater as compared to standing still conditions. This reflects that a larger force is required to maintain a stabilised posture when humans try to maintain posture by recurringly changing their stance width by taking steps forward or backward. As shown in the results presented by Robertson et al, the vertical peak component of the ground force during walking is 1.2 times the body weight as compared to standing still where the vertical peak component is equally distributed between two feet to balance the body weight [70]. This could be because every time a person takes a step or makes adjustments with body segments in order to maintain stability, the gait stance pattern is being followed where different parts of the feet touch the floor 
during different stance phases.

Therefore, the vertical component of the GRF for gait patterns can be demonstrated by the double hump curve, which characterizes the heel strike and toe push-off steps in this pattern (refer to Fig. 6.14) [70]. The vertical GRF component is expected to increase with muscle tension produced during severe ship motion conditions. This is because the surface area of plantar support varies with deck motion severity which most likely causes stepping and changing stance width to stabilise posture [70]. These

factors are collectively responsible for the increased vertical GRF components during rapid motion states.

\subsubsection{Mechanical Work-Metabolic Energy Correlation}

The correlation results shown in Table 6.1 indicate that there is significant correlation between the metabolic energy expenditure and mechanical work of the subjects. These results agree with Hill's results in muscle model studies (i.e., Hill 1938, Woledge 1985) $[64,66]$. In these studies, they used a muscle model to show that for isolated muscles, there exists a direct relationship between mechanical work done by muscle and the metabolic energy expended by it due to utilization of ATP while undergoing contraction due to actin-myosin bonding. In other words, the increase of the mechanical work done by the body correlates to an increase of $\mathrm{O}_{2}$ consumption which raises the energy levels due to more and more ATP utilization.

Hill's muscle model conclusion has been a main consideration in the investigation of postural stability. To date, a few studies (Burdett et al, 1983; Cavagna and Kaneko, 1977; and Willems et al, 1995) suggest numerous methods to calculate mechanical work performed by the body and limbs $[83,84]$. However these studies have not been able to provide the reason behind why and where the metabolic energy is compensated for while walking or performing normal gait activities. Moreover, recent inverted pendulum model experiments conducted by McGeer, Kuo, and Garcia et al, during 
walking trials (McGeer, 1990; Alexander, 1995; Garcia et al, 1998; and Kuo, 2002) concluded that mechanical work is not necessarily performed between each and every successive step, but between the steps [85]. For example, mechanical work is an important factor of metabolic cost while walking if a biped model is used. In this case, an inverted pendulum is used to model a single support base; and a stance limb defines the centre of mass moving along an arc. The inverted pendulum model requires no mechanical work to be performed in order to move along the arc, but there is some mechanical work done when there is a change from one stance limb to the other stance limb. Moreover, the mechanical work done while changing stance limb can be categorized as either positive work or negative work. Positive work occurs whenever restoration of energy loss takes place, while negative work occurs for redirecting the centre of mass velocity from one arc length to the other. These studies further explained that there will be exactly the same metabolic energy expended for mechanical work done if muscle efficiency is considered as constant for each successive step transition [85].

However, in the present study, there is the requirement of maintaining postural stability during severe ship motion. Hence the mechanical work performed by different body joints could not necessarily follow the exact linear expenditure of metabolic energy for that stability task. Muscle efficiency is no longer constant in nature. This is because with motion severity there will be greater repetitive postural adjustments made for ensuring stability. This results in more frequent musculoskeletal loading and lesser linearity in muscle efficiency (amount of metabolic energy absorbed per unit number of muscle fibres activated) due to haphazard muscle contraction. Therefore, some randomness in the mechanical work-metabolic energy correlation curve is possible which is responsible for deviation of p-values from exactly 1 , which represents $100 \%$ efficiency in muscle fibre contraction to metabolic energy expended. Another important reason behind this observation could be that mechanical work performed by 
joints is being calculated through mathematical modelling of the human body (i.e., inverse dynamics) rather than implementing any invasive technique to detect how many and which particular muscle fibres are contracting by ATP utilization. Also, motion capture data were edited manually due to a number of data gaps caused by railings across the platform. It could be considered as a reason for lower motion data accuracy. Another observation that can be made from these results is that due to a lack of efficiency in the conversion of biochemical energy into mechanical energy during actin-myosin cross bridge formation, there are possibilities of utilizing metabolic energy in other physiological processes such as sarcoplasmic reticulum $\mathrm{Ca}^{2+}$ pumping (which is a prerequisite for cross bridge formation) [66]. Also, it is not necessary that all biochemical energy (i.e., ATP) be used up for mechanical work performed by muscles, as there is energy used up in other activities as well, such as thinking and other mental processes which are not necessarily categorized under mechanical work performed by the muscles.

\subsubsection{Direct Comparison of Mechanical Work with Metabolic Energy}

Another significant outcome which was expected from this research was a quantitative estimate of the extent to which mechanical work done by the human body accounts for the metabolism levels of the human. In total 105 data sets were analyzed corresponding to the 10 research subjects subjected to each of the four levels of deck motion severity. The distribution of the ratio of mechanical work performed to total metabolic energy was plotted (Figures 6.12 and 6.13 ) both in terms of raw numbers of instances and as a percentage of the total number of cases. The results show that while the ratio is quite variable, there is a distinctly noticeable elevation in the rate of instances where the ratio falls in the $15 \%-25 \%$ range. This indicates that for the 
motion cases considered, the subjects most often expended approximately $20 \%$ of their total metabolic energy actuating muscles associated with maintaining postural stability. Further, in $67 \%$ of the cases studied the ratio of mechanical work to total metabolic energy is below 50\%. In addition, another important and expected observation is that the ratio can never reach $100 \%$ because some of the subjects metabolic energy must be associated with processes within the human body other than physiological effort. While evident, consistency between this expected result and the result of the fairly complex sequence of computations involved in this research is considered to be a favourable outcome.

\subsection{Limitations}

It should be noted that the results obtained in this investigation are subject to the following limitations:

1. The age of the 10 selected subjects was in the range of $20-28$ years during experimental trials for this research. In a future work, a widening of the age range of the subjects should be considered.

2. The experimental trials were performed within the labratory, which limited the sea roughness exposure to the human subjects. This might have an impact on the postural outcomes compared to experimental trials performed at sea.

3. For ship motion simulation, the motion platform simulated rotations in 3 degrees for different sea states. Translations were not considered for the motion profiles due to motion limits of the ship motion simulator. 


\section{Chapter 7}

\section{Contributions, Conclusion, and Future Work}

\subsection{List of Contributions}

There are 6 major contributions which have been discussed for the thesis (Chapter 6):

1. 3-Dimensional multibody dynamic model development;

2. Metabolic energy variations with different sea states for pitch, roll, and combined motion;

3. Evaluation of metabolic energy variations on a gender basis;

4. Calculation of joint mechanical work distribution for 3 motion types;

5. Mechanical work-metabolic energy relationships with sea roughness;

6. Impact of ship motion on vertical ground reaction force. 


\subsection{Conclusion}

The maintenance of postural stability of shipboard personnel while at sea is a major concern in the maritime sector. Comprehensive knowledge of motion-induced fatigue as well as disorders, and work and energy expenditure by humans in different motion environments is required to maximize the crew performance and motivation, and to devise safety standards to provide a better and safer workplace for all shipboard persomnel. This was the main motivation behind the current research where mechanical work performed by the body, the work distribution between different joints, and the rate of metabolism required to maintain postural stability for different sea states were investigated. To this end, a three-dimensional multibody dynamic model of the human body was developed and validated in MATLAB. The model was used to calculate the work done by different joints and the body to maintain postural stability. Experimental data were collected from 10 participants for 12 different simulated ship deck motion profiles (i.e., roll, pitch, and combined motion for four different sea conditions). The data were obtained using a load cell, a motion capture system, and a metabolic analyzer. Simultaneously, metabolic energy of the subjects for all sea states was determined to examine the metabolic activity of the subject with varying sea states.

The results show that an increase in sea motion results in more energy expenditure to maintain stable posture. A high correlation between mechanical work and metabolic energy for all levels of sea state was observed. A multibody dynamic model was used to determine the mechanical work done by different body joints. The results suggest that the contribution of various joints to the total mechanical work of the body varies for different sea state profiles. For less severe pitch, roll, and combined motion, the lower body joints are the primary contributors to the work. As the sea state becomes more severe, the upper extremity joints become more involved. The 
upper body joints are more prominent contributors in severe pitch and combined motions whereas for severe roll motion, the mechanical work continues to occur within the lower extremities.

To summarize, the results suggest that the deck motion conditions have a significant impact on metabolism, mechanical work done, and joint workload of personnel during representative shipboard tasks. It is therefore recommended that human energy expenditure requirements be considered in the planning of shipboard activities, particularly in elevated sea conditions, and that they ultimately also be considered in ship design.

\subsection{Future Work}

This research provides significant findings about mechanical work performed as well as metabolic energy expended by personnel in order to maintain their postural stability in various sea states. During this investigation, the subjects were standing while trying to maintain their stability. Extension of this research should consider the postural stability of the subjects during various simple tasks such as manual material handling, loading, and lifting conditions. This investigation would determine task-specific metabolic energy correlations with mechanical work performed. This will better depict how MIF effects are associated with different tasks handled by the crew in motion-rich environments. The results of that investigation can contribute to a better understanding and successful planning of ship deck tasks by suggesting task-specific work shift adjustments as well as nutrition requirements. This will lead to avoiding performance degradation occurences by minimizing biodynamic impediments.

Moreover, development of a robust simulation model for postural stability analysis by using graphics-based tools available in software such as SimMechanics may 
be considered. SimMechanics is a simulation environment within MATLAB, where modelling of multibody dynamic system takes place by using different blocks for representation of bodies, joints, constraints, and force elements. A SimMechanics implementation could be considered as an alternative way to represent multibody dynamic systems. This approach could be used as a validation tool and for exploring further details of the multibody dynamic model by providing real-time visualization of dynamics during the simulation. 


\section{Appendix A}

\section{Sample Results for a Single Subject}

This appendix contains a detailed description of the analysis of data obtained from the subjects that participated in the research. In the interest of brevity, the results of data collected from a single participant are given as a sample. The procedure used in investigating the data for all 10 subjects is consistent with the one mentioned here.

\section{A.1 Metabolic Energy Variation with Simulated Ship Motion}




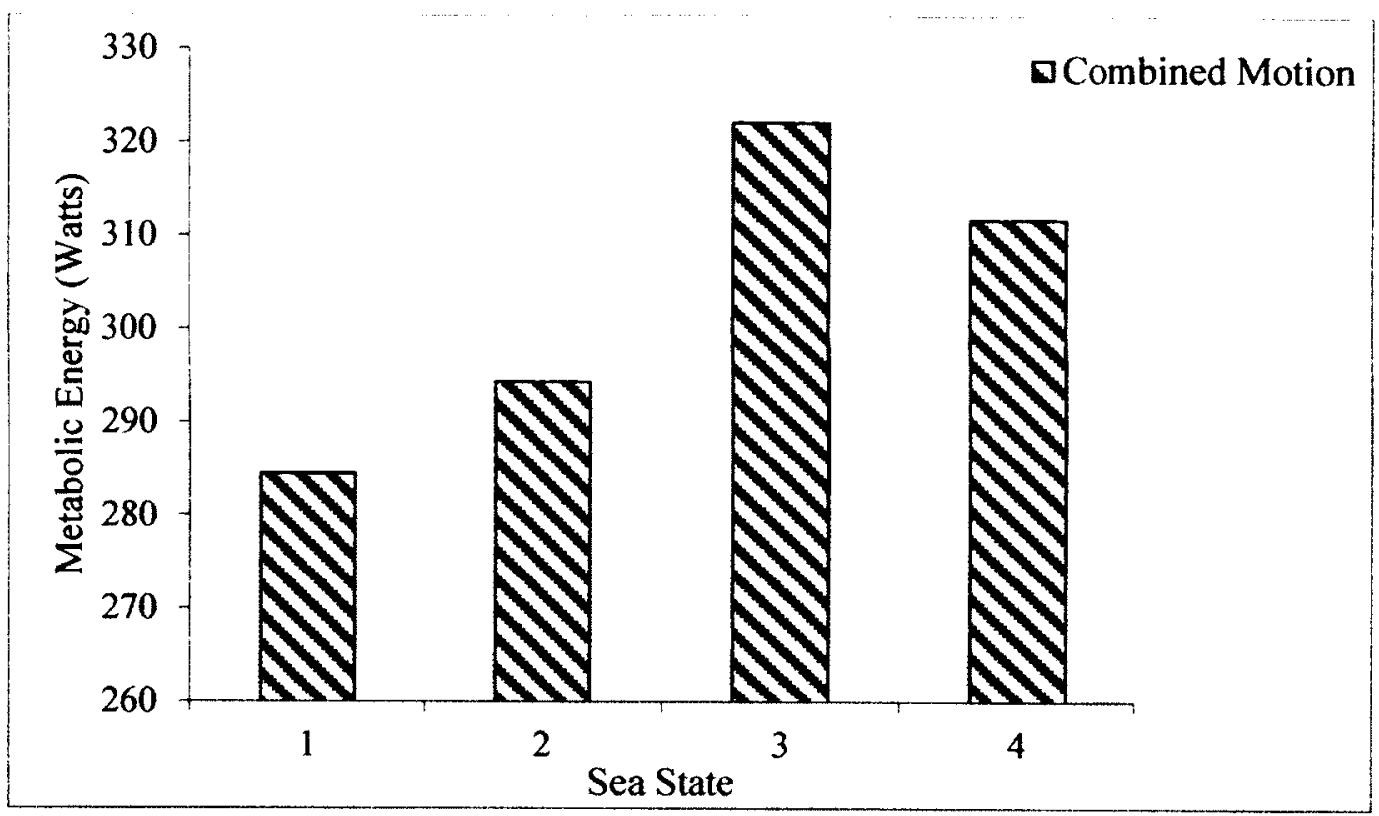

Figure A.1: Energy variation with combined motion for subject no. 4

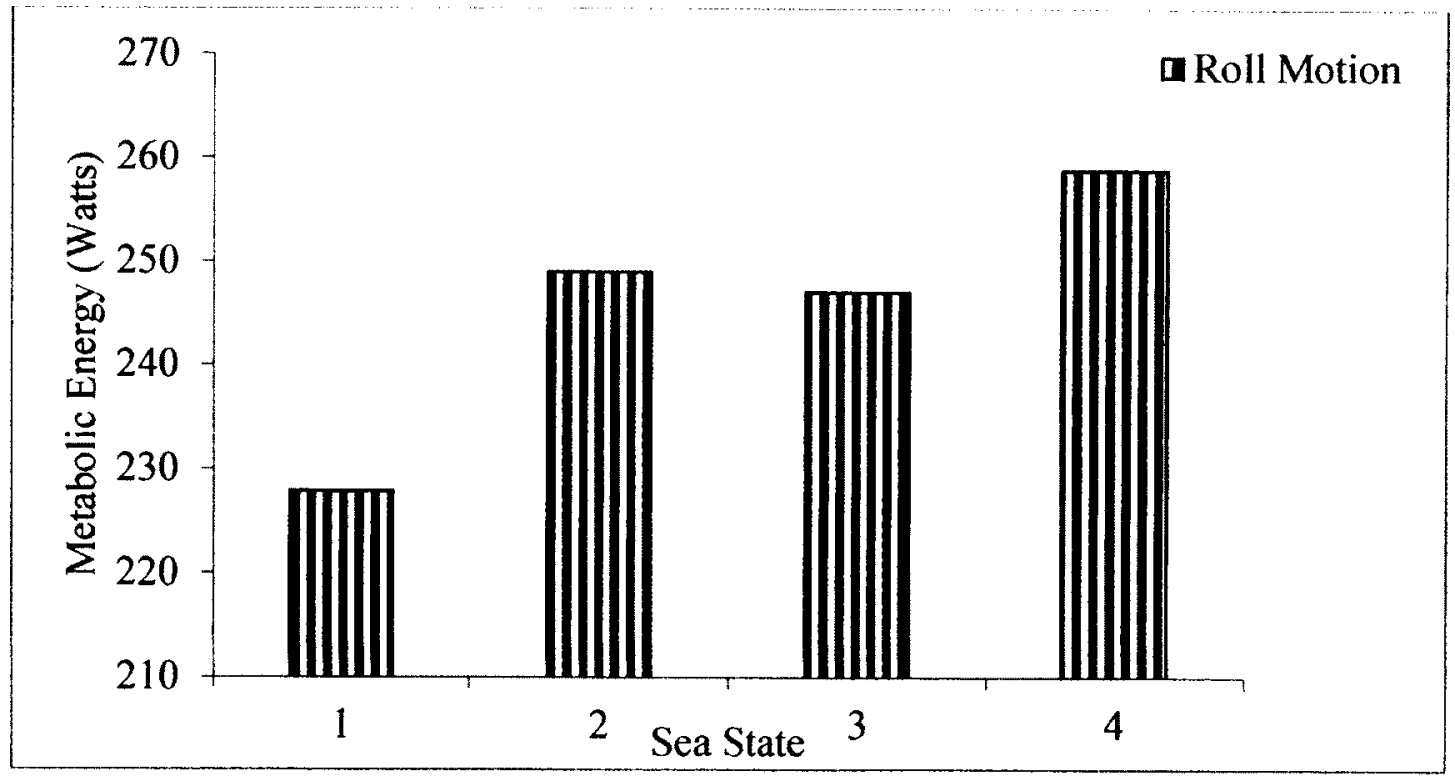

Figure A.2: Energy variation with roll motion for subject no. 4 


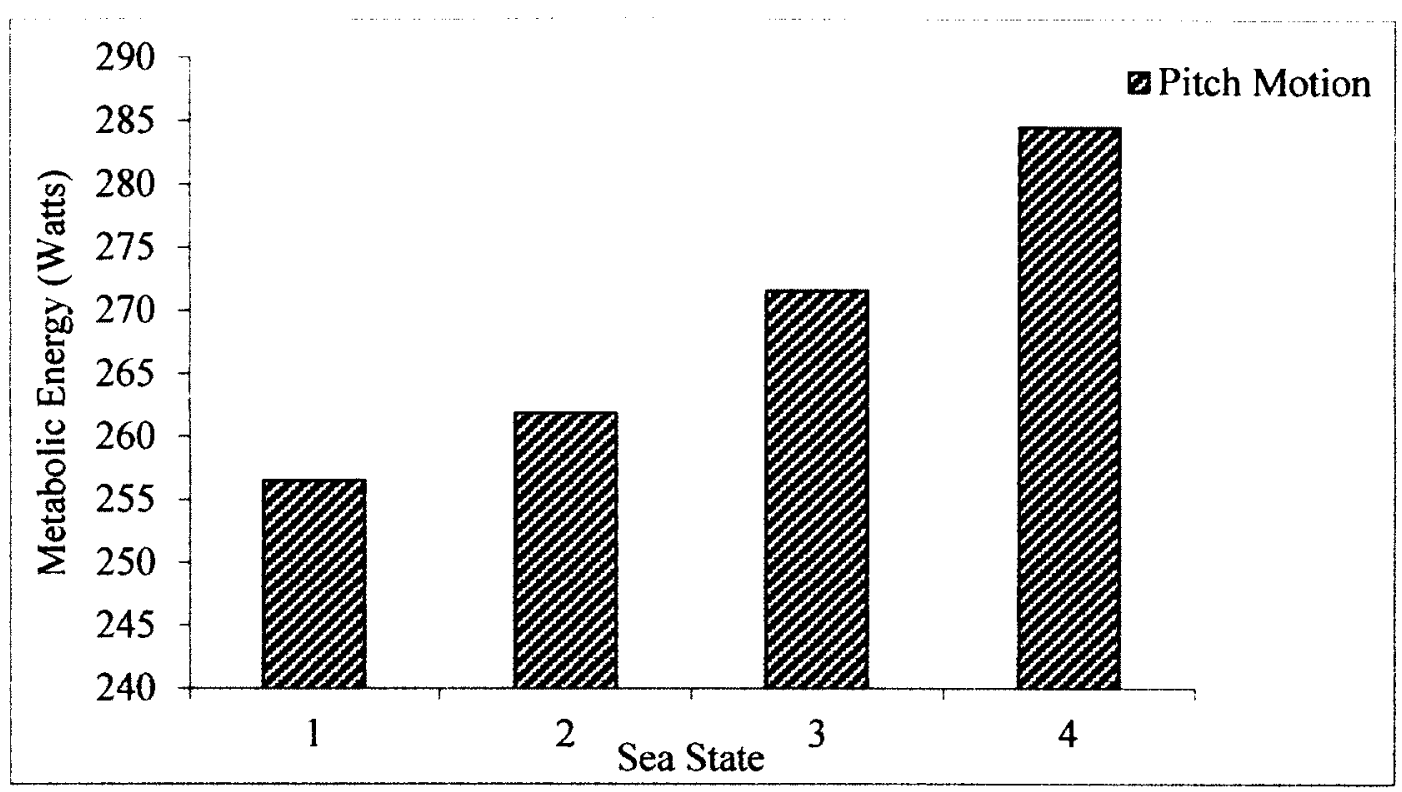

Figure A.3: Energy variation with pitch motion for subject no. 4 


\section{A.2 Joint Work Contribution For Different Ship Motions}

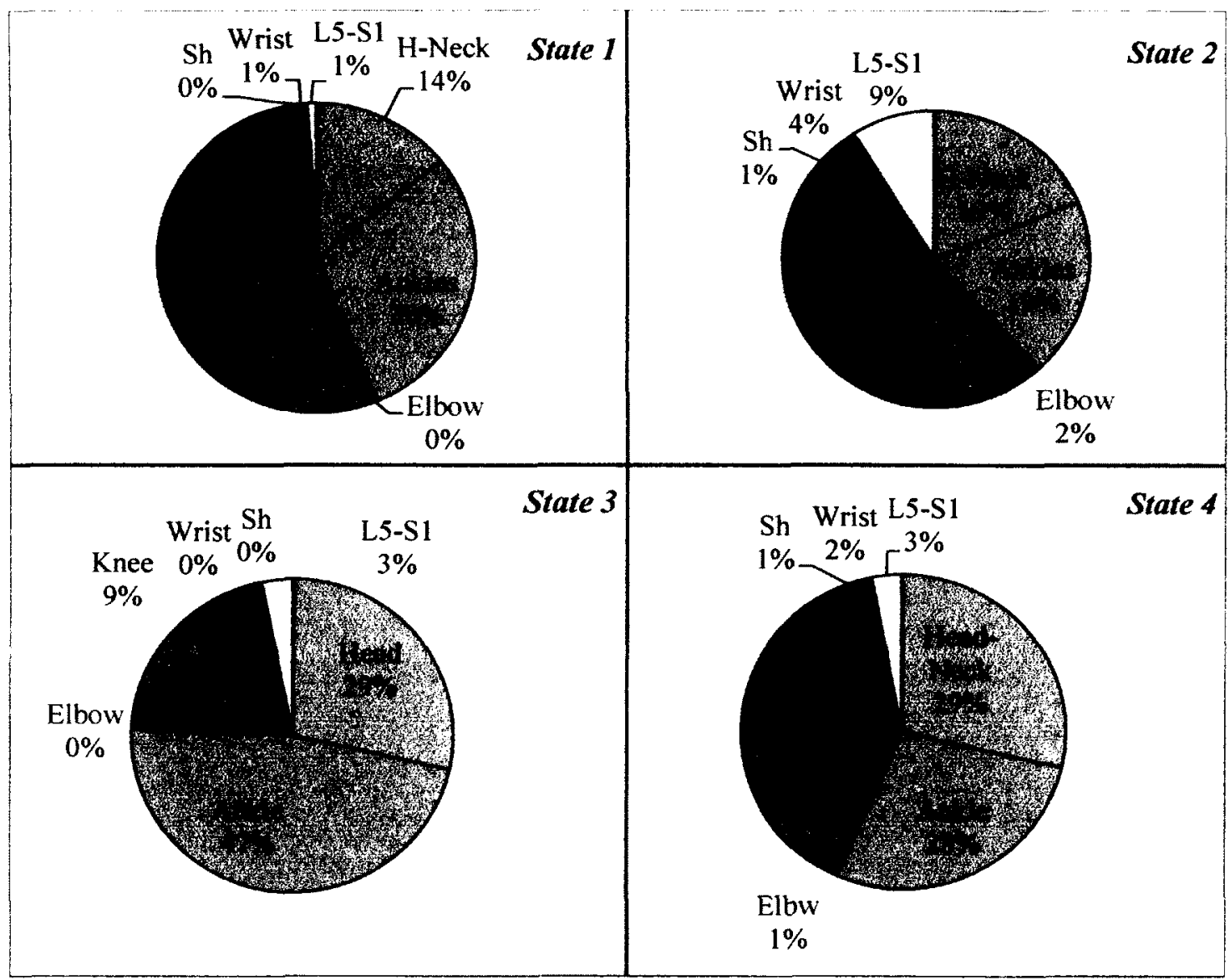

Figure A.4: Joint work contribution for combined motion for subject no. 4: (a) 1 meter waves (least severe); (b) 2 meter waves (medium); (c) 5 meter waves (severe); (d) 7 meter waves (most severe) 


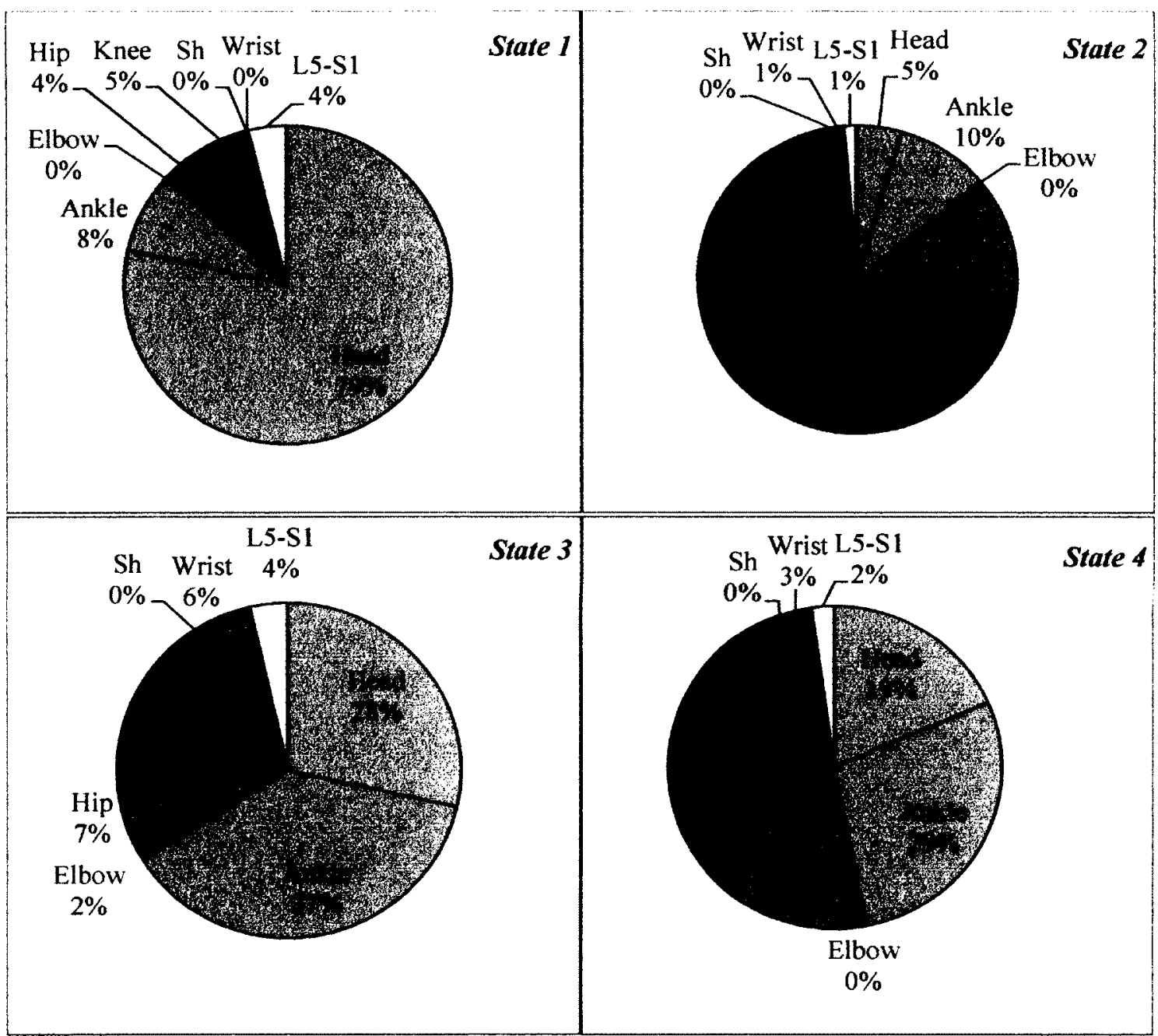

Figure A.5: Joint work contribution for roll motion for subject no. 4: (a) 1 meter waves (least severe); (b) 2 meter waves (medium); (c) 5 meter waves (severe); (d) 7 meter waves (most severe) 


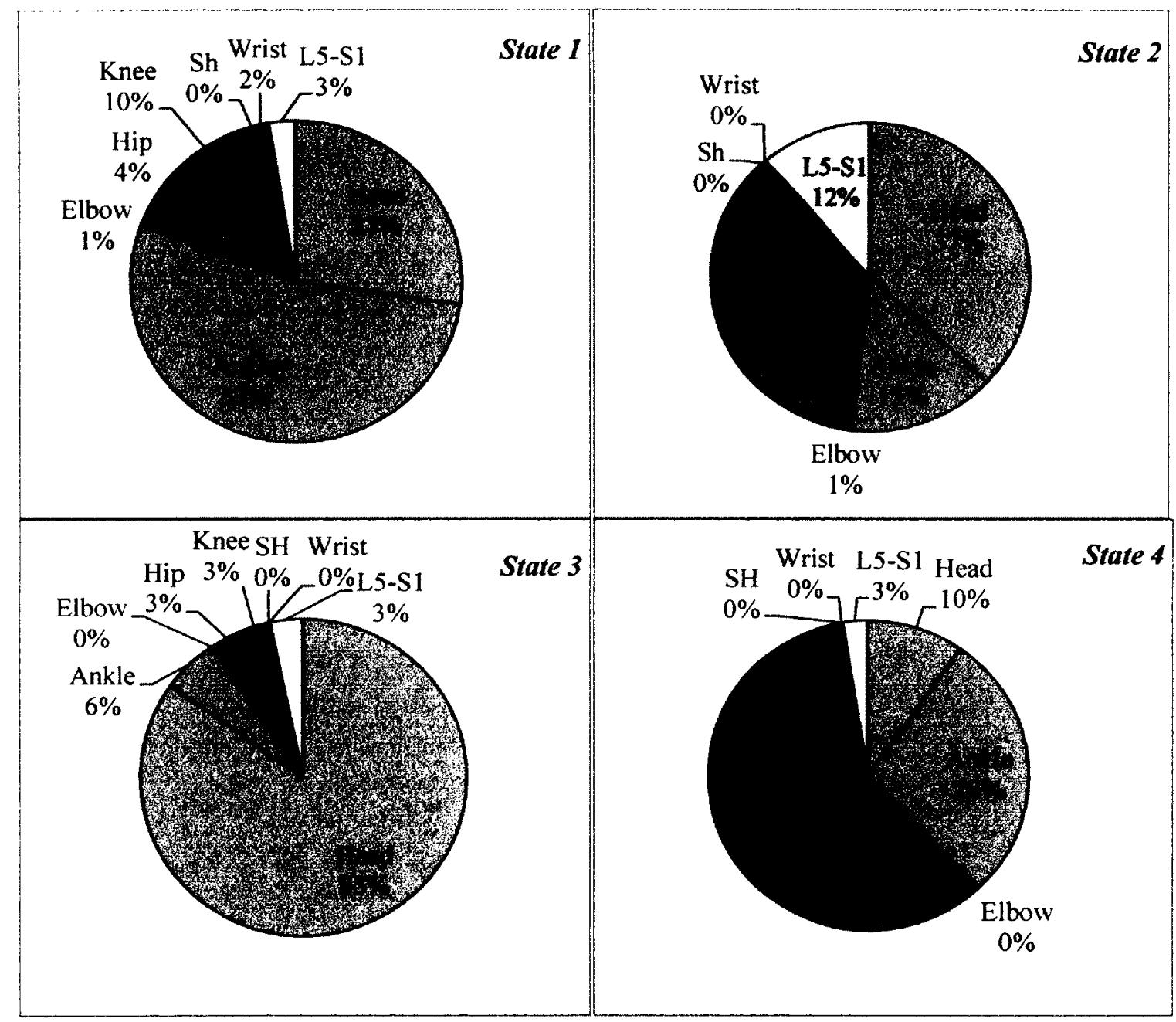

Figure A.6: Joint work contribution for pitch motion for subject no. 4: (a) 1 meter waves (least severe); (b) 2 meter waves (medium); (c) 5 meter waves (severe); (d) 7 meter waves (most severe) 


\section{List of References}

[1] Wertheim A.H., Havenith G., Heus. R., "Human energy expenditure when walking on the moving platform," Journal of Applied Physiology,Vol.77, pp. 388-394, 1998.

[2] Riola J.M., Garcia de Arboleya M., "Habitability and personal space in seakeeping behaviour," Journal of Maritime Research,Vol.3, No.1, pp. 41-54, 2006.

[3] Dobie T.G, "The importance of the human element in ship design," Presented at Ship Structure Symposium, Arlington VA,June 2000.

[4] Dobie T.G., "Critical significance of human factors in ship design," Proceedings of RVOC Meeting, pp. 1-23, 2003.

[5] Marais J.F., Basset F.A., Duncan C.A., Mackinnon S.N., International Conference on Human Performance at Sea (HPAS), pp. 1-7, 2010.

[6] Crossland P., Rich K, "Validating a model of the effects of ship motion on postural stability," International Conference of Environmental Ergonomics, Vol.77, pp. 385-388, 1998.

[7] Stevens S.C., Parsons M.G., "Effects of motion at sea on crew performance: A Survey" Marine Technology,Vol.39, No.1, pp.29-47,January 2002.

[8] Peeters H.P.,Caberg H.B., Mol. J.M.F., "Evaluation of biomedical models in posturography," Medical and Biological Engineering and Computing, Vol.23, No. 5, pp. 469-473, 1985.

[9] Benvenuti F., "Physiology of human balance," Advanced Neurology,Vol.87, pp.41-51, 2001.

[10] Nashner L.M., "Adaptation of human movement to altered environments," Trends in Neuroscience, Vol.5, pp. 358-361, 1982. 
[11] Winter,D.A., Patla A.E., Prince F., Ishac M., Gielo-Perczak K., "Stiffness Control of balance in quiet standing," Journal of Neurophysiology, Vol.80, pp.1211$1221,1998$.

[12] De Luca,C.J.,LeFever R.S., McCue M.P., Xenakis A.P., "Control scheme governing concurrently active human motor units during voluntary contractions," Journal of Neurophysiology, Vol 329, pp.129-142,1982.

[13] Bothner K.,Jensen J., "How do non-muscular torques contribute to kinetics of postural recovery following a support surface translation," Journal of Biomechanics, Vol.34, No.2, pp.245-250, 2001.

[14] Koozekanani S.H.,Stockwell C.W., McGhee R.B., Firoozmand F., "On the role of dynamic models in quantitative Posturography," IEEE Transactions on Biomedical Engineeroing,Vol. BME-27(10), pp.605-609, 1980.

[15] Lewis C., Griffin M.J., "Evaluating effects of deck motion on postural stability," ISVR Contract Report No.95 by 12,University of Southampton, 1995.

[16] McGhee R.B., Kuhner M.B., "On the dynamics stability of legged locomotion systems," Technical Report, University of Southern California, 1970.

[17] Nashner L.M., "Sensory feedback in human posture control," Massachusetts Institute of Technology, Ph.D. Dissertation, 1970.

[18] Hemami H., "The inverted pendulum and biped stability," Mathematical Biosciences, Vol.34, No.2, pp.95-110, 1977.

[19] Geurssen J.B., Altena D., Massen C.H., Verduin M., "A model of the standing man for the description of his dynamic behavior," Agressologie, Vol.17B, pp.6369,1976 .

[20] Hemami H.,Jaswa V.C., "On the three-link model of the dynamics of standing up and sitting down," IEEE Transactions on Systems, Man, and Cybernetics, Vol. SMC-8, pp.135-120,1978.

[21] Stockwell C.W., Koozekanani S.H., Barin K., "A physical model of human postural Dynamics," Annals of NewYork Academy of Sciences,Vol.374, pp: 722730,1981 .

[22] Nawayseh N.,Griffin M.J., "Effect of frequency,magnitude and direction of translational and rotational oscillation on postural stability of standing people," Journal of Sound and Vibration, Vol. 298, pp:725-754,2006. 
[23] Kodde L.,Caberg H.B., Moi J.M.F., Massen C.H., "An application of mathematical models in posturography," Journal of Biomedical Engineering, Vol. 4, pp.44-48,1982.

[24] Riley P.O.,Mann, R.W., Hodge,W.A., "Modelling the biomechanics of posture and balance," Journal of Biomechanics,Vol.23(5), pp.503-506,1990.

[25] Patton J.L., Pai Y.-C., Lee W.A., "A simple model of the feasible limits to Postural stability," 19th International IEEE Conference, Chicago,IL. USA.,Vol. 4, pp. 1679-1682,1997.

[26] Humphrey L., Hemami H., Barin K., Krishnamurthy A., "Simulated responses to support surface disturbances in humanoid biped model with a vestibular like apparatus," IEEE Transactions on Systems, Man and Cybermetics,Vol.40(1), pp.109-119, 2010.

[27] Tian C., He J., "Simulation study of human posture control under external perturbation," IEEE Conference on Decision and Control,San Diego, California, USA.,Vol.3, pp. 2529-2534,1997.

[28] Gollhofer A., Horstmann G.A., Berger W., Dietz V., "Compensation of translational and rotational perturbations in human posture:Stabilization of centre of gravity," Neuroscience Newletters, Vol.105,pp. 73-78.1989.

[29] Winter D.A., "Biomechanics and Motor Control of Human Movement," 4th Edition,John Wiley and Sons Inc.,Hoboken, New Jersy.,2009.

[30] Ishida A., "Responses of the posture-control system to pseudorandom acceleration disturbances," Medical and Biological Engineering Computing, Vol.18(4), pp.433-438,1980.

[31] Pai Y.C., Maki B.E., Iqbal K., Mcllroy W.E., Perry S.D., "Thresholds for step initiation induced by support-surface translation:a dynamic centre of mass model provides much better prediction than static model," Journal of Biomechanics, Vol.33, pp.387-392, 2000.

[32] Maki B.E., Mclllroy W.E., Perry S.D., "Influence of lateral destabilization on compensatory stepping response," Journal of Biomechanics, Vol.29(3), pp.343353,1996 .

[33] Henry S.M., Fung J., Horak F.B., "Control of stance during lateral and anterior and posterior surface translations," IEEE Transactions in Rehabilitation Engineering,Vol.6, pp.32-42,1998. 
[34] Mcllroy W.E., Maki B.E., "The deceleration response to transient perturbation of upright stance," Neurosciene Letters,Vol.175(1-2),pp.13-16,1994.

[35] Brown L.A., Jensen J.L., Korff T., Woollacott M.H., "The translating platform a paradigm: perturbation displacement waveforms alters the postural response," Gait and Posture,Vol.14, pp.256-263,2001.

[36] Graham R., "Motion induced interruptions as ship operatability criteria," Naval Engineers Journal,Vol.102, pp.65-71,1990.

[37] Wedge J., Langlois R.G., "Simulating the effects of ship motion on postural stability using articulated dynamic: models, 2003.

[38] Langlois R.G., MacKinnon S.N., Duncan C.A., "Modelling sea-trial motion induced interruption data using an inverted pendulum articulated postural stability model," International Journal of Maritime Engineering,vol.151,no.a.1,2009.

[39] Ethier C. Ross., Simmons Craig A., "Introductory Biomechanics-From cells to organisms," Cambridge University Press,2007.

[40] Erica N., Sebastiano A., Silvio D. P., Fabio M., Salvatore T., "Low-cost human motion capture system for postural analysis on-board ships," Videometrics, Range Imaging and Applications XI, Proc. Of SPIE, Vol.8085, pp.80850L:180850L:15, 2011.

[41] Winter D.A., "Human balance and posture control during standing and walking," Gait and Posture,Vol.3, pp.193-214,1995.

[42] Patrik K., Hajny O., "Study of human walking by SimMechanics," CTU FBMI Prague, pp:1-10.

[43] Roetenberg D., Luinge H., Slycke P., "Xsens MVN: Full 6DOF Human motion tracking using miniature inertial sensors," XSENS Technologies,pp.1-7, April 8 th,2009.

[44] Centre,"http://www.merriam-webster.com/dictionary/center percent 20of percent 20mass".

[45] Anatomical, "http://en.wikipedia.org/wiki/Anatomical terms of location".

[46] Sungho J., "Hierarchical neural control of human postural balance and bipedal walking in sagittal plane," Ph. D thesis, Massachusetts Institute of Technology,2006. 
[47] Horak F., Nashner L., "Central programming of postural movements:Adaption to altered support surface configurations," Journal of Neurophysiology, Vol.55(6), pp. 1369-1381,1986.

[48] Punakallio A., "Balance abilities of workers in physically demanding jobs: with special reference to firefighters of different ages," National Emergency Training Centre, Vol.4, Supp.8, pp.1-47, 2005.

[49] Stewart D., "A platform with six degree of freedom," Prac. Instt. Mech. Engg,Vol.180, Pt.1, No.15; pp.371-386,1965.

[50] Phillon R.H., Langlois R.G., "Avoiding repetition in ship motion simulation," Proceedings of the Canadian Society for Mechanical Engineering Forum, 01-06, june 2004.

[51] Schulz A., Velho L., "Motion capture: Technical Report," Rio de Janeiro, pp.1$11,2010$.

[52] Randolph A. L., Nelson M., Akkapeddi S., Levin A., Alexandrescu R., "Reliability of measurement of pressures applied on foot during walking by computerized insole sensor system," Arch Phys. Medicine and Rehabilitation, Vol.8, pp.573-578, May 2000.

[53] Fong D. T., Chan Y.Y., Hong Y., Yung P.S., Fung K.Y., Chan K.M., "Estimating the complete ground reaction forces with pressure insoles in walking", Journal of Biomechanics, Vol.41, pp.2597-2601,2008.

[54] Instruction manual, "BB1LP Breath by breath metabolic system", Qubit Systems,pp.1-27.

[55] Palmer G.,Instruction manual, "BB1LP Breath by breath metabolic system", Qubit Systems, pp.1-27.

[56] Robertson E., Gordon D., Caldwell E. G., Hamill J., Kamen G., Whittlesey S. N., "Research Methods in Biomechanics," Human Kinetics,2004.

[57] Wu G., Cavanagh P. R., "ISB Recommendations for standardization in the reporting of kinematic data",Journal of Biomechanics, Vol.28,No.10,pp.12571261,1995 .

[58] Grood E.S., Suntay W.J.,"A joint coordinate system for clinical description of three-dimensional motions: Application to knee",Journal of Biomechanical Engg., Vol.105, pp.136-144,1983. 
[59] Vaughan C.L., Davis B. L., O'Comnor J.C., "Dynamics of human gait", Kiboho Publishers,Second Edition.

[60] Racic V., Pavic A. , Brownjohn J.M.W., "Experimental identification and analytical modelling of human walking forces: Literature review," Journal of Sound and Vibration, Vol.326, pp.1-49,2009.

[61] Rahmtalla S., Xia T., Contrattoc M., Koppc G., Wilderb D., Frey Lawa L., Ankruma J., "Three-dimensional motion capture protocol for seated operator in whole body vibration," International Journal of Industrial Ergonomics, Vol.38, pp.425-433,2008.

[62] Minetti A. E., Ardigo L. P., Reinach E. , Saibene F., "The relationship between mechanical work and energy expenditure of locomotion in horses," The Journal of Experimental Biology, Vol.202, pp.2329-2338,1999.

[63] Donelan J. M., Kram R., Kuo A.D., "Mechanical work for step-to-step transitions is a major determinant of the metabolic cost of human walking," The Journal of Experimental Biology,Vol.205, pp.3717-3727,2002.

[64] Hill A.V., "The heat of shortening and dynamic constants of the muscle," Proceedings of Royal Society Lond. B ,Vol.126, pp.136-195,1938.

[65] Taylor C. R., Heglund N.C., Maloyi G. M. O., "Energetics and mechanics of terresterial locomotion," Journal of Experimental Biology,Vol. 97, pp.97-107,1982.

[66] Curtin N. A., Woledge R. C., "Efficiency of energy conversion during shortening of muscle fibres from the dogfish scyliorhinus canicula," Journal of Experimental Biology, Vol.158, pp.343-353,1991.

[67] Szentesi P., Zaremba R., Van Mechelen W., Stienen G. J. M., "ATP utilization for calcium uptake and force production in different types of human skeletal muscle fibres," Journal of Physiology,Vol.531(pt.2), pp.393-403,2001.

[68] Zarrugh M.Y. ,Todd F.N.,Ralston H.J.,"Optimization of energy expenditure during level walking," European Journal of Applied Physiology,Vol.33, pp.293306,1974 .

[69] I Donelana J.M., Krama R., Kuo A. D., "Simultaneous positive and negative external mechanical work in human walking," Journal of Biomechanics, Vol.35, pp.117-124,2002. 
[70] Deursen R.V., "Mechanical loading and off-loading of the plantar surface of the diabetic foot," Clinical Infectious Diseases ,Vol.39, pp.S87-91, 2004.

[71] Hoang Hoa X., Reinbolt J.A., "Crouched posture maximizes ground reaction forces generated by muscles," Gait and Posture,Vol.36, pp.405-408,2012.

[72] Runge C.F., Shupert C.L., Horak F.B., Zajac F.E.,"Ankle and hip postural strategies defined by joint torques," Gait and Posture,Vol.10, pp.161-170,1999.

[73] Kuo A.D., Zajac F.E., "Human standing posture:Multi-joint movement strategies based on biomedical constraints," Progress in Brain Research,Vol.97, pp.349-358,1999.

[74] Horak F.B., Nashner L.M., "Central programming of postural movements: Adaptation to altered support-surface configurations," "Journal of Neurophysiology,Vol. 55, pp.1369-1381,1986.

[75] White C.R., Seymour R.S., "Mammalian basal metabolic rate is proportional to body mass 2/3," Proc. Natl .Acad. Science, Vol.,100, pp.4046-4049,April 2003.

[76] Ferraro R., Lillioja S., Fontviellie AM, Rising R, Bogardus C, Ravussin E., "Lower sedentary metabolic rate in women as compared to men," J. of Clinical Invest., Vol.90, pp.780-784,1992.

[77] Miller A.E.J., Macdougall J.D., Tarnopolasky M.A., Sale D.G.,"Gender differences in strength and muscle fibre characteristics," European Journal of Applied Physiology, Vol.66, pp.254-262,1993.

[78] Michaelson A.P., Michaelson M., Jaric S., Latash M .L., Sjlander P., Djupsjbacka M.,"Vertical posture and head stability in patients with chronic neck pain," Journal of Rehabilitation Med., Vol.35, pp.229-235,2003.

[79] Wertheim A.H., Heus R, Vrijkotte T.G.M, "Energy expenditure, physical work load and postural control during walking on a moving platform" TNO Defence Resaearch, B-8, pp.1-30,1994.

[80] Wollacott H.M., Tang P. F., "Balance control during walking in the older adult :Research and implications," Physical Therapy, Vol.77, No. 6, pp.646-660,jume 1997.

[81] Cheng K., "A Systematic Perspective of Postural Control," IBBME, pp.1-4. 
[82] Dempster W.T., "Space Requirements of the Seated Operator: Geometrical, Kinematic, and Mechanical Aspects of the Body with Special Reference to the Limbs," WADC Tech. Rept. Wright Air Development Center, Air Research and Development Command, U. S. A. F., Wright-Patterson Air Force Rase, Ohio,pp.55-159,1955.

[83] Cavagna G.A., Franzetti P., "Mechanics of competition walking" Journal of physiology, Vol. 315, pp.243-251,1981.

[84] Willems P.A., Cavagna G.A., Heglund N.C., "External, internal and total work in human locomotion" Journal of experimental biology, Vol.198, pp.379393,1995 .

[85] Donelana J.M., Krama R., Kuo A. D., "Mechanical work for step to step transitions is major determinant of the metabolic cost of human walking," Journal of experimental biology, Vol.205, pp.3717-3727,2002.

[86] Bennett S.W., Lanovaz J.L., Muir G. D., "The biomechanics of locomotor compensation after peripheral nerve lesion in the rat," Behavioural brain research, Vol.229,Issue 2, pp.391-400,2012. 